UNIVERSIDADE DE SÃO PAULO

INSTITUTO DE FÍSICA

\title{
Persistência de ordem em modelos ferromagnéticos na presença de campos auto-similares quase aleatórios
}

\section{Silas Luiz de Carvalho}

Orientador: Prof. Dr. Domingos Humberto Urbano Marchetti

Dissertação de Mestrado
submetida ao Instituto de Física
da Universidade de São Paulo
para a obtenção do título de
Mestre em Ciências.

Banca Examinadora:

Prof. Dr. Sílvio Roberto de Azevedo Salinas (IFUSP)

Prof. Dr. Sacha Friedli (ICEx-UFMG)

Prof. Dr. Domingos Humberto Urbano Marchetti (IFUSP)

São Paulo

2007 



\section{Agradecimentos}

Aos meus pais Nilza Latorre Taranha e Sebastião de Carvalho, aos meus irmãos Silvia, Sergio, Selma e Solange, aos meus avós Edú e Lucrécia, e a minha tia Anameiry, tão importantes para mim.

Ao meu orientador Domingos Humberto Urbano Marchetti, pela excelente orientação.

Aos meus amigos, Rone, Marcelos, Pedro, Thiago, Ivone, José Cláudio, Caio, Andre, Masayuki, Selene, Cristiane, Henrique, Carlos Fiore, Sandro, Danilo, Wellington, Alexei, Gabriel e a muitos outros, que sempre me apoiaram nos momentos bons e difíceis.

A Isis, minha namorada, pelo carinho e pela dedicação.

Às secretárias e demais funcionários do Departamento de Física Geral, pela prestatividade e pela amizade.

Ao meu colega e amigo William Remo Pedroso Conti, por ter gentilmente disponibilizado parte de seu trabalho ao meu uso.

À Fundação de Amparo à Pesquisa do Estado de São Paulo (FAPESP), pelo apoio financeiro. 



\section{Resumo}

Neste trabalho estudamos a existência de ordem de longo alcance em modelos ferromagnéticos na presença de um campo externo cuja configuração apresenta um padrão tipicamente aleatório. Provamos, por meio do argumento de Peierls modificado por Griffiths para o estudo de um antiferromagneto, que o modelo de Ising ferromagnético bidimensional exibe, para um campo alternado de intensidade fraca, ordem de longo alcance à temperatura finita. Propomos dar um passo além considerando campos auto-similares esparsos, cuja soma é nula em todas as escalas. Estudamos também o modelo hierárquico em duas dimensões, para o qual provamos a existência de ordem de longo alcance à temperatura finita, na ausência de campo externo e para um campo com regiões irregulares esparsas. Provamos que os resultados do modelo de contornos hierárquicos são equivalentes aos resultados do modelo hierárquico em duas dimensões. Por fim, provamos através do método do limite infravermelho existência de ordem de longo alcance no modelo N-vetorial com campo alternado, de intensidade fraca, para $d \geq 3$, sob a hipótese de que a variância do estado associado à interação com o campo apresenta cardinalidade inferior a do volume do sistema. Mostramos, sob hipóteses similares, que o modelo N-vetorial hierárquico com campo externo, esparso e de intensidade pequena, apresenta ordem de longo alcance a baixas temperaturas. 



\section{Abstract}

In this work we study the existence of long range order for ferromagnetic models in the presence of an external field whose configuration has a pattern typically random. We prove, via the Peierls' argument modified by Griffiths in his study of an antiferromagnet, that the two dimensional ferromagnetic Ising model for a staggered field exhibits long-range order at finite temperature and small field intensity. We propose to give a further step considering sparse self similar fields, whose sum is zero in all scales. We study as well the hierarchical model in two dimensions, where we prove existence of long-range order at finite temperature in the absence of external field and for a field configuration with sparse irregular regions. We prove that the results for the two-dimensional hierarchical contours model are equivalent to the results of the hierarchical model in two dimensions. Lastly, we prove via infrared bound method, existence of long range order in the $\mathrm{N}$-vector model with a staggered and weak external field for $d \geq 3$, under the hypothesis that the variance of the state connected with the field interaction has cardinality lower than volume. We show, under similar hypotheses, that the N-vector hierarchical model with a sparse field of low intensity has long range ordem at low temperatures. 



\section{Sumário}

1 Introdução 11

2 Modelo de Ising $\quad 18$

2.1 Modelo com campo . . . . . . . . . . . . . . . . . . . . . . . . . . 18

2.2 Argumento de Peierls . . . . . . . . . . . . . . . . . . . . . 21

2.2.1 Prova da existência de magnetização espontânea . . . . . . . 22

2.3 Campo externo quase aleatório . . . . . . . . . . . . . . . . 27

2.4 Aplicação do argumento de Peierls no sistema com campo externo alternado . . . . . . . . . . . . . . . . . . . 28

3 Modelo hierárquico de Dyson 31

3.1 Modelo sem campo . . . . . . . . . . . . . . . . . . . . . . . . 31

3.1.1 Critério para a existência de ordem de longo alcance . . . . 33

3.1.2 Relação de recorrência para o parâmetro de ordem $m^{2} \ldots \ldots \ldots \ldots \ldots$

3.1.3 Teorema principal e desigualdades de Griffiths . . . . . . . . 37

3.1.4 Prova do Teorema 3.1.2 . . . . . . . . . . . . . . . . 40

3.2 Modelo com campo externo quase aleatório . . . . . . . . . . . 45

3.2.1 Na ausência de esparsidade . . . . . . . . . . . . 46

3.2.2 Na presença de esparsidade . . . . . . . . . . . 50

4 Modelo de contornos hierárquicos $\quad 61$

4.1 Procedimento de renormalização . . . . . . . . . . . . . . . . . 61

4.2 Existência de ordem de longo alcance . . . . . . . . . . . . 66 
4.2.1 Resultados para um campo externo não esparso . . . . . . . 66

4.2.2 Resultados para um campo externo esparso . . . . . . . . 71

5 Modelo N-vetorial $\quad 73$

5.1 Modelo com interação entre vizinhos mais próximos .......................... 73

5.1.1 Dominação gaussiana e limite infravermelho para o modelo sem campo externo . . . . . . . . . . . . . 76

5.1.2 Extensão do método do limite infravermelho para o campo alternado ..................... . . 80

5.2 Modelo com interação hierárquica . . . . . . . . . . . . . 86

5.2.1 Laplaciano hierárquico . . . . . . . . . . . . . 87

5.2.2 Ordem de longo alcance na ausência de campo . . . . . . . 93

5.2.3 Na presença de campo quase aleatório . . . . . . . . . . 96

6 Conclusões e problemas em aberto $\quad 104$

$\begin{array}{lr}\text { A Prova do Lema 3.1.4 } & 110\end{array}$

B Dominação gaussiana, positividade por reflexão e estimativa do $\begin{array}{lr}\text { tabuleiro de xadrez } & 114\end{array}$

$\begin{array}{ll}\text { C Positividade por reflexão para o laplaciano hierárquico } & 119\end{array}$ 


\section{Capítulo 1}

\section{Introdução}

O estudo de transições de fase em modelos estatísticos é de fundamental importância, tanto per se como em aplicações. Dentre os modelos estatísticos mais estudados e importantes encontra-se o de Ising, um modelo magnético simples com interação entre primeiros vizinhos que apresenta (na ausência de campo magnético) em $d \geq 2$ uma transição de fase, de uma fase ordenada a baixas temperaturas para uma fase desordenada a altas temperaturas.

Para sistemas com simetria discreta, como o modelo de Ising, há um método de grande importância para estabelecer a existência de uma transição de fase: o argumento de Peierls. Ele não requer invariância translacional do modelo, mas infelizmente não há versões do argumento para sistemas com simetrias contínuas, um dos grandes problemas abertos em mecânica estatística e teorias de campos. Griffiths [12] tornou o argumento rigoroso, e Pirogov-Sinai [21] desenvolveram uma teoria, baseada nas estimativas de Peierls, para descrever diagramas de fase de baixas temperaturas de sistemas clássicos.

A presença de um campo externo uniforme destrói a ordem do sistema ferromagnético em qualquer dimensão e temperatura. A ordem de longo alcance é, no entanto, preservada na presença de um campo externo alternado e de um campo aleatório caso a intensidade de ambos seja suficientemente fraca.

O modelo de Ising ferromagnético com campo aleatório (MIFCA) é de grande interesse físico, uma vez que pode ser empregado na descrição de inúmeros sistemas; podemos citar como exemplos: antiferromagnetos diluídos altamente anisotrópicos 
em um campo externo homogêneo; a estatística de gotículas de um fluido (por exemplo óleo) em um meio poroso; ondas de densidade de carga comensuráveis em sólidos contendo impurezas, distribuídas aleatoriamente.

A estimativa de Peierls foi empregada por Imry e Ma [14] em sua argumentação sobre a dimensão crítica inferior $d_{\text {inf }}$ do MIFCA. Segundo estes autores, o estado de um sistema é determinado pela competição entre o campo aleatório, cujo efeito cumulativo em uma região hipercúbica $\Lambda=[-L, L]^{d}$ é de ordem $\sqrt{|\Lambda|}$, e o mecanismo de quebra de simetria, cuja intensidade é da ordem da fronteira $|\partial \Lambda|=L^{d-1}$. Para o caso marginal de $d=2$, a predição é de que o campo flutuante apresentará efeito dominante. O argumento de Imry e Ma foi confirmado rigorosamente por Imbrie [13] e Bricmont-Kupiainen [2], que demostraram magnetização espontânea no MICA para desordem fraca e $d \geq 3$, se $T=0$ e $T \geq 0$ suficientemente pequeno, respectivamente.

O presente trabalho consiste na utilização de estimativas de Peierls para investigar a persistência de ordem no modelo de Ising ferromagnético bidimensional na presença de um campo magnético, de intensidade fraca, cuja configuração é construída pela repetição de um padrão tipicamente aleatório, em todas as escalas (com uma estrutura hierárquica). O campo empregado em nosso estudo, denominado quase aleatório, é apresentado e discutido em detalhes na Seção 2.3.

De acordo com um teorema de Aizenman e Wehr (vide [1]), o MIFCA em dimensão $d=2$ não apresenta transição de primeira ordem com respeito ao parâmetro de ordem associado ao campo desordenado pois, devido a um efeito de arredondamento, a magnetização não pode ser descontínua. Conseqüentemente, se o campo for escolhido segundo uma distribuição independente para cada sítio, não há magnetização espontânea. O teorema, no entanto, não se aplica ao problema em questão, já que o campo não é estacionário com respeito a translações (exigência do teorema), como veremos, e além disso é fortemente correlacionado.

Diferentemente das propostas anteriores que utilizam a lei dos grandes números e o teorema central do limite para estimar flutuações do campo efetivo, objetivamos entender como lidar com configurações específicas do campo magnético. Esta mudança de ponto de vista levou a simplificações importantes em outros problemas 
envolvendo desordem, o que justifica uma tentativa nessa direção.

Buscamos ainda uma maior compreensão do mecanismo de transição de fase na presença do campo aleatório. Enquanto que o argumento de Imry-Ma não é rigoroso e é incompleto no sentido de não tratar o efeito de contornos dentro de contornos (que tende a reduzir as flutuações do campo graças a um efeito de "blindagem"), os resultados atribuídos a Imbrie $(T=0)$ e a Bricmont-Kupiainen $(T>0)$ são deveras técnicos e pouco contribuem para o entendimento da natureza física do problema. Desse modo, a escolha de um campo determinístico com padrão aleatório é mais uma vez justificada.

Há dois motivos para a estudarmos o caso $d=2$. Excluíndo os contornos internos na representação de Peierls do MIFCA, Pontin, Baêta e Perez [22] demostraram a existência de uma transição de fase. A outra razão do interesse no modelo bidimensional é que o teorema de Aizenman-Wehr não resolve a questão da existência de alguma transição de fase no MIFCA em duas dimensões.

Ainda tendo em mente o estudo da persistência da fase ferromagnética no modelo de Ising com campo, propomos o estudo do modelo hierárquico (MHD), introduzido por Dyson em [4]. Neste trabalho, Dyson mostra a existência de uma fase ordenada de baixa temperatura para sistemas ferromagnéticos com interação de decaimento lento a partir da construção de uma relação de recorrência para o quadrado da magnetização espontânea (parâmetro de ordem do modelo com condições de fronteira periódicas ou livres). O MHD substitui a distância euclideana, presente na interação ferromagnética de dois corpos dos modelos usuais, pela chamada distância hierárquica, que separa os sítios da rede em blocos (hierárquicos).

Além de apresentar cálculos mais explícitos e diretos, o que torna seu estudo interessante per se, a existência de ordem de longo alcance (associada no caso à existência de uma fase ferromagnética a baixas temperaturas) para o MHD bidimensional com campo garante, via desigualdades de Griffiths, existência de ordem no modelo de Ising ferromagnético bidimensional com campo. Isto porque as desigualdades nos dizem que um fortalecimento das ligações ferromagnéticas de um modelo levam a um aumento das correlações entre pontos distintos, ou seja, a um aumento na ordenamento dos spins (o MHD apresenta interações ferromagnéticas 
mais fracas que as do modelo de Ising). Outro motivo para estudarmos o MHD é a configuração hierárquica do campo magnético, que busca simular uma configuração tipicamente aleatória, caracterizado pela repetição de padrões em múltiplas escalas.

Propomos também o estudo do chamado modelo de contornos hierárquicos $(\mathrm{MCH})$, investigado por Bricmont e Kupiainen em [3], como um complemento ao nosso estudo de existência de ordem de longo alcance, buscando reforçar os resultados obtidos. Os contornos são fronteiras dos hipercubos (quadrados em $d=2$ ) identificados com os blocos hierárquicos. O método envolve um processo de renormalização exato que nos permite obter um relação de recorrência para a magnetização (parâmetro de ordem para sistemas com condições de fronteira não neutras).

Por fim, propomos o estudo de ordem de longo alcance em sistemas com simetria contínua na presença de campo magnético. Sistemas com quebra de simetria contínua apresentam diversas peculiaridades interessantes. Em primeiro lugar, não há quebra de simetria contínua para $d \leq 2$ (em contraste com o caso discreto, que possui essa quebra em $d=2$ ). Este é o teorema de Goldstone-Mermin-Wagner. Em segundo lugar, a existência de ordem de longo alcance para $d \geq 3$ é de natureza de uma "condensação de ondas de spin", analisada pelo método do limite infravermelho (proposto por Fröhlich, Simon e Spencer em [9]). A idéia do método consiste em substituir as integrais que surgem nos cálculos (como exemplo a magnetização espontânea) por integrais gaussianas, solúveis.

Especificamente, estudamos os modelos N-vetoriais usual e hierárquico (neste último empregamos o laplaciano hierárquico), em que o limite infravermelho decorre da chamada "dominação gaussiana". Nestes modelos, o spin é um vetor de N componentes na esfera unitária do espaço euclideano de $\mathrm{N}$ dimensões. O resultado de dominação gaussiana envolve a chamada positividade por reflexão da medida de Gibbs associada ao modelo. Utilizamos o mesmo campo magnético empregado no estudo dos modelos com simetria discreta, com padrão aleatório.

Nossa contribuição para a literatura se encontra na prova da existência de magnetização positiva para os seguintes modelos: o modelo de Ising ferromagnético com campo alternado (via argumento de Peierls; isto segue também da existência de ordem no anti-ferromagneto com campo uniforme estabelecida por Griffiths [11]; 
vide Seção 2.4); o modelo hierárquico de Dyson em duas dimensões na presença de campo externo com blocos irregulares esparsos (Seção 3.2; vide Seção 2.3 para a definição de bloco irregular); o modelo de contornos hierárquicos, também na presença de campo externo com blocos irregulares esparsos (Seção 4.2); o modelo N-vetorial com campo externo alternado (sob a Hipótese 5.2.2; vide Subseção 5.1.2); o modelo N-vetorial hierárquico, na ausência de campo (Subseção 5.2.2), na presença de um campo alternado e de um campo com blocos irregulares super-exponencialmente esparsos (Subseção 5.2.3).

Por conveniência, incluimos no início de cada capítulo seções preliminares contendo técnicas, métodos e resultados empregados nesta dissertação. Extraímos o Argumento de Peierls, apresentado na Seção 2.2, de [11]. A prova da existência de ordem de longo alcance para o modelo de Dyson na ausência de campo, presente na Seção 3.1, foi literalmente extraída das notas de aula [17] e a Proposição 4.1.1, apresentada na Seção 4.1, de [3]. A existência de magnetização positiva para o modelo N-vetorial sem campo, presente na Subseção 5.1.1, baseia-se em [19]. Por fim, a prova do Teorema Espectral para o laplaciano hierárquico (Teorema 5.2.1), bem como a Subseção 5.2.1, foram literalmente extraídas de [18].

A dissertação está assim dividida:

No Capítulo 1, definimos o modelo de Ising ferromagnético bidimensional com campo magnético externo (inclusive aleatório) e expomos o argumento de Peierls original. Em seguida, definimos a configuração do campo externo a ser estudada e por fim buscamos verificar a persistência do argumento mediante a presença deste campo no sistema.

No Capítulo 2, apresentamos a generalização do argumento usado por Dyson para demonstrar a existência de ordem de longo alcance no MHD com geometria bidimensional. Em primeiro lugar, analisamos o modelo na ausência de campo, onde adquirimos intuição para tratar o problema com campo, introduzido logo em seguida. Buscamos assim uma resposta para a existência da fase ordenada de baixa temperatura. Os resultados expressivos constituem o Teorema 3.1.2, relacionado à existência de ordem de longo alcance à temperatura finita, e a sua generalização para o modelo com os blocos irregulares do campo esparsos em uma escala que 
cresce exponencialmente rápido (vide Subseção 3.2.2).

No Capítulo 3, introduzimos o MCH. Em seguida, definimos o processo de renormalização empregado no cálculo da função de partição do modelo, bem como da magnetização (função de um ponto) na presença de campo externo, visando provar a existência de uma fase ordenada para baixas temperaturas (consideramos o limite assintótico $\beta \rightarrow \infty)$. Provamos, para o mesmo campo com blocos irregulares esparsos em uma escala de crescimento exponencial, a existência de tal fase.

No Capítulo 4, definimos o modelo N-vetorial com e sem campo externo e o método do limite infravermelho, empregado no estudo da existência de ordem de longo alcance. Constatamos a existência desta ordem para um campo alternado de intensidade fraca, em $d \geq 3$, sob a suposição de que a variância do modo alternado (que também exibe uma ocupação macroscópica) possua cardinalidade inferior a do volume. Em seguida, expomos o modelo N-vetorial hierárquico, definido a partir do laplaciano hierárquico, e então repetimos os passos empregados no estudo do modelo usual. Verificamos que para um campo quase aleatório, com regiões irregulares esparsas em uma escala com crescimento super-exponencial (controlado por um parâmetro $\alpha$ ), o sistema exibe ordem de longo alcance a baixas temperaturas e a campo fraco se $d>\max \left(2 \alpha, \frac{4 \alpha}{\alpha-1}\right)$ (vide Proposição 5.2.3). Já o modelo sem campo exibe a mesma ordem para $d \geq 3$.

No Capítulo 5, encontram-se as conclusões que extraímos dos resultados obtidos e discutidos no decorrer dos capítulos anteriores, bem como alguns problemas que permanecem em aberto. Podemos resumir as conclusões como: os sistemas bidimensionais com simetria discreta (Ising, MHD e MCH) apresentam ordem de longo alcance para campos alternados (neutros em todas as escalas), enquanto que os dois últimos apresentam esta ordem na situação de um campo com blocos irregulares esparsos, de crescimento exponencial (sem esparsidade, os blocos irregulares criam flutuações suficientes para criar domínios e destruir a ordem de longo alcance); o modelo N-vetorial é um pouco distinto, uma vez que a ocupação macroscópica de infinitos estados associados à interação com o campo destrói a ordem de longo alcance, a menos que façamos as regiões irregulares esparsas em uma escala com crescimento super-exponencial. 
Por fim, o Apêndice A exibe a prova do Lema 3.1.4, uma generalização do lema presente em [4] para $d \geq 2$ e tamanho do bloco $L \geq 2$; nos Apêndices B e C apresentamos o conceito de positividade por reflexão para os laplacianos usual e hierárquico, respectivamente, e seu papel na dominação gaussiana. 


\section{Capítulo 2}

\section{Modelo de Ising}

Pretendemos utilizar estimativas de Peierls para demonstrar a persistência da fase ordenada do modelo de Ising na presença de um campo $h: x \in \mathbb{Z}^{2} \rightarrow h_{x} \in\{1,-1\}$, de intensidade fraca, controlada por um parâmetro $\epsilon$, cuja configuração consiste na repetição de um padrão tipicamente aleatório em todas as escalas. Na Seção 2.1 apresentamos o modelo e alguns resultados associados ao modelo com campo aleatório; na Seção 2.2 expomos o argumento de Peierls; na Seção 2.3 definimos o campo quase aleatório; finalmente na Seção 2.4 exibimos o resultado da aplicação do argumento sobre o sistema com o campo alternado; não foi possível extrair nenhum resultado para o modelo com campo quase aleatório a partir deste método.

\subsection{Modelo com campo}

O modelo de Ising ferromagnético com campo é um sistema de spins em $\mathbb{Z}^{d}$ cujos estados de equilíbrio são definidos pela medida estacionária de Gibbs.

Uma configuração $\sigma$ é um elemento do espaço produto $\mathbf{Y}=\{-1,1\}^{\mathbb{Z}^{d}}$. Quando o modelo é definido em $\Lambda \subset \mathbb{Z}^{d}$, o conjunto configuracional é $\mathbf{Y}_{\Lambda}=\{-1,1\}^{\Lambda}$.

Seja $V$ um hipercubo centrado na origem; façamos $\Lambda=V \cap \mathbb{Z}^{d}$. Uma condição de fronteira para $\Lambda$ é dita especificada se $\eta \in \mathbf{Y}$ é escolhida. Qualquer configuração $\sigma^{\Lambda} \in \mathbf{Y}_{\Lambda}$ se estende para uma configuração $\sigma \in \mathbf{Y}$ como 


$$
\sigma_{j}=\left\{\begin{array}{ccc}
\sigma_{j}^{\Lambda} & \text { se } & j \in \Lambda \\
\eta_{j} & \text { se } & j \notin \Lambda
\end{array} .\right.
$$

Uma condição de contorno positiva é escolhida se $\eta \equiv 1\left(\eta_{j}=1, j \in \mathbb{Z}^{d}\right)$. Analogamente, $\eta \equiv-1$ define uma condição de contorno negativa.

Para cada realização do campo $h$ e para cada configuração de spin, com condição de fronteira $\eta$, associamos uma energia

$$
H_{\Lambda}^{\eta}(\sigma, h)=H_{\Lambda}(\sigma, h)+B_{\Lambda}^{\eta}(\sigma)
$$

em que

$$
H_{\Lambda}(\sigma, h)=-\frac{1}{2} \sum_{\substack{|i-j|=1, i, j \in \Lambda}} \sigma_{i} \sigma_{j}-\epsilon \sum_{i \in \Lambda} h_{i} \sigma_{i}
$$

é a energia do sistema sem interação com a fronteira $(|\cdot|$ é a norma euclideana e $|i-j|=1$ representa vizinhos mais próximos na rede) e

$$
B_{\Lambda}^{\eta}(\sigma)=-\frac{1}{2} \sum_{\substack{|i-j|=1, i \in \Lambda, j \notin \Lambda}} \sigma_{i} \eta_{j}
$$

é a interação devida à condição de fronteira $\eta$.

O campo $h$ é dito ser alternado se for positivo na sub-rede par e negativo na sub-rede ímpar:

$$
h_{x}=\left\{\begin{array}{rll}
1 & \text { se } & x \in \Lambda_{\mathrm{p}} \\
-1 & \text { se } & x \in \Lambda_{\mathrm{i}}
\end{array},\right.
$$

$\operatorname{com} \Lambda_{\mathrm{p}(\mathrm{i})} \equiv\left\{x \in \Lambda \cap \mathbb{Z}^{d}: x_{1}+\cdots+x_{d}=\operatorname{par}(\right.$ ímpar $\left.)\right\}$. Usualmente, $h=\left\{h_{x}\right\}_{x \in \mathbb{Z}^{d}}$ é uma coleção de variáveis aleatórias independentes e identicamente distribuídas (v.a.i.i.d.) de média zero e variância um. O parâmetro $\epsilon$ está associado à intensidade da desordem (intensidade do campo). 
O valor esperado de uma função $g: \Lambda \rightarrow \mathbb{R}$ com respeito à medida de Gibbs

$$
\mu_{\Lambda}^{\eta}(\sigma, h):=\frac{1}{Z_{\Lambda}^{\eta}(h)} e^{-\beta H_{\Lambda}^{\eta}(\sigma, h)}
$$

é definido como

$$
\langle g\rangle_{\Lambda}^{\eta}(h)=\frac{1}{Z_{\Lambda}^{\eta}(h)} \sum_{\sigma} g(\sigma) e^{-\beta H_{\Lambda}^{\eta}(\sigma, h)}
$$

em que

$$
Z_{\Lambda}^{\eta}(h)=\sum_{\sigma} e^{-\beta H_{\Lambda}^{\eta}(\sigma, h)}
$$

é a função de partição e $\beta$ é o inverso da temperatura.

Denotamos por $\langle\cdot\rangle^{\eta}=\lim _{\Lambda \rightarrow \mathbb{Z}^{d}}\langle\cdot\rangle_{\Lambda}^{\eta}$ o limite termodinâmico da medida $\langle\cdot\rangle_{\Lambda}^{\eta}$. No caso $h$ v.a.i.i.d., distinguimos três regimes:

1. No regime desordenado $(\epsilon \gg 1)$, a função de correlação decai

$$
\left\langle\sigma_{x} \sigma_{y}\right\rangle^{\eta}-\left\langle\sigma_{x}\right\rangle^{\eta}\left\langle\sigma_{y}\right\rangle^{\eta} \underset{|x-y| \rightarrow \infty}{\longrightarrow} 0
$$

com probabilidade um, exponencialmente rápido, independentemente de $\eta$ (vide [8] para a prova). Esta propriedade é denominada "mixing".

2. Se $\epsilon \ll 1$ e $d>2$, vale a desigualdade

$$
\left\langle 1-\sigma_{0}\right\rangle_{\Lambda}^{+} \leq e^{-c \beta}
$$

uniformemente em $\Lambda$, com probabilidade pelo menos $1-e^{-c^{\prime} / \epsilon^{2}}$ e os estados $\langle\cdot\rangle^{ \pm}$satisfazendo (2.1.4) com probabilidade um (vide [2]). Este fenômeno é denominado quebra espontânea de simetria. 
3. Se $\epsilon \ll 1$ e $d=2$, não há quebra espontânea de simetria (vide [1]) e é ainda uma questão não respondida se existe alguma transição de fase.

Na próxima seção apresentamos o argumento de Peierls, empregado na prova da existência de magnetização espontânea no modelo de Ising ferromagnético bidimensional sem campo magnético.

\subsection{Argumento de Peierls}

Partimos da definição de energia livre $f_{\Lambda}$ para um conjunto finito de sítios $\Lambda$ (com condições de fronteira livres):

$$
f_{\Lambda}(h):=-\frac{1}{\beta|\Lambda|} \log Z_{\Lambda}(h)
$$

Consideramos aqui um campo magnético $h$ uniforme, isto é, $h_{x}=h \forall x \in \mathbb{Z}^{d}$. A energia livre é uma função côncava de $h$ (vide [11]), bem como simétrica,

$$
f_{\Lambda}(h)=f_{\Lambda}(-h)
$$

fixado o inverso da temperatura $\beta$. Ambas propriedades são herdadas pelo limite termodinâmico $f \equiv \lim _{\Lambda \uparrow \mathbb{Z}^{d}} f_{\Lambda}$ quando este existe. A magnetização por sítio

$$
M=-\frac{\partial f}{\partial h}
$$

é uma função monótona, não-decrescente em $h$ e anti-simétrica

$$
M(-h)=-M(h)
$$

Observe que a concavidade de $f$ em $h$ implica que a derivada (2.2.6) é bem definida exceto, no máximo, em um número contável de descontinuidades. Em particular, o limite para $h \geq 0$ 


$$
M_{0}=\lim _{h \rightarrow 0^{+}} M(h)
$$

sempre existe e nunca é negativo. Se $M_{0}>0$, fica claro de $(2.2 .7)$ que $M(h)$ é descontínua na origem. Dizemos neste caso que o sistema apresenta uma magnetização espontânea.

\subsubsection{Prova da existência de magnetização espontânea}

A prova de que o modelo de Ising ferromagnético apresenta transição de fase em $h=0$ para temperaturas suficientemente pequenas se conduz em duas etapas:

1. Mostramos que para condições positivas de fronteira, a magnetização por sítio $M_{\Lambda}^{+}$para um sistema finito

$$
M_{\Lambda}^{+}=-\frac{\partial f_{\Lambda}^{+}}{\partial h}=\frac{1}{|\Lambda|} \sum_{i \in \Lambda}\left\langle\sigma_{i}\right\rangle
$$

possui em $h=0$ um limitante inferior

$$
M_{\Lambda}^{+} \geq \alpha>0,
$$

com $\alpha$ independente de $\Lambda$.

2. Mostramos que $\alpha$ é também um limitante inferior para $M_{0}$, definido por (2.2.8).

Consideremos o sistema finito $\Lambda$ como sendo um quadrado (é suficiente tratar o caso $d=2$; a extrapolação para mais dimensões segue como feito até então) com condições positivas de fronteira (para o caso em que as condições de fronteira são arbitrárias vide [12]).

Quando escolhemos condições positivas de fronteira para $\Lambda$, podemos estabelecer uma correspondência biunívoca entre uma configuração $\sigma$ e um conjunto $\{\gamma\}$ de 
contornos fechados que não se interceptam da seguinte forma. Sejam $\Lambda_{-}=\Lambda_{-}(\sigma)$ tal que $\sigma_{j}=-1$ para $j \in \Lambda_{-}$e $\sigma_{j}=1$ para $j \in \mathbb{Z}^{d} \backslash \Lambda_{-}$e $\Gamma_{\Lambda}$ o conjunto das componentes conexas (e disjuntas) da fronteira $\partial \Lambda_{-}$(um subconjunto $A \subset \mathbb{Z}^{d}$ é dito conexo se para quaisquer dois pontos $t, t^{\prime} \in A$, existe um caminho com ponto inicial $t$, ponto final $t^{\prime}$ e passos entre sítios vizinhos de $A$ ). Definimos os contornos $\gamma$ como elementos de $\Gamma_{\Lambda}$.

Um contorno de comprimento (perímetro) $b$ consiste de $b$ segmentos retos e envolve no máximo $(b / 4)^{2}$ sítios da rede (desigualdade isoperimétrica).

Suponha que haja $\nu(b)$ formas possíveis de desenhar um contorno de comprimento $b$ em $\Lambda$. Seja $X_{b}^{j}$ a seguinte função indicadora:

$$
X_{b}^{j}=\left\{\begin{array}{lll}
1 & \text { se } & \gamma_{b}^{j} \in \mathbf{X} \\
0 & \text { se } & \gamma_{b}^{j} \notin \mathbf{X}
\end{array},\right.
$$

em que $\gamma_{b}^{j}$ corresponde ao $j$-ésimo contorno de comprimento $b$ e $\mathbf{X}$ é a coleção de todos os possíveis contornos de uma dada configuração.

Para qualquer configuração satisfazendo as condições de fronteira mencionadas, o número médio de sítios $\Lambda_{-}$(em que $\left.\sigma_{i}=-1\right)$ é limitado por

$$
\left\langle\Lambda_{-}\right\rangle \leq \sum_{b=4,6 \ldots}(b / 4)^{2} \sum_{j=1}^{\nu(b)}\left\langle X_{b}^{j}\right\rangle .
$$

Uma vez que

$$
M_{\Lambda}^{+}=\frac{\left\langle\Lambda_{+}\right\rangle-\left\langle\Lambda_{-}\right\rangle}{|\Lambda|}=1-2 \frac{\left\langle\Lambda_{-}\right\rangle}{|\Lambda|}
$$

devemos apenas mostrar que o membro direito de (2.2.11) não excede a quantidade $(1-\alpha)|\Lambda| / 2$, pois assim

$$
M_{\Lambda}^{+} \geq 1-2 \frac{(1-\alpha)|\Lambda|}{2|\Lambda|}=\alpha
$$

Aqui surge o argumento chave. Um limitante superior de $\left\langle X_{b}^{j}\right\rangle$ para um contorno específico de comprimento $b$ pode ser construído usando-se a definição 


$$
\left\langle X_{b}^{j}\right\rangle=\frac{\sum_{\sigma}^{\prime} e^{-\beta H_{\Lambda}(\sigma)}}{\sum_{\sigma} e^{-\beta H_{\Lambda}(\sigma)}},
$$

sendo o numerador uma soma sobre todas as configurações em que o contorno se faz presente e o denominador uma soma irrestrita sobre todas as configurações.

Para cada configuração $C$ em que o contorno aparece associamos uma configuração $C^{*}$, obtida pela troca $\left(\sigma_{i} \rightarrow-\sigma_{i}\right)$ de todos os spins no interior do contorno. Suas energias são relacionadas por (note que $h=0$ )

$$
H\left(C^{*}\right)=H(C)-2 b,
$$

uma vez que a configuração $C^{*}$ elimina o contorno $\gamma_{b}^{j}$, e este por sua vez tem um peso energético proporcional a seu comprimento (mais precisamente proporcional ao número de ligações que o constituem).

Um limitante superior de (2.2.12) é obtido excluíndo do denominador todas as configurações exceto às configurações $C^{*}$ correspondentes ao contorno $\gamma_{b}^{j}$ (note que o somando é positivo):

$$
\left\langle X_{b}^{j}\right\rangle \leq \exp \{-2 \beta b\}
$$

Um limitante superior para $\nu(b)$ é obtido notando que temos no máximo $|\Lambda|$ contornos de um dado tipo (dois contornos são do mesmo tipo se um pode ser obtido do outro a partir de uma translação rígida). Um contorno de um dado tipo pode ser construído seqüencialmente ligando um segmento por vez àqueles já presentes. Em cada estágio desse processo (a posição do primeiro segmento é arbitrária) há no máximo 3 possibilidades de escolha do próximo segmento; há, portanto, um máximo de $4 \cdot 3^{b-1}$ tipos de contornos. Temos então que

$$
\nu(b) \leq 4|\Lambda| 3^{b-1}
$$

Inserindo (2.2.13) e (2.2.14) em (2.2.11), obtemos 


$$
\frac{\left\langle\Lambda_{-}\right\rangle}{|\Lambda|} \leq \frac{1}{|\Lambda|} \sum_{b=4,6 \ldots}(b / 4)^{2} \sum_{j=1}^{\nu(b)} e^{-2 \beta b}=\frac{1}{12} \sum_{b=4,6 \ldots} b^{2}\left(3 e^{-2 \beta}\right)^{b} .
$$

É evidente que pela simples escolha de $\beta$ suficientemente grande (isto é, a temperatura suficientemente baixa), o membro direito de (2.2.15) pode ser feito tão pequeno quanto se queira, em particular menor que $(1-\alpha) / 2$, independentemente de $\Lambda$.

Este é o ponto crucial de nossa análise: a estabilidade do argumento de Peierls ao introduzirmos uma perturbação (no caso o campo magnético) está diretamente ligada à convergência da soma em (2.2.15). Podemos reescrevê-la como

$$
\frac{\left\langle\Lambda_{-}\right\rangle}{|\Lambda|}(h) \leq \sum_{\gamma: \gamma \in \Gamma_{\Lambda}} e^{\beta F_{V(\gamma)}(h)} e^{-2 \beta|\gamma|}
$$

com

$$
\beta F_{V(\gamma)}(h) \equiv \log \left(\frac{Z_{V(\gamma)}^{-}}{Z_{V(\gamma)}^{+}}\right)
$$

como em (2.2.5). Aqui $Z_{V(\gamma)}^{ \pm}$é a função de partição no volume $V(\gamma)$ com condições internas de fronteira $\pm ; V(\gamma)$ é o volume determinado pelo contorno $\gamma$ e como sempre $|\gamma|$ indica sua cardinalidade.

A convergência da soma em (2.2.16) depende da razão $Z_{V(\gamma)}^{-} / Z_{V(\gamma)}^{+}$; caso a quantidade $\log \left(Z_{V(\gamma)}^{-} / Z_{V(\gamma)}^{+}\right)$contribuia com um termo proporcional à cardinalidade do contorno $\gamma$, então o argumento persiste; caso contrário este deixa de ser válido. Vale notar que essa condição deve ser satisfeita para todos os contornos simultaneamente; caso um a viole o argumento deixa de persistir.

Ou seja, deve valer

$$
e^{\beta F_{V(\gamma)}(h)} e^{-2 \beta|\gamma|} \leq e^{-m|\gamma|}
$$


para algum $m=m(\beta)$ independente de $\gamma$ tal que $m(\beta) \rightarrow \infty$ quando $\beta \rightarrow \infty$. Note que tal razão é uma generalização de (2.2.12) quando se faz presente o campo magnético.

A energia livre $F_{V(\gamma)}(h)$ induzida por $\gamma$ satisfaz as seguintes propriedades:

1. Se $\epsilon=0$ então

$$
F_{V(\gamma)}(h)=0 \text {. }
$$

2. Se $\epsilon>0, F_{V(\gamma)}(h)$ é uma variável anti-simétrica com respeito a $h \rightarrow-h$, uma vez que $Z_{V}^{+}(h)=Z_{V}^{-}(-h)$.

3. Restringindo as condições de tal forma que cada contorno $\gamma$ não contenha contornos internos, temos

$$
F_{V(\gamma)}(h)=2 \sum_{i \in V(\gamma)} h_{i}
$$

Por fim, a segunda parte do argumento segue da desigualdade

$$
f_{\Lambda}^{+}(h)-f_{\Lambda}^{+}(0) \leq h f_{\Lambda}^{\prime+}(h)=-h M_{\Lambda}^{+}(h),
$$

uma vez que $f_{\Lambda}^{+}$é uma função côncava de $h$. Sendo $M_{\Lambda}^{+}$monótona crescente, (2.2.9) implica que

$$
f_{\Lambda}^{+}(h)-f_{\Lambda}^{+}(0) \leq-h \alpha
$$

para $h \geq 0$. No entanto, à medida que $\Lambda \uparrow \mathbb{Z}^{2}, f_{\Lambda}^{+}(h)$ converge para o mesmo limite $f(h)$, uma vez que as condições de fronteira contribuem apenas com um termo de superfície para a energia livre (vide [11]). Dessa forma $f(h)$ também satisfaz (2.2.18) para $h \geq 0$, o que significa que $M_{0}$ não pode ser menor que $\alpha$ :

$$
\lim _{h \rightarrow 0^{+}}\left(-\frac{f(h)-f(0)}{h}\right)=M_{0} \geq \alpha .
$$

Isto encerra a prova. 


\subsection{Campo externo quase aleatório}

Apresentamos nesta seção a configuração para o campo externo que utilizamos em nossa investigação. A idéia é adotar uma configuração que apresente estrutura multi-escalar (repetição de um dado padrão em todas as escalas), seja suficientemente regular e simule um padrão aleatório. Em vista destas características, denominamos esse campo de quase aleatório. Com essa dada escolha tentamos obter mais facilmente resultados e identificar os pontos principais para o entendimento do problema estudado.

As escalas são, por exemplo, definidas pela seqüência monótona crescente $\mathcal{L}=\left\{L_{n}\right\}_{n \in \mathbb{N}} \operatorname{com} L_{n}=L^{n}$. Adotamos aqui $L=2$. A configuração é construída indutivamente por intermédio do conceito de admissibilidade.

Um bloco configuracional pertencente à primeira escala é dito admissível com respeito à primeira escala (1-admissível) se o campo externo for alternado, isto é, dado $i \in \Lambda_{1}$ todos os seus primeiros vizinhos apresentarão o campo com mesma intensidade, porém com sinal oposto ao seu (vide (2.1.2)). Um bloco $\Lambda_{1}$ é dito 1-irregular se o campo externo que o constitui for inteiramente alinhado, isto é, caso este apresente o mesmo sinal em toda parte. Vemos desse modo a possibilidade de construir dois tipos de blocos 1-irregulares; os blocos $(1,+)$-irregulares, cujo campo apresenta sinal positivo, e os blocos $(1,-)$-irregulares, cujo campo é negativo. Como nos blocos 1-admissíveis, a intensidade do campo é a mesma em todos os pontos dos blocos 1-irregulares.

Passemos para a segunda escala. Definimos um bloco $\Lambda_{2}$ 2-admissível como aquele constituído quase inteiramente pela união de blocos 1-admissíveis (dois para $L=2)$, exceto por um bloco $(1,+)$-irregular e um bloco $(1,-)$-irregular. Definimos blocos $(2,+)$-irregular e $(2,-)$-irregular como a união de $L^{2}$ blocos $(1,+)$-irregulares e $(1,-)$-irregulares, respectivamente. Note que a soma dos valores do campo em um bloco 2-admissível é nula, assim como o de um bloco 1-admissível.

Indutivamente, dizemos que um bloco $\Lambda_{n}$ é $n$-admissível caso este seja constituído pela união de blocos $(n-1)$-admissíveis (dois para $L=2$ ), exceto pela presença de um bloco $(n-1,+)$-irregular e um bloco $(n-1,-)$-irregular. Já os blocos $(n,+)$-irregular e $(n,-)$-irregular são definidos como a união de blocos 
$(n-1,+)$-irregulares e $(n-1,-)$-irregulares respectivamente.

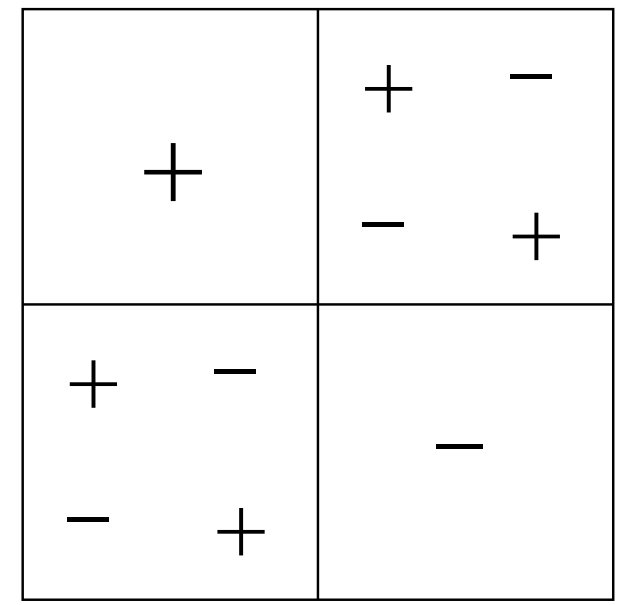

Figura 2.1: Representação esquemática de um bloco 2-admissível para uma escala $L_{n}=L^{n}, \operatorname{com} L=2$.

\subsection{Aplicação do argumento de Peierls no sistema com campo externo alternado}

Iniciemos nosso estudo através da utilização de um campo externo do tipo alternado. Novamente buscamos verificar as condições segundo as quais ocorre magnetização espontânea. Como visto pelo argumento de Peierls (Seção 2.2), a existência de magnetização espontânea depende do comportamento das razões entre as funções de partição internas aos contornos: caso a contribuição associada a cada uma destas seja dominada pelo peso energético do contorno o argumento resiste. Para a situação que propomos estudar devemos usar um argumento de Peierls ligeiramente modificado (análoga à prova para o modelo antiferromagnético com campo uniforme desenvolvida em [11]). Consideramos um sistema com condições positivas de fronteira.

Seja $X_{b}^{j}$ a função indicadora do j-ésimo contorno de comprimento $b$ que surge em uma dada configuração, como em (2.2.10). Consideremos mais uma vez a média 


$$
\left\langle X_{b}^{j}\right\rangle=\frac{\sum_{\sigma}^{\prime} e^{-\beta H_{\Lambda}(\sigma)}}{\sum_{\sigma} e^{-\beta H_{\Lambda}(\sigma)}} .
$$

Obtenhamos um limitante superior para $\left\langle X_{b}^{j}\right\rangle$. Em vez de simplesmente trocarmos o sinal dos spins no interior do contorno, adotamos o seguinte procedimento.

Seja $C$ uma configuração na qual o contorno $\gamma_{j}$ ocorre, $\sigma_{k}$ e $\sigma_{k}^{*}$ os valores dos spins no sítio $k$ das configurações $C$ e $C^{*}$ respectivamente. Definimos a configuração $C^{*}$ como segue: se $k$ é um sítio pertencente à rede $\Lambda$, seja $\hat{k}$ o sítio imediatamente a sua esquerda. Para $k$ fora do contorno, $\sigma_{k}^{*} \equiv \sigma_{k}$, enquanto que se $k$ e $\hat{k}$ estão no interior do contorno, $\sigma_{k}^{*} \equiv-\sigma_{\hat{k}}$. No entanto, para $k$ dentro do contorno e $\hat{k}$ fora, $\sigma_{k}^{*} \equiv \sigma_{\hat{k}}$. O efeito dessa transformação é o de eliminar o contorno inteiramente, deslocando quaisquer contornos internos de um passo à direita.

Dessa forma, obtemos como limitante superior o peso energético

$$
\left\langle X_{j}^{b}\right\rangle \leq e^{-2 \beta|\gamma|} \frac{Z_{V(\gamma)}^{-}(h)}{Z_{V^{*}(\gamma)}^{+}(h)} .
$$

em que $V^{*}(\gamma)$ representa o volume $V(\gamma)$ transladado de um passo à direita na rede.

É simples verificarmos que

$$
Z_{V(\gamma)}^{-}(h) / Z_{V^{*}(\gamma)}^{+}(h) \leq e^{2 \beta \epsilon|\gamma|}
$$

e assim demonstrarmos existência de transição de fase para $\epsilon<1$ e para $\beta$ suficientemente grande.

Como vimos, a cada configuração $\sigma \in \Gamma^{-}(V(\gamma))(\Gamma(V(\gamma))$ o conjunto de todas as possíveis configurações) corresponde uma configuração $\sigma^{*} \in \Gamma^{+}\left(V^{*}(\gamma)\right)$ de tal modo que a contribuição para a razão $Z_{V(\gamma)}^{-}(h) / Z_{V^{\star}(\gamma)}^{+}(h)$ é dada por (na realidade, mapeamos $Z_{V(\gamma)}^{-}(h)$ em $Z_{V^{*}(\gamma)}^{+}(h)$ a menos de um termo de interação do campo com a fronteira)

$$
\exp \left\{-\beta\left[H_{V(\gamma)}^{-}(\sigma, h)-H_{V^{*}(\gamma)}^{+}\left(\sigma^{*}, h\right)\right]\right\}
$$


com $H(\sigma, h)$ como em (2.1.1) (as condições de fronteira que tratamos são internas a $\gamma)$.

Dado que $\sigma_{k}^{*}=-\sigma_{\hat{k}}$ para $k, \hat{k} \in V(\gamma)$, podemos reescrever $H_{V^{*}(\gamma)}^{+}\left(\sigma^{*}, h\right)$ como

$$
\begin{aligned}
H_{V^{*}(\gamma)}^{+}\left(\sigma^{*}, h\right) & =-\frac{1}{2} \sum_{|i-j|=1} \sigma_{i-1} \sigma_{j-1}-\epsilon \sum_{i \in V^{*}(\gamma)} h_{i}\left(-\sigma_{i-1}\right) \\
& + \text { interação com a fronteira } \gamma
\end{aligned}
$$

Pelo fato do campo escolhido ser alternado, temos que $h_{i}=-h_{i-1}$ para todo $i \in V^{*}(\gamma)$ e assim

$$
H_{V^{*}(\gamma)}^{+}\left(\sigma^{*}, h\right)=-\frac{1}{2} \sum_{|i-j|=1} \sigma_{i-1} \sigma_{j-1}-\epsilon \sum_{i \in V^{*}(\gamma)} h_{i-1}\left(\sigma_{i-1}\right)
$$

Podemos redefinir as somas em (2.4.20) de modo a obter $H_{V^{*}(\gamma)}^{+}\left(\sigma^{*}, h\right)=$ $H_{V(\gamma)}^{-}(\sigma, h)+$ interação com fronteira. Chegamos, portanto, ao resultado desejado. Compreendemos desse modo a escolha feita para a construção da configuração $C^{*}$ : ela é construída com o intuito de anular o efeito do campo no interior do contorno. Repare que se decidíssemos eliminar o contorno $\gamma$ transladando seus contornos internos em outra direção o efeito seria o mesmo. Note ainda que, devido à remoção do contorno $\gamma$ em $C$, os contornos internos possuem espaço para serem deslocados por uma unidade mesmo quando se encontram próximos a $\gamma$.

Nosso próximo passo seria estudar o sistema com o campo quase aleatório. Caso obtivéssemos uma estimativa análoga a (2.4.19), certamente asseguraríamos a persistência do argumento de Peierls. Infelizmente, a presença de blocos irregulares do campo em todas as escalas não nos permite um mapeamento direto entre as funções de partição como na situação com campo alternado. Desse modo, não conseguimos afirmar por este método se o modelo apresenta transição de fase.

Dadas a dificuldades apresentadas até então buscamos estudar o problema de transição de fase através do MHD, que definimos e discutimos amplamente no próximo capítulo. 


\section{Capítulo 3}

\section{Modelo hierárquico de Dyson}

Apresentamos neste capítulo uma generalização para duas dimensões do modelo hierárquico proposto por Dyson em [4] e em [5], conveniente ao nosso estudo de existência de uma fase ordenada no modelo de Ising ferromagnético com campo. O material desse capítulo se inspira em [17].

\subsection{Modelo sem campo}

O MHD é um modelo ferromagnético em $\mathbb{Z}$ sem invariância translacional, introduzido por Dyson a fim de exibir uma transição de fase em modelos ferromagnéticos unidimensionais com função de interação de decaimento lento, dada assintoticamente por

$$
J^{(d=1)}(k) \sim \frac{1}{|k|^{\gamma}}, \quad 1<\gamma \leq 2
$$

Consideramos aqui a versão bidimensional deste modelo. As configurações de variáveis de spin $\sigma=\left\{\sigma_{i}= \pm 1, i \in \Lambda\right\}$ são elementos de $\Omega_{\Lambda}=\{-1,1\}^{|\Lambda|}$, com $\Lambda$ um subconjunto de $\mathbb{Z}^{2}$. Introduzimos a energia de interação não estacionária $H_{\Lambda}$ como segue:

$$
H_{\Lambda}(\sigma):=-\frac{1}{2} \sum_{\substack{i, j \in \Lambda: \\ i<j}} J_{i j} \sigma_{i} \sigma_{j}
$$


definida pela matriz de interação $J=\left[J_{i j}\right]$, cujos elementos imitam a interação ferromagnética bidimensional

$$
J^{(d=2)}(|i-j|) \sim \frac{1}{|i-j|^{2 \gamma}}
$$

de dois corpos, substituíndo a distância euclideana $|i-j|$ pela distância hierárquica

$$
d_{L}(i, j):=\sqrt{2}\left(L^{n_{L}(i, j)-1}-1\right)
$$

com $L>1$ um número inteiro (escolhamo-lo, por simplicidade, igual a dois);

$$
n_{L}(i, j):=\inf \left\{n \in \mathbb{N}:\left[\frac{i}{L_{n}}\right]=\left[\frac{j}{L_{n}}\right]\right\}
$$

com $L_{n}=L^{n}$, denota o menor nível hierárquico que inclui ambos os sítios $i, j \in \Lambda$ e, para toda dupla real $x=\left(x_{1}, x_{2}\right), x_{i} \in \mathbb{R},[x]$ denota a dupla de inteiros $k=\left(k_{1}, k_{2}\right)$ tal que $k_{i} \leq x_{i}<k_{i}+1, i=1,2$.

A distância hierárquica (3.1.2) não é estacionária com respeito a um deslocamento arbitrário $a \in \mathbb{Z}^{2}$, mas sim com respeito a deslocamentos compatíveis com a hierarquia. Mais ainda, ela satisfaz a desigualdade

$$
|i-j|=\left(\sum_{l=1}^{2}\left(i_{l}-j_{l}\right)^{2}\right)^{\frac{1}{2}} \leq d_{L}(i, j)
$$

A medida de equilíbrio $\mu_{\Lambda}$ é a distribuição de probabilidade em $\Omega_{\Lambda}$ dada por

$$
\mu_{\Lambda}(\sigma):=\frac{1}{Z_{\Lambda}} e^{-\beta H_{\Lambda}(\sigma)}
$$

com o fator de normalização (função de partição)

$$
Z_{\Lambda}=\sum_{\sigma \in \Omega_{\Lambda}} e^{-\beta H_{\Lambda}(\sigma)}
$$


A esperança da função $f: \Omega_{\Lambda} \rightarrow \mathbb{R}$ com respeito a esta medida se escreve como

$$
\mu_{\Lambda}[f]:=\frac{1}{Z_{\Lambda}} \sum_{\sigma \in \Omega_{\Lambda}} f(\sigma) e^{-\beta H_{\Lambda}(\sigma)}
$$

A medida limite $\mu=\lim _{\Lambda \uparrow \mathbb{Z}^{2}} \mu_{\Lambda}$ existe se a grandeza

$$
\mathcal{J}_{0}:=\lim _{\Lambda \uparrow \mathbb{Z}^{2}} \sum_{j \in \Lambda} J_{0 j}
$$

for finita; isso implica $\gamma>1$ (o que representa uma interação de curto alcance; para $\gamma \leq 1$, a interação hierárquica não é convergente). Note que $\mathcal{J}_{i}=\mathcal{J}_{0}$ para todo $i \in \mathbb{Z}^{2}$ e $\int_{\mathbb{R}^{2}} \frac{1}{1+|x|^{2 \gamma}} d^{2} x<\infty$ se $\gamma>1$

\subsubsection{Critério para a existência de ordem de longo alcance}

Veremos agora que MHD exibe ordem de longo alcance caso $1<\gamma \leq 2$. Heuristicamente, para um sistema com condições de contorno livres e interação invariante por translação (e por hora sem campo magnético), a medida limite $\mu_{\Lambda}$ converge presumivelmente, no limite termodinâmico $\Lambda \uparrow \mathbb{Z}^{2}$, à fase de mistura $\mu=\frac{1}{2} \mu^{+}+\frac{1}{2} \mu^{-}$ $\left(\mu^{+}\right.$e $\mu^{-}$representam, respectivamente, as medidas estacionárias dos sistemas com condições de fronteira positiva e negativa). Isto significa que a densidade de magnetização

$$
m_{\Lambda}^{\text {livre }}=\frac{1}{|\Lambda|} \sum_{i \in \Lambda} \mu\left[\sigma_{i}\right]
$$

é identicamente nula (de modo uniforme; existe a simetria $\mu_{\Lambda}(\sigma)=\mu_{\Lambda}(-\sigma)$ ) em $\Lambda$, e não poderá ser empregada como parâmetro de ordem. Devemos assim buscar o parâmetro de ordem em outra grandeza, no caso, o quadrado da magnetização espontânea: Uma boa candidata é a covariância da "fase de mistura" (esta é uma função de spin não local, diferentemente da magnetização), que escrevemos como 


$$
\begin{aligned}
\mu\left[\sigma_{i} ; \sigma_{j}\right]:= & \mu\left[\sigma_{i} \sigma_{j}\right]-\mu\left[\sigma_{i}\right] \mu\left[\sigma_{j}\right] \\
= & \frac{1}{2}\left(\mu^{+}\left[\sigma_{i} ; \sigma_{j}\right]+\mu^{-}\left[\sigma_{i} ; \sigma_{j}\right]\right) \\
& +\frac{1}{4}\left(\mu^{+}\left[\sigma_{i}\right]-\mu^{-}\left[\sigma_{i}\right]\right)\left(\mu^{+}\left[\sigma_{j}\right]-\mu^{-}\left[\sigma_{j}\right]\right) .
\end{aligned}
$$

Tendo em vista o decaimento da função de correlação para uma fase pura $\left(\mu^{ \pm}\left[\sigma_{i} ; \sigma_{j}\right] \rightarrow 0\right),(3.1 .7)$ se comporta como

$$
\mu\left[\sigma_{i} ; \sigma_{j}\right] \rightarrow \frac{1}{4}\left(\mu^{+}\left[\sigma_{i}\right]-\mu^{-}\left[\sigma_{i}\right]\right)\left(\mu^{+}\left[\sigma_{j}\right]-\mu^{-}\left[\sigma_{j}\right]\right)
$$

à medida que $d_{L}(i, j) \rightarrow \infty$.

Como conseqüência, (3.1.8) vai a zero $\operatorname{com} d_{L}(i, j) \rightarrow \infty$ se e somente se não ocorre uma quebra espontânea de simetria (ou seja, $\mu^{ \pm}\left[\sigma_{i}\right]=0$ ). Este argumento heurístico nos conduz ao seguinte critério para determinação de ordem de longo alcance:

Definição 3.1.1 Diz-se que um sistema de spins exibe ordem de longo alcance (OLA) se o limite

$$
R_{\mu}:=\lim _{\Lambda \uparrow \mathbb{Z}^{2}} \frac{1}{|\Lambda|^{2}} \sum_{i, j \in \Lambda} \mu\left[\sigma_{i} ; \sigma_{j}\right]
$$

existe e for positivo.

Quando existe a simetria $\mu_{\Lambda}(\sigma)=\mu_{\Lambda}(-\sigma)$ (como no MHD com condição de fronteira livre), $\mu\left[\sigma_{i}\right]=0$ e existe ordem de longo alcance se

$$
m^{2}:=\lim _{\Lambda \uparrow Z^{2}} \mu\left[\left(\frac{1}{|\Lambda|} \sum_{i \in \Lambda} \sigma_{i}\right)^{2}\right]>0
$$

justamente a variância da média dos spins em $\Lambda$.

Vale destacar que nesta situação o parâmetro de ordem de longo alcance é justamente a variância da média dos spins em $\Lambda$ (uma vez que (3.1.6) se anula). 


\subsubsection{Relação de recorrência para o parâmetro de ordem $m^{2}$}

A fim de verificarmos se o MHD sem campo apresenta ordem de longo alcance, é conveniente definirmos as chamadas variáveis de bloco. Fixemos a escala obedecendo a lei $L_{n}=L^{n}$, com $L=2$; façamos $N \in \mathbb{N}$ grande e definamos uma seqüência de subconjuntos de $(\mathbb{Z} \cup\{-1 / 2\}) \times(\mathbb{Z} \cup\{-1 / 2\})$ :

$$
\Lambda_{n}:=\left(\left[-2^{n-1}, 2^{n-1}-1\right] \cap \mathbb{Z}\right)^{2}
$$

para $n=1,2, \ldots, N$ e $\Lambda_{0}:=\{(-1 / 2,-1 / 2)\}$ para $n=0$. Denotamos $\Lambda=\Lambda_{N}$.

Dados $n=1,2, \ldots, N$ e $j \in \Lambda_{N-n}$, seja $\sigma_{n, j}$ a $(n, j)$-variável de bloco, definida indutivamente por

$$
\begin{aligned}
\sigma_{n, j}:= & \sigma_{n-1,\left(2 j_{1}, 2 j_{2}\right)}+\sigma_{n-1,\left(2 j_{1}, 2 j_{2}+1\right)}+\sigma_{n-1,\left(2 j_{1}+1,2 j_{2}\right)} \\
& +\sigma_{n-1,\left(2 j_{1}+1,2 j_{2}+1\right)}
\end{aligned}
$$

com $\sigma_{0, j} \equiv \sigma_{j}$. A variável de bloco $\sigma_{n, j}$ é assim a soma de $2^{2 n}$ variáveis aleatórias consecutivas:

$$
\sigma_{n, j}:=\sum_{l, m=0}^{2^{n}-1} \sigma_{2^{n}\left(j_{1}, j_{2}\right)+(l, m)}
$$

Com estas notações, a energia total de interação (3.1.1) pode ser reescrita como

$$
H_{\Lambda_{N}}(\sigma)=-\frac{1}{2} \sum_{n=0}^{N-1} \xi_{n} \sum_{j \in \Lambda_{N-n-1}} I_{n, j}(\sigma)
$$

$\operatorname{com} \xi_{n}=c_{n} 2^{-2 \gamma n}$. O parâmetro $c_{n}=c_{n}(\gamma)$ é tal que $c_{n}=1$ se $1<\gamma<2$ e $c_{n}=\log n, n \geq 1$, com $c_{0}=1$, se $\gamma=2$ (a correção logarítmica no caso $\gamma=2$ (Modelo de Anderson) visa assegurar a existência de ordem de longo alcance; vide [5] para os detalhes e [10] para uma prova da existência de ordem). Note que 
$\mathcal{J}_{0}<\infty$ para $1<\gamma \leq 2$, i.e., a interação é convergente. Fizemos em (3.1.12) uso da notação

$$
\begin{aligned}
I_{n, j}(\sigma) \equiv & \sigma_{n,\left(2 j_{1}, 2 j_{2}\right)}\left(\sigma_{n,\left(2 j_{1}+1,2 j_{2}\right)}+\sigma_{n,\left(2 j_{1}, 2 j_{2}+1\right)}+\sigma_{n,\left(2 j_{1}+1,2 j_{2}+1\right)}\right) \\
& +\sigma_{n,\left(2 j_{1}+1,2 j_{2}\right)}\left(\sigma_{n,\left(2 j_{1}, 2 j_{2}+1\right)}+\sigma_{n,\left(2 j_{1}+1,2 j_{2}+1\right)}\right) \\
& +\sigma_{n,\left(2 j_{1}, 2 j_{2}+1\right)} \sigma_{n,\left(2 j_{1}+1,2 j_{2}+1\right)}
\end{aligned}
$$

que representa a interação aos pares entre quatro sítios vizinhos da (n-1)-ésima hierarquia, sem repetições. Podemos reescrever (3.1.12) como

$$
\begin{aligned}
H_{\Lambda_{N}}(\sigma)= & H_{\Lambda_{N-1}^{++}}(\sigma)+H_{\Lambda_{N-1}^{+-}}(\sigma)+H_{\Lambda_{N-1}^{-+}}(\sigma) \\
& +H_{\Lambda_{N-1}^{--}}(\sigma)-\frac{1}{2} \xi_{N-1} I_{N-1,-1 / 2}(\sigma),
\end{aligned}
$$

dadas as definições

$$
\begin{aligned}
\Lambda_{k}^{++} & :=\left(\left[0,2^{k-1}-1\right] \cap \mathbb{Z}\right)^{2}, \\
\Lambda_{k}^{+-} & :=\left(\left[0,2^{k-1}-1\right] \cap \mathbb{Z}\right) \times\left(\left[-2^{k-1},-1\right] \cap \mathbb{Z}\right), \\
\Lambda_{k}^{-+} & :=\left(\left[-2^{k-1},-1\right] \cap \mathbb{Z}\right) \times\left(\left[0,2^{k-1}-1\right] \cap \mathbb{Z}\right), \\
\Lambda_{k}^{--} & :=\left(\left[-2^{k-1},-1\right] \cap \mathbb{Z}\right)^{2}
\end{aligned}
$$

para todo $k \in \mathbb{N}$.

É útil enfatizarmos que a diferença entre energias em diferentes escalas produz apenas fatores locais. Localidade é a característica principal dos modelos hierárquicos.

A equação (3.1.14) nos permite escrever o valor esperado de $g: \Omega_{\Lambda_{N}} \rightarrow \mathbb{R}$ como

$$
\mu_{\Lambda_{N}}[g]=\frac{\mu_{\Lambda_{N-1,0}}\left[g \exp \left\{(\beta / 2) \xi_{N-1} I_{N-1,-1 / 2}(\sigma)\right\}\right]}{\mu_{\Lambda_{N-1,0}}\left[\exp \left\{(\beta / 2) \xi_{N-1} I_{N-1,-1 / 2}(\sigma)\right\}\right]}
$$

$\operatorname{com} \mu_{\Lambda_{k, 0}} \equiv \mu_{\Lambda_{k}^{++}, \Lambda_{k}^{+-}, \Lambda_{k}^{-+}, \Lambda_{k}^{--}}=\mu_{\Lambda_{k}^{++}} \cdot \mu_{\Lambda_{k}^{+-}} \cdot \mu_{\Lambda_{k}^{-+}} \cdot \mu_{\Lambda_{k}^{--}}$

Definamos 


$$
f_{N}(n):=\frac{1}{2^{4 n}} \mu_{\Lambda_{N}}\left[\sigma_{n, j}^{2}\right]
$$

e $f(n):=\lim _{N \rightarrow \infty} f_{N}(n)$ para todo $n \in\{0,1, \ldots, N\}$ (note que (3.1.16) independe de $\left.j \in \Lambda_{N-n}\right)$.

Portanto, o parâmetro $m^{2}$ definido pelo limite (3.1.9), aproximando-se de $\mathbb{Z}^{2}$ pela subseqüência (3.1.10), pode ser escrito como

$$
m^{2}=\lim _{n \rightarrow \infty} f(n)
$$

De (3.1.11) e da definição (3.1.16) temos

$$
\begin{aligned}
f_{N}(N)= & \frac{1}{2^{4 N}} \mu_{\Lambda_{N}}\left[\sigma_{N,(-1 / 2,-1 / 2)}^{2}\right] \\
= & \frac{1}{2^{4 N}} \mu_{\Lambda_{N}}\left[\sigma_{N-1,(-1,-1)}^{2}+\sigma_{N-1,(-1,0)}^{2}+\sigma_{N-1,(0,-1)}^{2}+\sigma_{N-1,(0,0)}^{2}\right] \\
& +\frac{1}{2^{4 N-1}} \mu_{\Lambda_{N}}\left[I_{N-1,-1 / 2}(\sigma)\right] \\
= & \frac{1}{4} f_{N}(N-1)+\frac{1}{2^{4 N-1}} \mu_{\Lambda_{N}}\left[I_{N-1,-1 / 2}(\sigma)\right]
\end{aligned}
$$

uma vez que, por simetria da medida, $\mu_{\Lambda_{N}}\left[\sigma_{N-1,(i, j)}^{2}\right]=2^{4(N-1)} f_{N}(N-1)$ para todo $i, j \in\{0,1\}$.

Tudo isto nos deixa em posição de estabeler o resultado de Dyson para a existência de ordem de longo alcance (trata-se, na realidade, da nossa generalização deste resultado).

\subsubsection{Teorema principal e desigualdades de Griffiths}

Teorema 3.1.2 (Dyson(71)[5]) Seja $\mu=\lim _{N \rightarrow \infty} \mu_{\Lambda_{N}}$ a medida limite do MHD com $1<\gamma<2$, e seja $m^{2}$ dado por (3.1.9). Então,

$$
m^{2}>\frac{x_{1}\left(\beta^{\star}\right)}{a^{\star}}
$$


com $x_{1}(\beta)$ definido por $(3.1 .34)$, segue se $\beta>\beta^{\star} \approx 37,4$. Existe portanto, pelo critério 3.1.1, ordem de longo alcance.

A prova do Teorema 3.1.2 é uma conseqüência imediata das desigualdades de Griffiths e de um lema probabilístico que está por vir. Discutamos em primeiro lugar as desigualdades.

Definição 3.1.3 Um conjunto de variáveis aleatórias $\left\{\omega_{j}\right\}_{j \in \mathbb{Z}^{2}}$ é dito satisfazer as desigualdades FKG se

$$
\mu[f ; g]:=\mu[f g]-\mu[f] \mu[g] \geq 0
$$

segue para quaisquer funções crescentes $f, g$.

Estas desigualdades, satisfeitas pelos modelos ferromagnéticos (vide [7]), implicam que todas as correlações são não-negativas.

Sejam $\mu_{1}$ e $\mu_{2}$ duas medidas de Gibbs finitas com condições de fronteira livres em $\Lambda$ e com matrizes de interação $J_{\Lambda}^{1}$ e $J_{\Lambda}^{2}$ respectivamente. Suponhamos que $J_{\Lambda}^{1} \leq J_{\Lambda}^{2}$ (isto é, $\left(J_{\Lambda}^{1}\right)_{i j} \leq\left(J_{\Lambda}^{2}\right)_{i j}$ para todos $i, j \in \Lambda$ ). Então, pelas desigualdades de Griffiths,

$$
\mu_{1}\left[\sigma_{A}\right] \leq \mu_{2}\left[\sigma_{A}\right]
$$

segue com $\sigma_{A}=\prod_{i \in A} \sigma_{i}$ para todo $A \subset \Lambda$. Elas essencialmente dizem que o fortalecimento das ligações ferromagnéticas de um modelo leva a um aumento dessas correlações.

Usamos as desigualdades de Griffiths para dois propósitos distintos. Em primeiro lugar, (3.1.18) é usado para comparar o MHD com um modelo ferromagnético geral (fg) em $\mathbb{Z}^{2}$ com interações $J_{i j}^{f g}=1 /|i-j|^{2 \gamma}$, com $1<\gamma \leq 2$. De (3.1.3) obtemos

$$
J_{i j}^{D h} \leq J_{i j}^{f g}
$$

Em vista da definição (3.1.9), de (3.1.18) e de (3.1.19) temos 


$$
m_{D h}^{2} \leq m_{f g}^{2}
$$

Extraímos de (3.1.20) as seguinte conclusão: OLA para o MHD implica OLA para um modelo ferromagnético geral.

A segunda aplicação da desigualdade de Griffiths compara dois MHDs definidos em um volume $\Lambda$ e $\Lambda^{\prime} \supseteq \Lambda$, respectivamente. Escrevemos a interação $J_{\Lambda}$ como uma matriz $\left|\Lambda^{\prime}\right| \times\left|\Lambda^{\prime}\right|$ cujas entradas $\left\{i, j \in \Lambda^{\prime}: i \in \Lambda^{\prime} \backslash \Lambda\right.$ e/ou $\left.j \in \Lambda^{\prime} \backslash \Lambda\right\}$ são iguais a zero. Claramente

$$
J_{\Lambda} \leq J_{\Lambda^{\prime}}
$$

Da definição (3.1.16) e de (3.1.21) concluímos

$$
f_{N}(n)=\frac{1}{2^{4 n}} \mu_{\Lambda_{N}}\left[\sigma_{n, j}^{2}\right] \geq \frac{1}{2^{4 n}} \mu_{\Lambda_{N-1}}\left[\sigma_{n, j}^{2}\right]=f_{N-1}(n)
$$

Por outro lado, usamos a monotonicidade de $f_{N}(n)$ para obter

$$
f_{N}(n) \leq f_{N}(n-1)
$$

já que

$$
\begin{aligned}
\left(\frac{\sigma_{n, j}}{2^{2 n}}\right)^{2} & =\frac{1}{64}\left(\frac{1}{2^{2(n-1)}} \sum_{0 \leq i_{1}, i_{2} \leq 1} \sigma_{n-1,\left(2 j_{1}+i_{1}, 2 j_{2}+i_{2}\right)}\right)^{2} \\
& \leq \frac{1}{4} \sum_{0 \leq i_{1}, i_{2} \leq 1}\left(\frac{\sigma_{n-1,\left(2 j_{1}+i_{1}, 2 j_{2}+i_{2}\right)}}{2^{2(n-1)}}\right)^{2}
\end{aligned}
$$

segue para todo $\sigma \in\{-1,1\}^{|\Lambda|}$ (a função $f_{N}$ é independente do sítio que define o bloco de ordem $N$ ). Fizemos na expressão acima uso da desigualdade $2 \sigma_{n-1, i} \sigma_{n-1, j} \leq\left(\sigma_{n-1, i}\right)^{2}+\left(\sigma_{n-1, j}\right)^{2}$.

Como $0 \leq f_{N}(n) \leq 1$ (conseqüência direta da definição), $f_{N}(n)$ tende a um limite $f(n)$ quando $N \rightarrow \infty$, o que por sua vez, de (3.1.23), tende ao limite $m^{2}$ quando $n \rightarrow \infty$. 


\subsubsection{Prova do Teorema 3.1.2}

Necessitamos de uma desigualdade para o termo $\mu_{\Lambda_{N}}\left[I_{N-1,-1 / 2}(\sigma)\right]$ presente em (3.1.17), a relação de recorrência para o parâmetro de ordem $m^{2}$. Para tanto, façamos uso do seguinte lema probabilístico (generalização de nossa autoria do lema desenvolvido em [4] para $d \geq 2$ e tamanho do bloco $L \geq 2$ ).

Lema 3.1.4 Seja $X=\left(X_{\alpha}\right)_{\alpha=1}^{d}$ um vetor de variáveis aleatórias com distribuições de probabilidades simétricas em relação à origem $\left(\mathbb{E} X_{\alpha}^{2 l-1}=0, l \in \mathbb{N}\right)$, tal que $\mathbb{E}\left[X_{\alpha} X_{\beta}\right]=0$ para $\alpha \neq \beta$ e $\mathbb{E}\left[(1 \cdot X) e^{a \cdot X}\right]<\infty$. Então, para cada $a_{\alpha}>0$, $\alpha \in\{1, \ldots, \delta\}$,

$$
\frac{\mathbb{E}\left[(1 \cdot X) e^{a \cdot X}\right]}{\mathbb{E}\left[e^{a \cdot X}\right]} \geq(1 \cdot \bar{R}) \tanh [\bar{R} a]
$$

segue com $\bar{R}:=\operatorname{diag}\left[\bar{\lambda}_{\alpha}\right]$, onde $\bar{\lambda}_{\alpha}^{2}:=\mathbb{E} X_{\alpha}^{2}, 1:=(1, \ldots, 1)$ e $\delta$ o número de pares presentes em um bloco de comprimento L. Em (3.1.24), uma função de vetores $f(v)=\left(f\left(v_{1}\right), \ldots, f\left(v_{d}\right)\right)$ é interpretada como o vetor de componentes dados pela aplicação da função sobre cada componente do vetor $v$; o produto escalar é definido por $f \cdot g=\sum_{\alpha} f_{\alpha} g_{\alpha}$.

Observação 3.1.5 Vale notar que não é possivel aplicar o lema original de Dyson nesta situação geral, uma vez que este subestima o membro esquerdo de (3.1.24), o que não nos permite concluir a existência de ordem de longo alcance através desse método.

Prova. A prova do Lema 3.1.4 se encontra desenvolvida em detalhe no apêndice A.

Definindo $X$ como o vetor em $\mathbb{R}^{6}$ tal que $1 \cdot X:=I_{N-1,-1 / 2}(\sigma)$, o vetor $a \in \mathbb{R}^{6}$ de componentes $a_{\alpha}=\beta \xi_{N-1} / 2$, e a distribuição de probabilidade

$$
P_{X}(x)=\sum_{\sigma} \prod_{\alpha=1}^{6} \mu_{\Lambda_{N-1}^{++}}(\sigma) \mu_{\Lambda_{N-1}^{+-}}(\sigma) \mu_{\Lambda_{N-1}^{-+}}(\sigma) \mu_{\Lambda_{N-1}^{--}}(\sigma) \delta_{x_{\alpha}, X_{\alpha}}
$$


podemos reescrever $\mu_{\Lambda_{N}}\left[I_{N-1,-1 / 2}(\sigma)\right]$, em vista de (3.1.15), como

$$
\begin{aligned}
\mu_{\Lambda_{N}}\left[I_{N-1,-1 / 2}(\sigma)\right] & =\frac{\sum_{\sigma} I_{N-1,-1 / 2}(\sigma) \exp \left\{\frac{\beta \xi_{N-1}}{2} I_{N-1,-1 / 2}(\sigma)\right\} \mu_{\Lambda_{N}}(\sigma)}{\sum_{\sigma} \exp \left\{\frac{\beta \xi_{N-1}}{2} I_{N-1,-1 / 2}(\sigma)\right\} \mu_{\Lambda_{N}}(\sigma)} \\
& =\frac{\mathbb{E}\left[(1 \cdot X) e^{a \cdot X}\right]}{\mathbb{E}\left[e^{a \cdot X}\right]}
\end{aligned}
$$

Note que $\mathbb{E}\left[X_{\alpha} X_{\beta}\right]=0$ segue para $\alpha \neq \beta$, com $P_{X}$ dado por (3.1.25). De (3.1.24) e (3.1.26) chegamos a

$$
\mu_{\Lambda_{N}}\left[I_{N-1,-1 / 2}(\sigma)\right] \geq(1 \cdot \bar{R}) \tanh (\bar{R} a)
$$

$\operatorname{com} \bar{R}:=\operatorname{diag}\left[\bar{\lambda}_{\alpha}\right]$ e $\bar{\lambda}_{\alpha}^{2}:=\mathbb{E} X_{\alpha}^{2}$ (esta esperança se dá em termos da medida de probabilidade produto dos blocos das respectivas variáveis de spin que constituem $\left.X_{\alpha}^{2}\right)$.

Definamos

$$
\begin{aligned}
\bar{\lambda}^{2} & =\sum_{\sigma, \sigma^{\prime}}\left(\sigma^{\prime} \sigma\right)^{2} \mu_{\Lambda_{N-1}}\left(\sigma^{\prime}\right) \mu_{\Lambda_{N-1}}(\sigma) \\
& =\sum_{\sigma} \sigma^{2} \mu_{\Lambda_{N-1}}(\sigma) \sum_{\sigma^{\prime}}\left(\sigma^{\prime}\right)^{2} \mu_{\Lambda_{N-1}}\left(\sigma^{\prime}\right)=\bar{\lambda}_{\alpha}^{2}
\end{aligned}
$$

para todo $\alpha \in\{1, \ldots, 6\}$. Temos, por simetria, a identidade $\bar{\lambda}_{\alpha}^{2}=\bar{\lambda}_{\beta}^{2}$ para todo $\alpha, \beta \in\{1, \ldots, 6\}$, uma vez que as medidas de probabilidade de cada um dos blocos $\Lambda_{N-1}$ são idênticas.

Da definição (3.1.28) e da relação (3.1.27) concluímos

$$
\mu_{\Lambda_{N}}\left[I_{N-1,-1 / 2}(\sigma)\right] \geq 6 \bar{\lambda} \tanh \left(\frac{\beta \xi_{N-1}}{2} \bar{\lambda}\right)
$$

$\operatorname{com} \bar{\lambda}=2^{4(N-1)} f_{N-1}(N-1)$, de acordo com a definição (3.1.16). Note que o fator 6 , igual ao número de pares presentes em um bloco de comprimento $L=2$, é fundamental para a obtenção da relação de recorrência para $f_{N}(N)$ (caso utilizássemos 
o Lema original de Dyson obteríamos um fator $\sqrt{6}$, insuficiente para concluir o argumento).

Finalmente, extraímos de (3.1.17), (3.1.22) e (3.1.29) a desigualdade

$$
f_{N}(N) \geq \frac{f_{N-1}(N-1)\left(1-\frac{1}{2} \exp \left\{-\beta c_{N-1}(\gamma) 2^{(4-2 \gamma)(N-1)} f_{N-1}(N-1)\right\}\right)}{1+\exp \left\{-\beta c_{N-1}(\gamma) 2^{(4-2 \gamma)(N-1)} f_{N-1}(N-1)\right\}}
$$

onde usamos a definição $\xi_{N-1}(\gamma):=\frac{c_{N-1}(\gamma)}{2^{2 \gamma(N-1)}}$ e a identidade

$$
1+3 \tanh x=4 \frac{1-\frac{1}{2} e^{-2 x}}{1+e^{-2 x}}
$$

Seja $x_{N}=x_{N}(\gamma), N \in \mathbb{N}$, uma seqüência de números reais satisfazendo a desigualdade (3.1.30), sujeita à condição inicial $x_{1}=f_{1}(1)$. Desejamos obter um limitante inferior de $x_{N}$ uniforme em $N$ e $\gamma$. Sendo a função

$$
g_{N}(\gamma)=\frac{1-\frac{1}{2} \exp \left\{-\beta c_{N-1}(\gamma) 2^{(4-2 \gamma)(N-1)} x_{N-1}\right\}}{1+\exp \left\{-\beta c_{N-1}(\gamma) 2^{(4-2 \gamma)(N-1)} x_{N-1}\right\}}
$$

monótona decrescente em $\gamma, 1 \leq \gamma \leq 2$, é suficiente obter um limitante inferior para o caso $\gamma=2$. Provemos nossa afirmação. Como

$$
c_{N-1}(\gamma)= \begin{cases}1 & \text { se } 1 \leq \gamma<2 \\ \log (N-1) & \text { se } \gamma=2\end{cases}
$$

temos que $c_{N-1}^{\prime}(\gamma)=0$ para $\gamma \in[1,2)$, e portanto

$$
g_{N}^{\prime}(\gamma)=\frac{-3 \beta x_{N-1}(N-1)(\log 2) 2^{(4-2 \gamma)(N-1)} \exp \left\{-2^{(4-2 \gamma)(N-1)} \beta x_{N-1}\right\}}{\left(1+\exp \left\{-2^{(4-2 \gamma)(N-1)} \beta x_{N-1}\right\}\right)^{2}} \leq 0
$$

o que prova nossa afirmação. Dessa forma,

$$
\begin{aligned}
g_{N}(\gamma) & \geq g_{N}(2)=\frac{1-\frac{1}{2} \exp \left\{-\beta \log (N-1) x_{N-1}\right\}}{1+\exp \left\{-\beta \log (N-1) x_{N-1}\right\}} \\
& =\frac{1-\frac{1}{2}(N-1)^{-\beta x_{N-1}}}{1+(N-1)^{-\beta x_{N-1}}} .
\end{aligned}
$$


Das relações (3.1.30) e (3.1.32) obtemos

$$
x_{N} \geq x_{N-1}\left(\frac{1-\frac{1}{2}(N-1)^{-\beta x_{N-1}}}{1+(N-1)^{-\beta x_{N-1}}}\right) \text {, }
$$

com

$$
\begin{aligned}
x_{1} & =\frac{\sum_{\sigma_{1}, \sigma_{2}, \sigma_{3}, \sigma_{4} \in\{-1,1\}}\left(\frac{\sigma_{1}+\sigma_{2}+\sigma_{3}+\sigma_{4}}{4}\right)^{2} e^{\frac{\beta}{4}\left[\sigma_{1}+\sigma_{2}+\sigma_{3}+\sigma_{4}\right]^{2}}}{\sum_{\sigma_{1}, \sigma_{2}, \sigma_{3}, \sigma_{4} \in\{-1,1\}} e^{\frac{\beta}{4}\left[\sigma_{1}+\sigma_{2}+\sigma_{3}+\sigma_{4}\right]^{2}}} \\
& =\frac{e^{\beta}+e^{4 \beta}}{3+4 e^{\beta}+e^{4 \beta}} .
\end{aligned}
$$

Sendo $x_{1}(\beta)$ uma função monótona crescente (a prova é direta e será omitida), segue $x_{1}(\beta) \geq x_{1}(0)=1 / 4$. Como

$$
\lim _{\beta \rightarrow \infty} x_{1}(\beta)=\lim _{\beta \rightarrow \infty} \frac{e^{\beta}+e^{4 \beta}}{3+4 e^{\beta}+e^{4 \beta}}=1
$$

concluímos que $1 / 4 \leq x_{1}(\beta) \leq 1$.

Por fim, resta-nos mostrar que a função

$$
h_{K}(y):=\frac{1-\frac{1}{2} K^{-\beta y}}{1+K^{-\beta y}}
$$

$K \in \mathbb{N}$, é também monótona crescente.

Temos

$$
\frac{d}{d y} h_{K}=\frac{\frac{3}{2} \beta(\log K) K^{-\beta y}}{\left(1+K^{-\beta y}\right)^{2}}>0
$$

para todo $y \geq 0$ e todo $K \in \mathbb{N}$. Assim sendo, $h_{K}(y)$ é monótona e satisfaz a desigualdade $h_{K}(y) \geq h_{K}(0)=1 / 4$.

Usemos agora indução matemática a fim de obter um limitante inferior de $\beta$ $\left(\beta_{c}\right)$. Vamos supor que 


$$
x_{k} \geq \frac{x_{1}}{a} \geq \frac{1}{4 a}
$$

seja válida para $k=1,2, \ldots, N-1$ e para algum $a>0$ (determinado de modo a otimizar $\beta_{c}$ ). Aplicando (3.1.32) sucessivamente e substituíndo $x_{k}$ pelo seu limitante inferior $x_{1} / a$ obtemos, dada a monotonicidade de $h_{k-1}(y)$,

$$
\begin{aligned}
x_{N} & \geq x_{1} \prod_{l=1}^{N-1} \frac{1-\frac{1}{2} l^{-\beta x_{1} / a}}{1+l^{-\beta x_{1} / a}} \\
& =x_{1} \exp \left\{\sum_{l=1}^{N-1}\left[\log \left(1-\frac{l^{-\beta x_{1} / a}}{2}\right)\right]-\left[\log \left(1+l^{-\beta x_{1} / a}\right)\right]\right\} .
\end{aligned}
$$

Das desigualdades $\log (1+x) \leq x$ para $x \in \mathbb{R}_{+}$e $\log (1-x) \geq-2(\log 2) x$ para $0<x \leq 1 / 2$, obtemos de (3.1.37) (note que $\frac{1}{2} l^{-\beta x_{1} / a} \leq \frac{1}{2} l^{-\beta / 4 a} \leq \frac{1}{2}$ para todo $\beta$ e todo $a>0$ ) e da hipótese indutiva

$$
\begin{aligned}
x_{N} & \geq x_{1} \exp \left\{-(1+\log 2) \sum_{l=1}^{N-1} l^{-\beta x_{1} / a}\right\} \\
& \geq x_{1} \exp \left\{-(1+\log 2)\left(1+\int_{1}^{\infty} \frac{d y}{y^{\beta x_{1} / a}}\right)\right\} \\
& =x_{1} \exp \left\{-(1+\log 2) \frac{1}{1-a / \beta x_{1}}\right\} \geq \frac{x_{1}}{a} .
\end{aligned}
$$

Temos de (3.1.38) que

$$
\exp \left\{-(1+\log 2) \frac{1}{1-a / \beta x_{1}}\right\} \geq \frac{1}{a}=\exp \{-\log a\}
$$

o que implica que

$$
\beta \geq \frac{1}{x_{1}} \frac{a}{1-\frac{1+\log 2}{\log a}}=\frac{1}{x_{1}} u(a)
$$

Busquemos o ponto de mínimo da função $u(a)$ : 


$$
\frac{d u}{d a}\left(a^{\star}\right)=\frac{1}{\left(1-\frac{1+\log 2}{\log a^{\star}}\right)^{2}}\left\{1-\frac{1+\log 2}{\log a^{\star}}-\frac{1+\log 2}{\left(\log a^{\star}\right)^{2}}\right\}=0,
$$

de onde extraímos

$$
a^{\star}=\exp \left\{\frac{1+\log 2}{2}+\frac{1+\log 2}{2}\left(1+\frac{4}{1+\log 2}\right)^{1 / 2}\right\} \approx 11,01
$$

conduzindo-nos ao valor $u\left(a^{\star}\right) \approx 37,42$. A verificação de que $a^{\star}$ se trata de um mínimo de $u(a)$ é direta e será omitida.

Obtemos uma estimativa $\beta^{\star}$ para o inverso da temperatura crítica $\beta_{c}$ resolvendo (3.1.39) para $\beta$ como igualdade, com $a=a^{\star}$. Numericamente, $\beta^{\star} \approx u\left(a^{\star}\right) \approx 37,42$.

Todavia, de (3.1.23)

$$
f_{N}(p) \geq f_{N}(N) \equiv x_{N}>\frac{x_{1}\left(\beta^{\star}\right)}{a^{\star}}
$$

para todo $N \geq p \geq 1$ e todo $\beta>38$, o que, em vista de $m^{2}=\lim _{n \rightarrow \infty} \lim _{N \rightarrow \infty} f_{N}(n)$, implica em $m^{2}>x_{1}\left(\beta^{\star}\right) / a^{\star} \approx 1 / 12$. Concluímos, portanto, a prova do Teorema 3.1.2.

\subsection{Modelo com campo externo quase aleatório}

Na presente seção, apresentamos a generalização do modelo exibido na Seção 3.1 na presença do campo quase aleatório (com a estrutura apresentada na Seção 2.3). Não é possível demostrar a existência de ordem de longo alcance pelo método empregado na ausência de campo, a menos que sua intensidade nas regiões irregulares varie com a escala.

Propomos então uma configuração cujas regiões irregulares (no sentido apresentado na Seção 2.3) sejam esparsas (isto é, aceleramos o crescimento das escalas e mantemos o número de blocos irregulares em cada uma delas igual a dois). Se o 
crescimento das escalas for ao menos exponencial, então podemos, como na ausência de campo, estabelecer a existência da fase ferromagnética. Os detalhes seguem a seguir.

\subsubsection{Na ausência de esparsidade}

O modelo com campo externo sem esparsidade apresenta apenas algumas alterações com respeito ao modelo discutido na seção anterior. A principal delas é a inclusão, no hamiltoniano (3.1.1), do termo de interação entre o campo (considerado como um bloco configuracional $N$-admissível) e os spins da rede bidimensional $\Lambda_{N} \subset \mathbb{Z}^{2}$, descrito por uma interação pontual. Reescrevemos (3.1.12) como

$$
H_{\Lambda_{N}}(\sigma, h)=-\frac{1}{2} \sum_{n=0}^{N-1} \xi_{n} \sum_{j \in \Lambda_{N-n-1}} I_{n, j}(\sigma)-\epsilon \sum_{i \in \Lambda_{N}} h_{i} \sigma_{i}
$$

com $I_{n, j}(\sigma)$ definido por (3.1.13).

Dada a notável simetria apresentada pelo campo introduzimos novas variáveis de spin, de soma e de subtração dos blocos irregulares, definidas pelas expressões

$$
\begin{aligned}
\sigma_{N-1}^{+} & :=\sigma_{\Lambda_{N-1}^{++}}+\sigma_{\Lambda_{N-1}^{--}}, \\
\sigma_{N-1}^{-} & :=\sigma_{\Lambda_{N-1}^{++}}-\sigma_{\Lambda_{N-1}^{--}} .
\end{aligned}
$$

Com as definições (3.2.2) e (3.2.3) e a estrutura $N$-admissível do campo podemos reescrever o termo de interação com o campo em (3.2.1) como

$$
\sum_{i \in \Lambda_{N}} h_{i} \sigma_{i}=\sigma_{N-1}^{-}+\sum_{i \leq 2} \sigma_{N-2, i}^{-}+\cdots+\sum_{i \leq 2^{N-1}} \sigma_{1, i}^{-}+\sum_{i \leq 2^{N-1}}\left(\sigma_{0, i}^{-}+\widetilde{\sigma}_{0, i}^{-}\right)
$$

uma vez que os termos envolvendo as variáveis de soma se anulam (os blocos irregulares estão presentes sempre aos pares e com sinais opostos). O bloco $\Lambda_{N}$ é composto por dois blocos $(N-1)$-irregulares (com sinais opostos) e dois blocos 
$(N-1)$-admissíveis; há, portanto, uma única variável de subtração, $\sigma_{N-1}^{-}$. À medida que diminuímos o nível hierárquico, surgem mais blocos irregulares (sempre aos pares) em $\Lambda_{N}$, de modo que no nível $k=2$ há $2^{N-1}$ variáveis $\sigma_{1, j}^{-}, j=1, \ldots, 2^{N-1}$. O caso $k=1$ é particular, uma vez que nos blocos admissíveis do primeiro nível hierárquico o campo é alternado nos quatro sítios. Devemos então definir duas variáveis de subtração, $\sigma_{0, j}^{-}$e $\widetilde{\sigma}_{0, l}^{-}, j, l=1, \ldots, 2^{N-1}$.

Podemos, como em (3.1.14), decompor o hamiltoniano (3.2.1) em seus constituíntes da $(N-1)$-ésima escala:

$$
\begin{aligned}
H_{\Lambda_{N}}(\sigma, h)= & H_{\Lambda_{N-1}^{++}}(\sigma)+H_{\Lambda_{N-1}^{+-}}(\sigma)+H_{\Lambda_{N-1}^{-+}}(\sigma, h)+H_{\Lambda_{N-1}^{--}}(\sigma) \\
& -\epsilon \sigma_{N-1}^{-}-\frac{1}{2} \xi_{N-1} I_{N-1,-1 / 2}(\sigma)
\end{aligned}
$$

com

$$
\begin{aligned}
& H_{\Lambda_{N-1}^{++}}(\sigma)=-\frac{1}{2} \sum_{n=0}^{N-2} \xi_{n} \sum_{j \in \Lambda_{N-n-2}^{++}} I_{n, j}(\sigma), \\
& H_{\Lambda_{N-1}^{--}}(\sigma)=-\frac{1}{2} \sum_{n=0}^{N-2} \xi_{n} \sum_{j \in \Lambda_{N-n-2}^{--}} I_{n, j}(\sigma),
\end{aligned}
$$

$\mathrm{e}$

$$
\begin{aligned}
H_{\Lambda_{N-1}^{+-}}(\sigma, h)+H_{\Lambda_{N-1}^{-+}}(\sigma, h)= & -\frac{1}{2} \sum_{n=0}^{N-2} \xi_{n} \sum_{j \in \Lambda_{N-n-2}^{+-}} I_{n, j}(\sigma)-\frac{1}{2} \sum_{n=0}^{N-2} \xi_{n} \sum_{j \in \Lambda_{N-n-2}^{-+}} I_{n, j}(\sigma) \\
& -\epsilon\left(\sum_{i \leq 2} \sigma_{N-2, i}^{-}+\cdots+\sum_{i \leq 2^{N-1}}\left(\sigma_{0, i}^{-}+\widetilde{\sigma}_{0, i}^{-}\right)\right) .
\end{aligned}
$$

É possível escrever o fator $I_{N-1,-1 / 2}(\sigma)$ em termos das variáveis de soma e de subtração:

$$
I_{N-1,-1 / 2}(\sigma)=\frac{\left(\sigma_{N-1}^{+}\right)^{2}-\left(\sigma_{N-1}^{-}\right)^{2}}{4}+\sigma_{N-1}^{+}\left(\sigma_{N-1}^{0}+\widetilde{\sigma}_{N-1}^{0}\right)+\sigma_{N-1}^{0} \widetilde{\sigma}_{N-1}^{0},
$$


com $\sigma_{N-1}^{0}$ e $\widetilde{\sigma}_{N-1}^{0}$ correspondendo às variáveis associadas aos blocos admissíveis $\Lambda_{N-1}^{-+}$e $\Lambda_{N-1}^{+-}$.

Pela mesma mudança de variável, decompomos a medida de probabilidade (3.1.4) (nela fazemos $\Lambda=\Lambda_{N}$ ) no produto

$$
\mu_{\Lambda_{N}}(\sigma, h)=\mu_{\Lambda_{N-1}^{++}, \Lambda_{N-1}^{--}}\left(\sigma_{N-1}^{+}, \sigma_{N-1}^{-}\right) \cdot \mu_{\Lambda_{N-1}^{-+}}\left(\sigma_{N-1}^{0}, h\right) \cdot \mu_{\Lambda_{N-1}^{+-}}\left(\widetilde{\sigma}_{N-1}^{0}, h\right)
$$

Definido o modelo, temos condições de buscar um resultado análogo ao do Teorema 3.1.2. Para tanto devemos dispor de uma relação de recorrência para o parâmetro de ordem $m^{2}$ (note que o campo externo utilizado preserva a simetria $\mu_{\Lambda_{N}}(\sigma)=\mu_{\Lambda_{N}}(-\sigma)$, o que nos permite escrever $R_{\mu}$ como $\left.m^{2}\right)$. Partindo da definição (3.1.16) chegamos, como em (3.1.17), a

$$
\begin{aligned}
f_{N}^{0}(N)= & \frac{1}{2^{4 N}} \mu_{\Lambda_{N}}\left[\left(\sigma_{N}^{0}\right)^{2}\right]=\frac{1}{2^{4 N}} \mu_{\Lambda_{N}}\left[\left(\sigma_{N-1}^{+}+\sigma_{N-1}^{0}+\widetilde{\sigma}_{N-1}^{0}\right)^{2}\right] \\
= & \frac{1}{2^{4 N}} \mu_{\Lambda_{N}}\left[\left(\sigma_{N-1}^{0}\right)^{2}+\left(\widetilde{\sigma}_{N-1}^{0}\right)^{2}\right]+\frac{1}{2^{4 N}} \mu_{\Lambda_{N}}\left[\left(\sigma_{N-1}^{+}\right)^{2}\right] \\
& +\frac{1}{2^{4 N-1}} \mu_{\Lambda_{N}}\left[\sigma_{N-1}^{+}\left(\sigma_{N-1}^{0}+\widetilde{\sigma}_{N-1}^{0}\right)+\sigma_{N-1}^{0} \widetilde{\sigma}_{N-1}^{0}\right] .
\end{aligned}
$$

Necessitamos, como no caso sem campo, de estimativas para os termos do membro direito de (3.2.9)

Avaliemos o termo $\mu_{\Lambda_{N}}\left[\left(\sigma_{N-1}^{+}\right)^{2}\right]$ associado aos dois blocos irregulares. Buscamos um limitante inferior livre de campo externo, obtido através da redução do parâmetro $\beta$, o inverso da temperatura (a eliminação do campo, que tende a ordenar alternadamente os spins, é compensada artificialmente por um aumento na temperatura do sistema). Devemos, portanto, verificar para qual $\widetilde{\beta}$ vale a desigualdade

$$
\left\langle\left(\sigma_{N-1}+\sigma_{N-1}^{\prime}\right)^{2}\right\rangle_{\beta, \epsilon} \geq\left\langle\left(\sigma_{N-1}+\sigma_{N-1}^{\prime}\right)^{2}\right\rangle_{\widetilde{\beta}, 0}
$$




$$
\left\langle\left(\sigma_{k}+\sigma_{k}^{\prime}\right)^{2}\right\rangle_{\beta, \epsilon}:=\frac{\sum_{\sigma_{k}, \sigma_{k}^{\prime}=-2^{2 k}}^{2^{2 k}}\left(\sigma_{k}+\sigma_{k}^{\prime}\right)^{2} g\left(\sigma_{k}\right) g\left(\sigma_{k}^{\prime}\right) e^{\frac{\beta \xi_{k}}{2} \sigma_{k} \sigma_{k}^{\prime}+\beta \epsilon\left(\sigma_{k}-\sigma_{k}^{\prime}\right)}}{\sum_{\sigma_{k}, \sigma_{k}^{\prime}=-2^{2 k}}^{2 k} g\left(\sigma_{k}\right) g\left(\sigma_{k}^{\prime}\right) e^{\frac{\beta \xi_{k}}{2} \sigma_{k} \sigma_{k}^{\prime}+\beta \epsilon\left(\sigma_{k}-\sigma_{k}^{\prime}\right)}},
$$

para $k \in\{0, \ldots, N-1\}$. Em (3.2.11), definimos $g\left(\sigma_{k}\right)$ por

$$
g\left(\sigma_{k}\right):=\sum_{\sigma_{k-1}}^{\prime} \prod_{1 \leq i<j \leq 4} e^{\frac{\beta}{2}\left(\sigma_{k-1}(i) \sigma_{k-1}(j)\right)}
$$

(a linha nos diz que as variáveis $\sigma_{k-1}$ satisfazem o vínculo $\sum_{1 \leq i \leq 4} \sigma_{k-1}=\sigma_{k}$ ).

A função $g\left(\sigma_{k}\right)$ satisfaz a identidade

$$
Z_{k}=\sum_{\sigma_{k}=-2^{2 k}}^{2^{2 k}} g\left(\sigma_{k}\right)
$$

$Z_{k}$ a função de partição do $k$-ésimo nível hierárquico.

Para um nível hierárquico $k \in\{1, \ldots, N-1\}$, o limite assintótico $\beta \rightarrow \infty$ de (3.2.11) se escreve como

$$
\begin{aligned}
\left\langle\sigma_{k}+\sigma_{k}^{\prime}\right\rangle_{\beta, \epsilon} & \sim \frac{2^{4(k+1)}\left(g\left(2^{2 k}\right)\right)^{2} e^{\beta \xi_{k} 2^{(4 k-1)}}}{\left(g\left(2^{2 k}\right)\right)^{2} e^{\beta \xi_{k} 2^{(4 k-1)}}\left(1+\frac{1}{2} e^{-\beta \xi_{k} 2^{4 k}+2^{(2 k+1)} \beta|\epsilon|}\right)} \\
& =\frac{2^{4(k+1)}}{1+\frac{1}{2} e^{-\beta \xi_{k} 2^{4 k}+2^{(2 k+1)} \beta|\epsilon|}} .
\end{aligned}
$$

No regime de $\beta$ e $\beta^{\prime}$ grandes (baixas temperaturas, em que há uma fase ferromagnética no modelo sem campo), contribuem para o numerador e o denominador apenas os dois termos marginais $\left(\sigma_{k}, \sigma_{k}^{\prime}=2^{2 k}\right.$ ou $\left.-2^{2 k}\right)$, uma vez que $g\left(\sigma_{k}\right) \leq g\left(2^{2 k}\right)$ e que o campo externo é suficientemente fraco. Busquemos quantificar o "suficientemente fraco". 
Substituindo (3.2.12) em (3.2.10), temos

$$
\frac{2^{4(k+1)}}{1+\frac{1}{2} e^{-\beta \xi_{k} 2^{4 k}+2^{(2 k+1)} \beta|\epsilon|}} \geq \frac{2^{4(k+1)}}{1+\frac{1}{2} e^{-\widetilde{\beta} \xi_{k} 2^{4 k}}},
$$

que é satisfeita se e somente se $\widetilde{\beta} \leq \beta\left(1-\frac{|\epsilon|}{2^{2 k-1} \xi_{k}}\right)$. Como desejamos $\widetilde{\beta}>0$, devemos ter

$$
|\epsilon|<2^{2 k-1} \xi_{k}=2^{[2 k(1-\gamma)-1]} c_{k}(\gamma) \equiv \eta_{k}
$$

dada a definição de $\xi_{k}$. Vemos que para $1<\gamma \leq 2,|\epsilon|$ decresce rapidamente à medida que $k$ cresce. De fato, $\lim _{k \rightarrow \infty} \eta_{k}=0$ para $\widetilde{\beta}>0$, e assim não conseguimos obter um limitante inferior livre de campo não trivial para $\left\langle\left(\sigma_{N-1}+\sigma_{N-1}^{\prime}\right)^{2}\right\rangle_{\beta, \epsilon}$. Como não sabemos estimá-lo por outros meios, somos incapazes de concluir se existe ou não magnetização positiva através deste método.

\subsubsection{Na presença de esparsidade}

Propomos como alternativa ao nosso estudo um campo construído de modo ligeiramente diferente: dizemos que a escala, definida pela seqüência monótona crescente $\mathcal{L}=\left\{L_{n}\right\}_{n \in \mathbb{N}}$, é tal que $L_{n+1}=f_{n}\left(L_{n}\right)$, em que $f_{n}(x)=K^{n} x$, com $K \in \mathbb{N}, K>1$ e $L_{1}=L$ (no caso até então estudado, $f_{n}(x)=f(x)=L \cdot x$; vide Seção 2.3). Definida a nova escala fazemos os blocos irregulares esparsos. Com esparsos queremos dizer que, por exemplo, um bloco $k$-admissível será constituído por dois blocos $(k-1)$ irregulares e por outros $K^{2(k-1)}-2$ blocos $(k-1)$-admissíveis (isto é, o número de blocos irregulares presentes na configuração decai exponencialmente rápido com o aumento da escala) . Provamos a seguir a existência de magnetização positiva para um campo diferente de zero quando empregamos a escala de crescimento exponencial definida acima, para todo $K>1$. Note que esta configuração de campo representa mais realisticamente um padrão aleatório, uma vez que blocos irregulares de nível hierárquico elevado são extremamente raros em uma configuração aleatória típica. 
Comecemos pela extensão das definições do modelo. A distância hierárquica é definida por (3.1.2), com $L_{n}=K^{n-1} L_{n-1}$ e $L_{1}=L$. Analogamente, definimos uma sequência $\Lambda_{n}$ de subconjuntos

$$
\Lambda_{n}:=\left(\left[-L_{n}, L_{n} \cap \mathbb{Z}\right]\right)^{2}, \quad n=1,2, \ldots, N
$$

$\Lambda_{0}:=(-1 / 2,-1 / 2)$ para $n=0$. Dados $n=2, \ldots, N$ e $j \in \Lambda_{N-n}$, definimos em semelhança a (3.1.11) a variável de bloco (para $n=1$ substituímos $K^{n-1}$ por $L$ )

$$
\sigma_{n, j}:=\sum_{i_{1}, i_{2}=0}^{K^{n-1}-1} \sigma_{n-1,\left(K^{n-1} j_{1}+i_{1}, K^{n-1} j_{2}+i_{2}\right)}=\sum_{l, m=0}^{L_{n}-1} \sigma_{L_{n}\left(j_{1}, j_{2}\right)+(l, m)} .
$$

O hamiltoniano para o sistema com campo é expresso por (3.2.1), com $I_{n, j}(\sigma)$ agora definido como

$$
I_{n, j}(\sigma):=\frac{1}{2}\left[\left(\sum_{i_{1}, i_{2}=0}^{K^{n-1}-1} \sigma_{n,\left(K^{n-1} j_{1}+i_{1}, K^{n-1} j_{2}+i_{2}\right)}\right)^{2}-\sum_{i_{1}, i_{2}=0}^{K^{n-1}-1} \sigma_{n,\left(K^{n-1} j_{1}+i_{1}, K^{n-1} j_{2}+i_{2}\right)}^{2}\right]
$$

novamente representando a soma das interações aos pares, sem repetições, dos elementos de um $n$-bloco; $\xi_{n}=c_{n}(\gamma) 2^{-2 \gamma n}$ é tal que

$$
c_{n}(\gamma):= \begin{cases}1 & \text { se } 1 \leq \gamma<2 \\ \log \left(\log L+\frac{n(n+1)}{2} \log K\right) & \text { se } \gamma=2\end{cases}
$$

Supomos que o bloco $\Lambda_{N}, N$-admissível, é contituído pelos blocos $\Lambda_{N-1}^{+}$ $((N-1,+)$-irregular $), \Lambda_{N-1}^{-}\left((N-1,-)\right.$-irregular) e por $\Lambda_{N-1}^{0}$, (soma de $K^{N-1}-2$ blocos $(N-1)$-admissíveis). Definindo as variáveis de soma e subtração dos spins dos blocos irregulares (dois blocos por nível) por (3.2.2) e (3.2.3), respectivamente, podemos decompor (3.2.1) na soma 


$$
\begin{aligned}
H_{\Lambda_{N}}(\sigma, h)= & H_{\Lambda_{N-1}^{0}}(\sigma, h)+H_{\Lambda_{N-1}^{+}}(\sigma)+H_{\Lambda_{N-1}^{-}}(\sigma) \\
& -\epsilon \sigma_{N-1}^{-}-\frac{1}{2} \xi_{N-1} I_{N-1,-1 / 2}(\sigma)
\end{aligned}
$$

com $H_{\Lambda_{N-1}^{+}}(\sigma)$ e $H_{\Lambda_{N-1}^{-}}(\sigma)$ definidos por (3.2.5) e (3.2.6), respectivamente, e

$$
\begin{aligned}
H_{\Lambda_{N-1}^{0}}(\sigma, h)= & -\frac{1}{2} \sum_{n=0}^{N-2} \xi_{n} \sum_{j \in \Lambda_{N-n-2}^{0}} I_{n, j}(\sigma) \\
& -\epsilon\left(\sum_{i \leq 2} \sigma_{N-2, i}^{0} \cdots+\sum_{i \leq L_{N-1}-2}\left(\sigma_{0, i}^{-}+\widetilde{\sigma}_{0, i}^{-}\right)\right) .
\end{aligned}
$$

Reescrevemos $I_{N-1,-1 / 2}(\sigma)$ (em analogia a (3.2.7)) em termos das variáveis de soma e subtração:

$$
I_{N-1,-1 / 2}(\sigma)=\frac{\left(\sigma_{N-1}^{+}\right)^{2}-\left(\sigma_{N-1}^{-}\right)^{2}}{4}+\sigma_{N-1}^{+} \sum_{l=1}^{K^{2(N-1)}-2} \sigma_{N-1, l}^{0}+I_{N-1,-1 / 2}^{0}(\sigma),
$$

$I_{N-1,-1 / 2}^{0}(\sigma)$ referente aos spins dos blocos admissíveis.

A medida de probabilidade (3.2.8) se generaliza como

$$
\mu_{\Lambda_{N}}(\sigma, h)=\mu_{\Lambda_{N-1}^{+}, \Lambda_{N-1}^{-}}\left(\sigma_{N-1}^{+}, \sigma_{N-1}^{-}\right) \prod_{l=1}^{K^{2(N-1)}-2} \mu_{\Lambda_{N-1, l}^{0}}\left(\sigma_{N-1, l}^{0}, h\right)
$$

\section{Relação de recorrência para $m^{2}$}

Devemos, por fim, obter uma relação de recorrência para o parâmetro de ordem $m^{2}$. Partindo da definição (3.1.16) chegamos, como em (3.1.17), a 


$$
\begin{aligned}
f_{N}^{0}(N)= & \frac{1}{L_{N}^{4}} \mu_{\Lambda_{N}}\left[\left(\sigma_{N}^{0}\right)^{2}\right]=\frac{1}{L_{N}^{4}} \mu_{\Lambda_{N}}\left[\left(\sigma_{N-1}^{+}+\sum_{l=1}^{K^{2(N-1)}-2} \sigma_{N-1, l}^{0}\right)^{2}\right] \\
= & \frac{1}{L_{N}^{4}} \mu_{\Lambda_{N}}\left[\left(\sigma_{N-1}^{+}\right)^{2}\right]+\frac{1}{L_{N}^{4}} \mu_{\Lambda_{N}}\left[\sum_{l=1}^{K^{2(N-1)}-2}\left(\sigma_{N-1, l}^{0}\right)^{2}\right] \\
& +\frac{2}{L_{N}^{4}} \mu_{\Lambda_{N}}\left[\sigma_{N-1}^{+} \sum_{l=1}^{K^{2(N-1)}-2} \sigma_{N-1, l}^{0}+I_{N-1,-1 / 2}^{0}(\sigma)\right] .
\end{aligned}
$$

Busquemos estimar (3.2.16) termo a termo. Comecemos provando a desigualdade de Griffiths

$$
\mu_{\Lambda_{N-1}^{0}}\left[\sum_{l=1}^{K^{2(N-1)}-2}\left(\sigma_{N-1, l}^{0}\right)^{2}\right] \geq\left(K^{2(N-1)}-2\right) \mu_{\Lambda_{N-1}^{0}}\left[\left(\sigma_{N-1}^{0}\right)^{2}\right]
$$

Temos, para todo $j \in\left\{1, \ldots, K^{2(N-1)}-2\right\}$, a identidade

$$
\mu_{\Lambda_{N-1}^{0}}\left[\left(\sigma_{N-1, j}^{0}\right)^{2}\right]=\frac{\sum_{\sigma_{N-1, j}^{0}}\left(\sigma_{N-1, j}^{0}\right)^{2} f\left(\sigma_{N-1, j}^{0}\right) F\left(\sigma_{N-1, j}^{0}\right)}{\sum_{\sigma_{N-1, j}^{0}} f\left(\sigma_{N-1, j}^{0}\right) F\left(\sigma_{N-1, j}^{0}\right)},
$$

com

$$
\begin{aligned}
F\left(\sigma_{N-1, j}^{0}\right): & =\sum_{\sigma_{N-1}^{+}} \prod_{\substack{i=1, i \neq j}}^{K_{\sigma_{N-1, i}(N-1)}-2}\left[f\left(\sigma_{N-1}^{+}\right) \prod_{\substack{i=1, i \neq j}}^{K^{2(N-1)}-2} f\left(\sigma_{N-1, i}^{0}\right) \times\right. \\
& \left.\times \cosh \left(\frac{\beta \xi_{N-1}}{2} \sigma_{N-1, j}^{0}\left(\sigma_{N-1}^{+}+\sum_{\substack{i=1, i \neq j}}^{K^{2(N-1)}-2} \sigma_{N-1, i}^{0}\right)\right)\right]
\end{aligned}
$$

uma função par e monótona crescente em $\sigma_{N-1, j}^{0}$, bem como a medida de probabilidade normalizada $f\left(\sigma_{N-1, j}^{0}\right)$. A prova de (3.2.17) se reduz a da desigualdade 


$$
\frac{\sum_{\sigma_{N-1, j}^{0}}\left(\sigma_{N-1, j}^{0}\right)^{2} f\left(\sigma_{N-1, j}^{0}\right) F\left(\sigma_{N-1, j}^{0}\right)}{\sum_{\sigma_{N-1, j}^{0}} f\left(\sigma_{N-1, j}^{0}\right) F\left(\sigma_{N-1, j}^{0}\right)} \geq \sum_{\sigma_{N-1, j}^{0}}\left(\sigma_{N-1, j}^{0}\right)^{2} f\left(\sigma_{N-1, j}^{0}\right)
$$

Note que o membro direito de (3.2.19) nada mais é do que a esperança com respeito à medida $f\left(\sigma_{N-1, j}^{0}\right)$ na ausência de campo. A idéia da prova é a mesma da desigualdade (A.3), fundamental na prova do Lema 3.1.4. De fato, podemos reescrever (3.2.19) como

$$
\begin{aligned}
& \mathbb{E}\left[\left(\sigma_{N-1, j}^{0}\right)^{2} F\left(\sigma_{N-1, j}^{0}\right)\right]-\mathbb{E}\left[\left(\sigma_{N-1, j}^{0}\right)^{2}\right] \mathbb{E}\left[F\left(\sigma_{N-1, j}^{0}\right)\right]= \\
& =\frac{1}{2} \mathbb{E}\left[\left(\left(\sigma_{N-1, j}^{0}\right)^{2}-\left(\bar{\sigma}_{N-1, j}^{0}\right)^{2}\right)\left(F\left(\sigma_{N-1, j}^{0}\right)-F\left(\bar{\sigma}_{N-1, j}^{0}\right)\right)\right] \geq 0,
\end{aligned}
$$

com $\left(\sigma_{N-1, j}^{0}\right)^{2}$ e $\left(\bar{\sigma}_{N-1, j}^{0}\right)^{2}$ duas variáveis aleatórias independentes e identicamente distribuídas.

Apliquemos o Lema 3.1.4 sobre o último termo de (3.2.16). Definindo o vetor $K^{2(N-1)}-2$

$Y$ pela identidade $X:=\left(\sigma_{N-1}^{1}+\sigma_{N-1}^{2}\right) \quad \sum_{l=1} \sigma_{N-1, l}^{0}=1 \cdot Y$, o vetor $a \in \mathbb{R}^{\alpha}$, $\alpha=2\left(K^{2(N-1)}-2\right)$ de componentes $a_{i}=\beta \xi_{N-1} / 2$, e a distribuição de probabilidade

$$
P_{Y}(X)=\sum_{\sigma} \prod_{i=1}^{K^{2(N-1)}-2} \mu_{\Lambda_{N}}(\sigma, h) \delta_{x_{i}, Y_{i}}
$$

obtemos

$\mu_{\Lambda_{N}}\left[\sigma_{N-1}^{+} \sum_{l=1}^{K^{2(N-1)}-2} \sigma_{N-1, l}^{0}\right] \geq 2\left(K^{2(N-1)}-2\right) \bar{\lambda}_{N-1} \lambda_{N-1}^{0} \tanh \left(\frac{\beta \xi_{N-1}}{2} \bar{\lambda}_{N-1} \lambda_{N-1}^{0}\right)$

com as definições

$$
\bar{\lambda}_{N-1}^{2}:=\mathbb{E}\left[\left(\sigma_{N-1}^{1}\right)^{2}\right]=\mathbb{E}\left[\left(\sigma_{N-1}^{2}\right)^{2}\right]
$$


e

$$
\left(\lambda_{N-1}^{0}\right)^{2}:=\mathbb{E}\left[\left(\sigma_{N-1,1}^{0}\right)^{2}\right]=\cdots=\mathbb{E}\left[\left(\sigma_{N-1, \alpha}^{0}\right)^{2}\right]
$$

É simples demonstrar que $\bar{\lambda}_{N-1} \geq \lambda_{N-1}^{0}$. De fato,

$$
\bar{\lambda}_{N-1}^{2}=\frac{\sum_{\sigma} \sigma^{2} f(\sigma) \cosh (\beta \epsilon \sigma)}{\sum_{\sigma} f(\sigma) \cosh (\beta \epsilon \sigma)} \geq \frac{\sum_{\sigma} \sigma^{2} f(\sigma)}{\sum_{\sigma} f(\sigma)}=\left(\lambda_{N-1}^{0}\right)^{2}
$$

já que a medida de probabilidade normalizada $f(\sigma)$ é uma função par (a desigualdade segue em analogia a (3.2.20)). Logo

$\mu_{\Lambda_{N}}\left[\sigma_{N-1}^{+} \sum_{l=1}^{K^{2(N-1)}-2} \sigma_{N-1, l}^{0}\right] \geq 2\left(K^{2(N-1)}-2\right)\left(\lambda_{N-1}^{0}\right)^{2} \tanh \left(\frac{\beta \xi_{N-1}}{2}\left(\lambda_{N-1}^{0}\right)^{2}\right)$

Aplicando o Lema 3.1.4 ao termo $\mu_{\Lambda_{N}}\left[I_{N-1,-1 / 2}(\sigma)\right]$, obtemos (o resultado segue em analogia ao estudo do caso sem campo; os detalhes do cálculo serão omitidos)

$\mu_{\Lambda_{N}}\left[I_{N-1,-1 / 2}^{0}(\sigma)\right] \geq \frac{\left(K^{2(N-1)}-2\right)\left(K^{2(N-1)}-3\right)}{2}\left(\lambda_{N-1}^{0}\right)^{2} \tanh \left(\frac{\beta \xi_{N-1}}{2}\left(\lambda_{N-1}^{0}\right)^{2}\right)$,

uma vez que há em um bloco $N$-admissível $\left(K^{2(N-1)}-2\right)\left(K^{2(N-1)}-3\right) / 2$ pares de blocos $(N-1)$-admissíveis. Por fim, empregamos a desigualdade

$$
\mu_{\Lambda_{N}}\left[\left(\sigma_{N-1}^{+}\right)^{2}\right] \geq 0
$$

uma vez que não temos controle sobre este fator (lembre-se que é justamente este o termo em que alteramos o parâmetro $\beta$ no modelo anterior).

Sejam $x_{N}^{0}(\beta)$ e $x_{N}(\beta)$, respectivamente, as seqüências numéricas associadas às esperanças $f_{N}^{0}(N)$ e $f_{N}(N)$ (esta última sem campo). Definamos a seqüência 


$$
\widetilde{x}_{N}(\beta):=\min \left\{x_{N}^{0}(\beta), x_{N}(\beta)\right\} \text {. }
$$

Desse modo, de (3.2.9), (3.2.17), (3.2.21), (3.2.22) e (3.2.23) concluímos

$$
x_{N}^{0} \geq \frac{K^{2(N-1)}-2}{K^{4(N-1)}} \widetilde{x}_{N-1}\left(1+\left(K^{2(N-1)}+1\right) \tanh \left(\frac{\beta \xi_{N-1}}{2} c_{N-1} L_{N-1}^{4-2 \gamma} \widetilde{x}_{N-1}\right)\right)
$$

(omitimos a dependência de $\widetilde{x}_{N-1}$ com respeito a $\beta$ ).

Dadas a definição (3.2.14) e a identidade

$$
1+\left(K^{2(N-1)}+1\right) \tanh x=\frac{1+\frac{2}{K^{2(N-1)}}-e^{-2 x}}{1+e^{-2 x}},
$$

obtemos de (3.2.24)

$$
x_{N}^{0} \geq \widetilde{x}_{N-1}\left(1-\frac{2}{K^{2(N-1)}}\right)\left(\frac{1+\frac{2}{K^{2(N-1)}}-\alpha_{K}(N-1)\left(\frac{N(N-1)}{2}\right)^{-\beta \widetilde{x}_{N-1}}}{1+\alpha_{K}(N-1)\left(\frac{N(N-1)}{2}\right)^{-\beta \widetilde{x}_{N-1}}}\right),
$$

com $\alpha_{K}(N-1) \equiv(\log K)^{-\beta \widetilde{x}_{N-1}}$. Vale destacar que usamos o fato do membro direito da desigualdade (3.2.24) ser uma função monótona decrescente em $\gamma$ (o que nos permite a substituição $\gamma=2$ ). Omitiremos a prova.

Devemos obter $\widetilde{x}_{1}(\beta)$ como o mínimo entre $x_{1}^{0}(\beta)$ e $x_{1}(\beta)$. Como

$$
x_{1}^{0}(\beta) \equiv x_{1}(\beta, \epsilon)=\frac{e^{3 \beta}+\cosh 2 \beta \epsilon}{e^{3 \beta}+4 \cosh 2 \beta \epsilon+2 e^{-\beta}+e^{-\beta} \cosh 4 \beta \epsilon},
$$

extraímos

$$
\frac{\partial}{\partial \epsilon} x_{1}(\beta, \epsilon)=\frac{\frac{-\beta e^{-\beta}}{2}\left(\sinh 6 \beta \epsilon-\sinh 2 \beta \epsilon+8 e^{3 \beta} \sinh 4 \beta \epsilon+12 e^{4 \beta} \sinh 2 \beta \epsilon\right)}{\left(e^{3 \beta}+4 \cosh 2 \beta \epsilon+2 e^{-\beta}+e^{-\beta} \cosh 4 \beta \epsilon\right)^{2}}
$$


positivo para todo $\epsilon<0$ e negativo para todo $\epsilon>0$. Isso nos mostra que $x_{1}(\beta, \epsilon)$ apresenta um máximo local em $\epsilon=0$; concluímos, portanto, que $\widetilde{x}_{1}(\beta)=x_{1}^{0}(\beta)$ para $\epsilon$ pequeno.

Para uma temperatura suficientemente baixa (limite assintótico $\beta \rightarrow \infty$ ), extraímos de (3.2.26) o resultado

$$
x_{1}^{0}(\beta) \sim\left[1-\frac{e^{4 \beta(\epsilon-1)}}{2}\right]^{-1},
$$

assintótico a zero para todo $\epsilon>1$. Nesta circunstância, o sistema deixa de exibir ordem de longo alcance. Para $\epsilon<1, x_{1}^{0}(\beta)$ é assintótico a um, e como veremos a seguir, o sistema apresenta uma fase ordenada a baixas temperaturas. Há portanto uma competição entre a interação dos spins com o campo e o ferromagnetismo.

Dessa forma, garantimos a existência, para um campo suficientemente pequeno, de um número $\bar{x}$ tal que $x_{1}^{0}(\beta)=x_{1}(\beta, \epsilon) \geq \bar{x}>0$.

É necessário assegurar, em analogia a (3.1.35), a monotonicidade da função

$$
h_{M}(y):=\frac{1+\frac{2}{K^{2(M-1)}}-\left(\frac{M(M-1) \log K}{2}\right)^{-\beta y}}{1+\left(\frac{M(M-1) \log K}{2}\right)^{-\beta y}},
$$

$M \in \mathbb{N}$, o que segue diretamente de (3.1.36) para todo $y \geq 0$.

Mostremos, por fim, que o sistema apresenta magnetização positiva. Nós não nos preocupamos em obter uma estimativa para a temperatura crítica; não obstante, indicamos como obtê-la. Como no caso sem campo $(L=2)$, supomos que

$$
\widetilde{x}_{k} \geq \widetilde{x}_{1} \geq \bar{x}
$$

é válida para $k=1,2, \ldots, N-1$. Aplicando (3.2.25) sucessivamente e substituindo $\widetilde{x}_{k}$ pelo seu limitante inferior $\bar{x}$ obtemos, dada a monotonicidade de $h_{k-1}(y)$, 


$$
\begin{aligned}
x_{N}^{0} & \geq \bar{x} \prod_{i=1}^{N-1}\left(1-\frac{2}{K^{2 i}}\right)\left(\frac{1+\frac{2}{K^{2 i}}-\alpha_{K}(1)\left(\frac{i(i+1)}{2}\right)^{-\beta \bar{x}}}{1+\alpha_{K}(1)\left(\frac{i(i+1)}{2}\right)^{-\beta \bar{x}}}\right) \\
& =\bar{x} \exp \left\{\sum_{i=1}^{N-1} \log \left[\left(1-\frac{2}{K^{2 i}}\right)\left(\frac{1+\frac{2}{K^{2 i}}-\alpha_{K}(1)\left(\frac{i(i+1)}{2}\right)^{-\beta \bar{x}}}{1+\alpha_{K}(1)\left(\frac{i(i+1)}{2}\right)^{-\beta \bar{x}}}\right)\right]\right\} ;
\end{aligned}
$$

o número $x_{N}^{0}$ será positivo caso a série presente no argumento da exponencial em (3.2.28) convirja para todo $N \in \mathbb{N}$. Podemos reescrevê-la como

$$
\begin{aligned}
\sum_{i=1}^{N-1} & {\left[\log \left(1-\frac{2}{K^{2 i}}\right)+\log \left(1+\frac{2}{K^{2 i}}-\alpha_{K}(1)\left(\frac{i(i+1)}{2}\right)^{-\beta \bar{x}}\right)\right.} \\
& \left.-\log \left(1+\alpha_{K}(1)\left(\frac{i(i+1)}{2}\right)^{-\beta \bar{x}}\right)\right] .
\end{aligned}
$$

Estimemos esta expressão termo a termo. Como $\log \left(1-2 / K^{2 i}\right)<0$ para todo $i \in \mathbb{N}$, temos

$$
\sum_{i=1}^{N-1} \log \left(1-\frac{2}{K^{2 i}}\right) \geq \sum_{i=1}^{\infty} \log \left(1-\frac{2}{K^{2 i}}\right)
$$

O próximo passo é usar a desigualdade

$$
\log (1-x) \geq a x+b
$$

para $0 \leq x \leq 2 / K^{2}$ (obtida diretamente da concavidade da função logarítmo). Os coeficientes $a$ e $b$ são determinados a partir das identidades $\log (1)=b=0$ e $\log \left(1-\frac{2}{K^{2}}\right)=\frac{2 a}{K^{2}}$.

Portanto,

$$
\log (1-x) \geq \frac{K^{2}}{2} \log \left(1-\frac{2}{K^{2}}\right) x
$$

o que resulta em 
$\sum_{i=1}^{N-1} \log \left(1-\frac{2}{K^{2 i}}\right) \geq \log \left(1-\frac{2}{K^{2}}\right) \sum_{i=1}^{\infty} K^{-2 i}=\frac{K^{2}}{K^{2}-1} \log \left(1-\frac{2}{K^{2}}\right)$

Temos também que

$$
\log \left(1+\frac{2}{K^{2 i}}-\alpha_{K}(1)\left[\frac{i(i+1)}{2}\right]^{-\beta \bar{x}}\right) \geq \log \left(1-\alpha_{K}(1)\left[\frac{i(i+1)}{2}\right]^{-\beta \bar{x}}\right)
$$

Da desigualdade

$$
\log (1-x) \geq \frac{1}{\alpha_{K}(1)}\left[\log \left(1-\alpha_{K}(1)\right)\right] x
$$

válida para $0<x \leq \alpha_{K}(1)<1$ (novamente usamos a concavidade da função logarítmo), chegamos a

$$
\log \left(1+\frac{2}{K^{2 i}}-\alpha_{K}(1)\left[\frac{i(i+1)}{2}\right]^{-\beta \bar{x}}\right) \geq\left[\log \left(1-\alpha_{K}(1)\right)\right]\left(\frac{i(i+1)}{2}\right)^{-\beta \bar{x}}
$$

Por fim, da desigualdade $\log (1+x) \leq x, x \in \mathbb{R}_{+}$, obtemos

$$
\log \left(1+\alpha_{K}(1)\left[\frac{i(i+1)}{2}\right]^{-\beta \bar{x}}\right) \leq \alpha_{K}(1)\left(\frac{i(i+1)}{2}\right)^{-\beta \bar{x}}
$$

A substituição de todas estas desigualdades em (3.2.28) resulta em

$$
\begin{aligned}
x_{N}^{0} & =\bar{x}\left(1-\frac{2}{K^{2}}\right)^{K^{2} / K^{2}-1} \exp \left\{-\left[\alpha_{K}(1)-\log \left(1-\alpha_{K}(1)\right)\right] \sum_{i=1}^{N-1}\left(\frac{i(i+1)}{2}\right)^{-\beta \bar{x}}\right\} \\
& \geq \bar{x} C^{\prime} \exp \left\{-c \sum_{i=1}^{N-1} \frac{1}{i^{2 \beta \bar{x}}}\right\}
\end{aligned}
$$

com $C^{\prime}>0$ e $c=2^{\beta \bar{x}}[\alpha(1)-\log (1-\alpha(1))]>0$. A série convergirá para todo $N \in \mathbb{N}$ caso $\beta \bar{x}>1$, o que ocorre para $\beta$ suficientemente grande. Obtemos o valor 
de $\beta_{c}$ comparando o segundo membro de (3.2.29) com $\bar{x}$, graças à hipótese indutiva (3.2.27). Desse modo, asseguramos que $x_{N}^{0}>0$ para $\beta$ suficientemente grande.

Todavia, de (3.1.23)

$$
f_{N}^{0}(p) \geq f_{N}^{0}(N) \equiv x_{N}^{0}>\bar{x}
$$

para todo $N \geq p \geq 1$ e todo $\beta$ suficientemente grande, o que, em vista de $m^{2}=\lim _{n \rightarrow \infty} \lim _{N \rightarrow \infty} f_{N}^{0}(n)$, implica em $m^{2}>\bar{x}>0$. Sintetizamos os resultados acima no

Teorema 3.2.1 O modelo hierárquico de Dyson, com campo externo de intensidade fraca e com regiões irregulares exponencialmente esparsas, apresenta magnetização estritamente positiva, de acordo com (3.2.30), para todo $\beta$ suficientemente grande. Existe portanto, pelo critério 3.1.1, ordem de longo alcance. 


\section{Capítulo 4}

\section{Modelo de contornos hierárquicos}

No presente capítulo, estudamos o modelo de contornos hierárquicos ( $\mathrm{MCH})$ na presença de campo externo (empregamos, como usual, o campo quase aleatório) como um gás de contornos (primeira seção). Buscamos novamente provar a existência de OLA para o modelo em duas dimensões (segunda seção). Vale a pena destacar que este modelo é extremamente artificial, e o seu estudo apenas nos permite uma meio alternativo de confirmar os resultados do capítulo anterior. O material a seguir se baseia nos resultados presentes em [3].

\subsection{Procedimento de renormalização}

O MCH pode ser descrito como segue: um bloco de uma dada escala é constituído pela união disjunta de blocos da escala anterior, com volume interno correspondente ao número de pontos em seu interior. Definimos os contornos como as fronteiras desses blocos em cada escala (ou seja, os contornos são hipercubos), independentes entre si (sem interseções). A escala, como vimos, é definida pela seqüência $\mathcal{L}=\left\{L_{n}\right\}_{n \in \mathbb{N}}$, tal que $L_{n+1}=f_{n}\left(L_{n}\right), f: \mathbb{N} \rightarrow \mathbb{N}$ uma função monótona crescente em $n$. Desse modo, temos um bloco da $N$-ésima escala constituído por $\left(L_{N} / L_{N-1}\right)^{d}$ blocos da $N$ - 1-ésima escala.

É interessante notar que os contornos somente podem ocorrer nas posições previamente estabelecidas dos blocos de cada hierarquia. Isto contrasta com os contornos do modelo de Ising, que podem apresentar tanto variação de forma quanto 
de posição na rede.

Considere um hipercubo $\Lambda_{N} \in \mathbb{Z}^{d}$ centrado na origem, com $\left|\Lambda_{N}\right|=L_{N}^{d}$ seu volume. A energia do sistema é definida pela expressão (2.1.1), bem como sua função de partição por (2.1.3). Adotamos condições positivas de fronteira.

Podemos reescrever a expressão (2.1.3) mais apropriadamente (para cada nível hierárquico) se tratarmos o sistema como um gás de contornos.

Seja

$$
\rho_{\gamma}(\beta):=e^{-\beta|\gamma|}
$$

o peso do contorno $\gamma$ (um contorno separa blocos com sinais de spin distintos em um mesmo nível hierárquico).

A função de partição para o $N$-ésimo nível hierárquico, com condições positivas de fronteira para $\Lambda$, pode ser expressa como

$$
Z_{\Lambda_{N}}^{+}(\beta, h)=e^{\left(h, \Lambda_{N}\right)} \sum_{\Gamma} \prod_{\gamma \in \Gamma} \rho_{\gamma}(\beta) e^{-2 \beta\left(h, \Lambda_{N}^{-}(\Gamma)\right)}
$$

a soma em $\Gamma$ com respeito às famílias de contornos compatíveis (ou seja, contornos diferentes são disjuntos e os sinais que eles determinam coincidem); $\Lambda_{N}^{-}(\Gamma)$ corresponde à região determinada por $\Gamma$ com condição de fronteira interna negativa, $h$ o campo externo (substituímos $\epsilon h_{x}$ por $h_{x}$ em (2.1.1)), e $\left(h, \Lambda_{N}\right) \equiv \sum_{x \in \Lambda_{N}} h_{x}$. Obtemos (4.1.1) de (2.1.3), já que

$$
\exp \left\{-\frac{\beta}{2}\left[\sum_{|x-y|=1} \sigma_{x} \sigma_{y}-2 \sum_{x \in \Lambda_{N}} h_{x} \sigma_{x}\right]\right\}=\prod_{\gamma_{N} \in \Gamma} \rho_{\gamma}(\beta) \prod_{x \in \Lambda_{N}} e^{\beta h_{x} \sigma_{x}}
$$

$\mathrm{e}$

$$
\begin{aligned}
\prod_{x \in \Lambda_{N}} e^{\beta h_{x} \sigma_{x}} & =\prod_{x \in \Lambda_{N} \backslash \Lambda_{N}^{-}(\Gamma)} e^{\beta h_{x}} \prod_{x \in \Lambda_{N}^{-}(\Gamma)} e^{-\beta h_{x}} \\
& =\prod_{x \in \Lambda_{N}^{-}(\Gamma)} e^{-2 \beta h_{x}} \prod_{x \in \Lambda_{N}} e^{\beta h_{x}} \\
& =e^{\beta\left(h, \Lambda_{N}\right)} e^{2 \beta\left(h, \Lambda_{N}^{-}(\Gamma)\right)} .
\end{aligned}
$$


Dada a expressão (4.1.1), enunciamos a seguinte proposição.

Proposição 4.1.1 A função de partição (4.1.1) pode ser escrita, por um processo de renormalização, como

$$
Z_{\Lambda_{N}}^{+}(h, \beta)=C_{1} Z_{\Lambda_{N-1}}^{+}\left(h_{1}, \beta_{1}\right)=C_{1} \sum_{\Gamma_{1}} \prod_{\gamma \in \Gamma_{1}} \rho_{\gamma}\left(\beta_{1}\right) e^{-2 \beta_{1}\left(h_{1}, \Lambda_{N-1}^{-}\left(\Gamma_{1}\right)\right)}
$$

com

$$
\begin{gathered}
h_{1, x}=\frac{2}{\beta L^{d-1}} \sum_{y \in L x} \log \left(\frac{1+e^{-\beta\left(1+h_{y} / 2\right)}}{e^{-\beta h_{y} / 2}+e^{-\beta}}\right), \\
\beta_{1}=\beta L^{d-1}
\end{gathered}
$$

$e$

$$
C_{1}=e^{\left(h, \Lambda_{N-1}\right)} \prod_{x \in \Lambda_{N-1}} \prod_{y \in L x}\left(1+e^{-\beta\left(1+h_{y} / 2\right)}\right)
$$

em que $\Gamma_{1}$ denota o conjunto de contornos das hierarquias grandes (isto é, $N>0$ ) e Lx é o bloco indexado por $x\left(L x:=\left\{y \in \mathbb{Z}^{d}: x_{i} \leq y_{i}<x_{i}+L, i \in\{1,2, \ldots, d\}\right\}\right)$.

O procedimento de renormalização consiste em somar as contribuições dos contornos do mais baixo nível hierárquico (o bloco coincide com um sítio da rede), de modo a reescrevê-la em termos dos níveis mais altos restantes. No último passo, reescrevemos a função de partição total como a função de partição de um único nível hierárquico.

Podemos imediatamente somar os contornos da escala zero e obter:

$$
\sum_{\Gamma} \prod_{\gamma \in \Gamma} \rho_{\gamma}(\beta) e^{-2 \beta\left(h, \Lambda_{N}^{-}(\Gamma)\right)}=\sum_{\Gamma_{1}} \prod_{\gamma \in \Gamma_{1}} \rho_{\gamma}(\beta) e^{-2 \beta\left(h+\delta h, \Lambda_{N-1}^{-}\left(\Gamma_{1}\right)\right)}
$$

com $\delta h_{x}$ definido pela função de partição dos pequenos contornos 


$$
e^{-2 \beta(h+\delta h, L x)}:=\left\{\begin{array}{lll}
\prod_{y \in L x}\left(1+e^{-\beta\left(1+h_{y} / 2\right)}\right) & \text { se } & P_{x}^{-}\left(\Gamma_{1}\right)=0 \\
\prod_{y \in L x}\left(e^{-\beta h_{y} / 2}+e^{-\beta}\right) & \text { se } & P_{x}^{-}\left(\Gamma_{1}\right)=1
\end{array},\right.
$$

dada a definição

$$
P_{x}^{-}\left(\Gamma_{1}\right):=\left\{\begin{aligned}
0, & \text { se entre a fronteira e } L x \text { houver um número par de } \\
& \text { contornos } \\
1, & \text { de outra forma }
\end{aligned}\right.
$$

Devemos justificar a expressão (4.1.7). Comecemos com $P_{x}^{-}\left(\Gamma_{1}\right)=0$. A cada ponto $y \in L x$, podemos ou não associar um contorno; caso $\sigma_{y}=1$, não há contorno, o que nos leva a atribuir peso 1 a esta configuração (lembre-se que para os pontos em que $\sigma=1$ não computamos o campo externo); caso $\sigma_{y}=-1$, há a contribuição de um contorno de perímetro um (podemos fazer essa escolha sem afetar os resultados subseqüentes) e a do campo externo, e assim atribuímos o peso $e^{-\beta\left(1+h_{y} / 2\right)}$ a esta configuração.

Já para $P_{x}^{-}\left(\Gamma_{1}\right)=1$, caso $\sigma_{y}=1$, há um contorno e não há contribuição do campo, o que nos leva ao valor de $e^{-\beta}$ como peso da configuração; caso $\sigma_{y}=-1$, não há contorno, porém há contribuição do campo, de tal modo que atribuímos o valor $e^{-\beta h_{y} / 2}$ à configuração.

Podemos ainda reescrever a função de partição de pequenos contornos, dada a definição (4.1.7), como

$$
\begin{aligned}
e^{-2 \beta\left(h+\delta h, \Lambda_{N-1}^{-}\left(\Gamma_{1}\right)\right)} & =\prod_{x \in \Lambda_{N-1}} \prod_{y \in L x}\left(1+e^{-\beta\left(1+h_{y} / 2\right)}\right)\left(\frac{e^{-\beta h_{y} / 2}+e^{-\beta}}{1+e^{-\beta\left(1+h_{y} / 2\right)}}\right)^{P_{x}^{-}\left(\Gamma_{1}\right)} \\
& =C_{1} e^{-\left(h, \Lambda_{N-1}\right)} \exp \left\{\sum_{x \in \Lambda_{N-1}} \sum_{y \in L x} \log \left(\frac{e^{-\beta h_{y} / 2}+e^{-\beta}}{1+e^{-\beta\left(1+h_{y} / 2\right)}}\right)^{P_{x}^{-}\left(\Gamma_{1}\right)}\right\} \\
& =C_{1} e^{-\left(h, \Lambda_{N-1}\right)} e^{-2 \beta_{1}\left(h_{1}, \Lambda_{N-1}^{-}\left(\Gamma_{1}\right)\right)},
\end{aligned}
$$

em que usamos a definição (4.1.5) na penúltima identidade e a definição (4.1.3) na última. Contraíndo os contornos grandes por um fator de escala $L^{d-1}$, ou seja, 


$$
|\gamma| \rightarrow L^{d-1}|\gamma|
$$

(o que justifica (4.1.4)) e substituíndo (4.1.8) e (4.1.9) em (4.1.6), concluímos

$$
e^{\left(h, \Lambda_{N-1}\right)} \sum_{\Gamma} \prod_{\gamma \in \Gamma} \rho_{\gamma}(\beta) e^{-2 \beta\left(h, \Lambda_{N-1}^{-}(\Gamma)\right)}=C_{1} \sum_{\Gamma_{1}} \prod_{\gamma \in \Gamma_{1}} \rho_{\gamma}\left(\beta_{1}\right) e^{-2 \beta_{1}\left(h_{1}, \Lambda_{N-1}^{-}\left(\Gamma_{1}\right)\right)},
$$

exatamente (4.1.2), como desejávamos provar.

Ao repetirmos o procedimento anterior $n$ vezes obtemos as seguintes fórmulas de recorrência:

$$
Z_{\Lambda_{N}}^{+}(h, \beta)=C_{n} Z_{\Lambda_{N-n}}^{+}\left(h_{n}, \beta_{n}\right)=C_{n} \sum_{\Gamma_{n}} \prod_{\gamma \in \Gamma_{n}} \rho_{\gamma}\left(\beta_{n}\right) e^{-2 \beta_{n}\left(h_{n}, \Lambda_{N-n}^{-}\left(\Gamma_{n}\right)\right)}
$$

com

$$
\begin{gathered}
h_{n, x}=\frac{2}{\beta_{n-1} L^{d-1}} \sum_{y \in L x} \log \left(\frac{1+e^{-\beta_{n-1}\left(1+h_{n-1, y} / 2\right)}}{e^{-\beta_{n-1} h_{n-1, y} / 2}+e^{-\beta_{n-1}}}\right), \\
\beta_{n}=\beta_{n-1} L^{d-1}
\end{gathered}
$$

e

$$
C_{n}=C_{n-1} e^{\left(h_{n-1}, \Lambda_{N-n}\right)} \prod_{x \in \Lambda_{N-n}} \prod_{y \in L x}\left(1+e^{-\beta_{n-1}\left(1+h_{n-1, y} / 2\right)}\right)
$$

Definamos a função de 1-ponto

$$
\left\langle\sigma_{0}\right\rangle_{N}^{+}=Z_{\Lambda_{N}}^{+-1}(h, \beta) \sum_{\Gamma} \prod_{\gamma \in \Gamma} \rho_{\gamma}(\beta) e^{-2 \beta\left(h, \Lambda_{N}^{-}(\Gamma)\right)} \sigma_{0}(\Gamma)
$$

com 


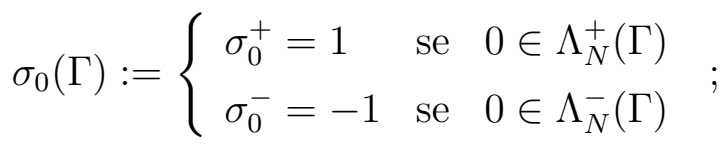

o zero no membro esquerdo de (4.1.11) se refere à origem em $\mathbb{Z}^{d}$ e o zero no membro direito representa o passo de iteração. Obtemos o análogo de (4.1.11) para o $n$-ésimo passo com

$$
\sigma_{n}^{ \pm}=\frac{\sigma_{n-1}^{ \pm}+\sigma_{n-1}^{\mp} e^{-\beta_{n}} e^{\mp \beta_{n} h_{n, 0}}}{1+e^{-\beta_{n}} e^{\mp \beta_{n} h_{n, 0}}} .
$$

Desse modo, $\left\langle\sigma_{0}\right\rangle_{N}^{+}=\sigma_{N}^{+}$. O sistema apresenta ordem de longo alcance (magnetização positiva) caso valha

$$
\alpha_{N}=\sigma_{N}^{+}-\sigma_{N}^{-}>0
$$

$\operatorname{com} \sigma_{N}^{-}$representando a função de um ponto com condição de fronteira negativa.

A próxima seção apresenta os resultados obtidos para o $\mathrm{MCH}$ com campo quase aleatório.

\subsection{Existência de ordem de longo alcance}

Devemos estudar a função $\alpha_{N}=\sigma_{N}^{+}-\sigma_{N}^{-}$para o campo quase aleatório com o intuito de estabelecer a existência de ordem de longo alcance a baixas temperaturas.

\subsubsection{Resultados para um campo externo não esparso}

Comecemos com $d=2$ e $L_{n}=2 L_{n-1}(L=2)$, ou seja, uma escala de crescimento potencial. Sabemos que para esta configuração de campo, cada bloco $n$-admissível é constituido por dois blocos $n-1$-admissíveis, um bloco $(n-1,+)$-irregular e um bloco $(n-1,-)$-irregular.

Neste modelo em particular somos obrigados a definir a origem da rede; em princípio, não privilegiamos nenhum ponto, o que confere à origem um caráter 
aleatório. Desse modo, dada a estrutura do campo, se atribuirmos a mesma probabilidade da origem ocupar qualquer um de seus blocos constituíntes (o que equivale a fixá-la em qualquer ponto da rede), temos para um bloco $N$-admissível

$$
\begin{aligned}
\alpha_{N, r}= & \sigma_{N, r}^{+}-\sigma_{N, r}^{-} \\
= & \frac{1}{2}\left(\frac{\sigma_{N-1, r}^{+}+\sigma_{N-1, r}^{-} e^{-\beta_{N}\left(1+h_{N}\right)}}{1+e^{-\beta_{N}\left(1+h_{N}\right)}}-\frac{\sigma_{N-1, r}^{-}+\sigma_{N-1, r}^{+} e^{-\beta_{N}\left(1-h_{N}\right)}}{1+e^{-\beta_{N}\left(1-h_{N}\right)}}\right) \\
& +\frac{1}{4}\left(\frac{\sigma_{N-1, i r_{+}}^{+}+\sigma_{N-1, i r_{+}}^{-} e^{-\beta_{N}\left(1+h_{N}\right)}}{1+e^{-\beta_{N}\left(1+h_{N}\right)}}-\frac{\sigma_{N-1, i r_{+}}^{-}+\sigma_{N-1, i r_{+}}^{+} e^{-\beta_{N}\left(1-h_{N}\right)}}{1+e^{-\beta_{N}\left(1-h_{N}\right)}}\right) \\
& +\frac{1}{4}\left(\frac{\sigma_{N-1, i r_{-}}^{+}+\sigma_{N-1, i r_{-}}^{-} e^{-\beta_{N}\left(1+h_{N}\right)}}{1+e^{-\beta_{N}\left(1+h_{N}\right)}}-\frac{\sigma_{N-1, i r_{-}}^{-}+\sigma_{N-1, i r_{-}}^{+} e^{-\beta_{N}\left(1-h_{N}\right)}}{1+e^{-\beta_{N}\left(1-h_{N}\right)}}\right) \\
= & \frac{1}{4}\left(2 \alpha_{N-1, r}+\alpha_{N-1, i r_{+}}+\alpha_{N-1, i r_{-}}\right)\left(\frac{1-e^{-\beta_{N}}}{1+e^{-\beta_{N}}}\right),
\end{aligned}
$$

uma vez que

$h_{N}=\frac{2}{\beta_{N-1}}\left[\log \left(\frac{1+e^{-\beta_{N-1}\left(1+h_{N-1,+}\right)}}{e^{-\beta_{N-1} h_{N-1,+}}+e^{-\beta_{N-1}}}\right)-\log \left(\frac{1+e^{-\beta_{N-1}\left(1+h_{N-1,+}\right)}}{e^{-\beta_{N-1} h_{N-1,+}}+e^{-\beta_{N-1}}}\right)\right]=0$

$\left(h_{N-1, r}=0\right.$ e $\left.h_{N-1,+}=-h_{N-1,-}\right)$. Note que a igualdade acima nos permite concluir que, se $h_{1, r}=0$ (o que de fato ocorre), $h_{i, r}=0$ para todo $i \in\{1,2, \ldots, N\}$.

Extraímos da definição de $\alpha_{N, i r_{ \pm}}$a relação de recorrência

$$
\begin{aligned}
& \alpha_{N-1, i r_{ \pm}}=\left(\frac{\sigma_{N-2, i r_{ \pm}}^{+}+\sigma_{N-2, i r_{ \pm}}^{-} e^{-\beta_{N-1}\left(1+h_{N-1, \pm}\right)}}{1+e^{-\beta_{N-1}\left(1+h_{N-1, \pm}\right)}}-\right. \\
&\left.-\frac{\sigma_{N-2, i r_{ \pm}}^{-}+\sigma_{N-2, i r_{ \pm}}^{+} e^{-\beta_{N-1}\left(1-h_{N-1, \pm}\right)}}{1+e^{-\beta_{N-1}\left(1-h_{N-1, \pm}\right)}}\right) \\
&=\alpha_{N-2, i r_{ \pm}} f\left(h_{N-1, \pm}\right)=\alpha_{0} \prod_{i=1}^{N-1} f\left(h_{i, \pm}\right)=2 \prod_{i=1}^{N-1} f\left(h_{i, \pm}\right),
\end{aligned}
$$

em que usamos na última linha o resultado $\alpha_{0}=2$ e a definição

$$
f\left(h_{i, \pm}\right)=\frac{1-e^{-2 \beta_{i}}}{1+e^{-2 \beta_{i}}+2 e^{-\beta_{i}} \cosh \left(\beta_{i} h_{i, \pm}\right)} .
$$


Das expressões (4.2.12) e (4.2.13) obtemos

$$
\alpha_{N, r}=\frac{1}{2}\left(\alpha_{N-1, r}+\prod_{i=1}^{N-1} f\left(h_{i,+}\right)+\prod_{i=1}^{N-1} f\left(h_{i,-}\right)\right)\left(\frac{1-e^{-\beta_{N}}}{1+e^{-\beta_{N}}}\right),
$$

e portanto

$$
\alpha_{N, r}=\frac{1}{2^{N-1}} \prod_{i=1}^{N}\left(\frac{1-e^{-\beta_{i}}}{1+e^{-\beta_{i}}}\right)+\sum_{l=1}^{N-1} \frac{1}{2^{l}} g(N-l) \prod_{i=N+1-l}^{N}\left(\frac{1-e^{-\beta_{i}}}{1+e^{-\beta_{i}}}\right)
$$

onde definimos

$$
g(N-l) \equiv \prod_{j=1}^{N-l} f\left(h_{j,+}\right)+\prod_{j=1}^{N-l} f\left(h_{j,-}\right)
$$

Devemos, portanto, calcular (4.2.15) no limite termodinâmico $(N \rightarrow \infty)$ a fim de concluir se há magnetização positiva na presença de campo. Façamo-lo estudando o regime de baixas temperaturas (limite assintótico de $\beta \rightarrow \infty$ ), para o qual há, na ausência de campo, uma fase ferromagnética. Comecemos com

$$
\begin{aligned}
\prod_{i=a}^{N}\left(\frac{1-e^{-\beta_{i}}}{1+e^{-\beta_{i}}}\right) & =\exp \left\{-\sum_{i=a}^{N}\left(2 e^{-\beta_{i}}+O\left(e^{-3 \beta_{i}}\right)\right)\right\} \\
& \leq \exp \left\{-(N-a)\left(2 e^{-\beta_{N}}+O\left(e^{-3 \beta_{N}}\right)\right)\right\} \sim 1
\end{aligned}
$$

válida para todo $a \in\{1, \ldots, N-1\}$.

Necessitamos do limite assintótico de $g(N-l)$. Para tanto, estudemos o campo efetivo $h_{j, \pm}$ em cada nível hierárquico $j$. Se $h_{j-1,+}>1$, de (4.1.10)

$$
\begin{aligned}
h_{j,+} & =\frac{8}{\beta_{j-1}}\left(\beta_{j-1} h_{j-1,+}+\log \left(\frac{1+e^{-\beta_{j-1}\left(1+h_{j-1,+}\right)}}{1+e^{\beta_{j-1}\left(h_{j-1,+}-1\right)}}\right)\right) \\
& \sim \frac{8}{\beta_{j-1}}\left(\beta_{j-1} h_{j-1,+}-\beta_{j-1}\left(h_{j-1,+}-1\right)\right)=8
\end{aligned}
$$

para $h_{j-1,+}=1$, 


$$
h_{j,+}=\frac{8}{\beta_{j-1}}\left(\beta_{j-1}+\log \left(\frac{1+e^{-2 \beta_{j-1}}}{2}\right)\right) \sim \frac{8}{\beta_{j-1}}\left(\beta_{j-1}-\log 2\right) \sim 8,
$$

e para $h_{j-1,+}<1$

$$
\begin{aligned}
h_{j,+} & \sim \frac{8}{\beta_{j-1}}\left(\beta_{j-1} h_{j-1,+}-2 e^{-\beta_{j-1}} \sinh \left(\beta_{j-1} h_{j-1,+}\right)+O\left(e^{-2 \beta_{j-1}}\right)\right) \\
& \sim 8 h_{j-1,+}
\end{aligned}
$$

Se $h_{j-1,-}<-1$, novamente de (4.1.10),

$$
h_{j,-}=\frac{8}{\beta_{j-1}} \log \left(\frac{1+e^{-\beta_{j-1}\left(1+h_{j-1,-}\right)}}{e^{-\beta_{j-1}}+e^{-\beta_{j-1} h_{j-1,-}}}\right) \sim-8
$$

para $h_{j-1,-}=-1$,

$$
h_{j,-}=\frac{8}{\beta_{j-1}}\left(-\beta_{j-1}+\log \left(\frac{2}{1+e^{-2 \beta_{j-1}}}\right)\right) \sim \frac{8}{\beta_{j-1}}\left(-\beta_{j-1}+\log 2\right) \sim-8,
$$

e para $h_{j-1,-}>-1$,

$$
\begin{aligned}
h_{j,-} & \sim \frac{8}{\beta_{j-1}}\left(\beta_{j-1} h_{j-1,-}-2 e^{-\beta_{j-1}} \sinh \left(\beta_{j-1} h_{j-1,-}\right)+O\left(e^{-2 \beta_{j-1}}\right)\right) \\
& \sim 8 h_{j-1,-}
\end{aligned}
$$

Temos da definição (4.2.14), para $\left|h_{j, \pm}\right|>1$, o resultado assintótico

$$
f\left(h_{j, \pm}\right) \sim e^{-\beta_{i}\left(\left|h_{i, \pm}\right|-1\right)} \sim 0
$$

Portanto, caso $h_{i,+}>1$ e $h_{l,-}<-1$ para algum $i$ ou $l \in\{1, \ldots, N-l\}$, dados (4.2.16) e (4.2.18),

$$
g(N-l)=f\left(h_{i,+}\right) \prod_{\substack{j=1, j \neq i}}^{N-l} f\left(h_{j,+}\right)+f\left(h_{l,-}\right) \prod_{\substack{k=1, k \neq l}}^{N-l} f\left(h_{k,-}\right) \sim 0
$$


Se $h_{j,+}<1$ e $h_{j,-}>-1$ para todo $j \in\{1, \ldots, N-l\}$, novamente de (4.2.14) e $(4.2 .16)$,

$$
\begin{aligned}
g(N-l) & \sim \prod_{j=1}^{N-l}\left(\frac{1}{1+e^{-\beta_{j}\left(1-h_{j,+}\right)}}\right)+\prod_{j=1}^{N-l}\left(\frac{1}{1+e^{-\beta_{j}\left(1-\left|h_{j,-}\right|\right)}}\right) \\
& \sim \exp \left\{-\sum_{j=1}^{N-l} e^{-\beta_{j}\left(1-h_{j,+}\right)}\right\}+\exp \left\{-\sum_{j=1}^{N-l} e^{-\beta_{j}\left(1-\mid h_{j,-}\right)}\right\} \\
& \sim 2 .
\end{aligned}
$$

Vemos de (4.1.10) que a configuração $h_{j,+}=1$ e $h_{j,-}=-1$ para todo $j \in\{1, \ldots, N-l\}$ nunca ocorre.

Verifiquemos, portanto, se há ou não magnetização positiva a baixas temperaturas. Suponha que $\left|h_{j, \pm}\right|<1$ para todo $j \in\{1, \ldots, N-l\}$. De (4.2.15), (4.2.17) e (4.2.20), obtemos

$$
\alpha_{N, r} \sim \frac{1}{2^{N-1}}+2 \sum_{l=1}^{N-1} \frac{1}{2^{l}}=\frac{1}{2^{N-1}}+2-\frac{1}{2^{N-1}} \underset{N \rightarrow \infty}{\longrightarrow} 2 .
$$

Repare que este resultado assintótico é, no limite termodinâmico, equivalente ao resultado do modelo sem campo a baixas temperaturas $\left(\sigma_{N, r}^{+} \sim 1\right.$ e $\left.\sigma_{N, r}^{-} \sim-1\right)$. Todavia, quando tomamos o limite termodinâmico, vemos da condição $\left|h_{j, \pm}\right|<1 \mathrm{e}$ do resultado subseqüente $h_{j, \pm} \sim 8 h_{j-1, \pm}$, válido para todo $j \in\{1, \ldots, N-l\}$, que o campo por sítio inicial igual a zero é o único que satisfaz tais condições (pela relação (4.1.10) um campo inicial diferente de zero atinge, em algum passo do procedimento de renormalização, o valor crítico um).

Por exemplo, caso exista um $j \in\{1, \ldots, N-l\}$ tal que $\left|h_{j, \pm}\right|>1$ (os campos $h_{j,-} 1$ e $h_{j,+}$ são simétricos por construção), obtemos de (4.2.15), (4.2.17) e (4.2.19)

$$
\alpha_{N, r} \sim \frac{1}{2^{N-1}}+\sum_{l=1}^{N-1} 0=\frac{1}{2^{N-1}} \underset{N \rightarrow \infty}{\longrightarrow} 0
$$

Assim, não há magnetização positiva nos sistemas com campo por sítio não nulo. 
Devemos, portanto, estudar uma configuração na qual fazemos os blocos irregulares do campo esparsos (em concordância com o desenvolvimento da Subseção 3.2.2).

\subsubsection{Resultados para um campo externo esparso}

Utilizamos para tal uma escala definida pela mesma seqüência $f_{n}\left(L_{n}\right)=K^{n} L_{n}$, $K \in \mathbb{N}, K>1$ e $L_{1}=L$. Se mais uma vez atribuírmos a mesma probabilidade da origem ocupar qualquer um dos blocos, temos que $\alpha_{N, r}$, em analogia ao que fizemos anteriormente, se exprime como

$$
\begin{aligned}
\alpha_{N, r}= & \left(\frac{K^{2(N-1)}-2}{K^{2(N-1)}} \alpha_{N-1, r}+\frac{1}{K^{2(N-1)}}\left(\alpha_{N-1, i r+}+\alpha_{N-1, i r-}\right)\right)\left(\frac{1-e^{-\beta_{N}}}{1+e^{-\beta_{N}}}\right) \\
\cdots= & 2 \prod_{i=1}^{N-1}\left(1-\frac{2}{K^{2 i}}\right)\left(\frac{1-e^{-\beta_{i}}}{1+e^{-\beta_{i}}}\right)\left(\frac{1-e^{-\beta_{N}}}{1+e^{-\beta_{N}}}\right) \\
& +\sum_{l=2}^{N-2}\left[\frac{2}{K^{2(N-l)}}\left(\prod_{i=N-l+1}^{N} 1-\frac{2}{K^{2 i}}\right) g(N-l) \prod_{j=N-l}^{N} \frac{1-e^{-\beta_{j}}}{1+e^{-\beta_{j}}}\right] \\
& +\frac{2}{K^{2(N-1)}} g(N-1)\left(\frac{1-e^{-\beta_{N}}}{1+e^{-\beta_{N}}}\right)
\end{aligned}
$$

com $g(N-l)$ definido por (4.2.16). Obtemos, ao substituírmos por zero os termos com campo $(\operatorname{com} g(k))$ na expressão acima, a desigualdade

$$
\alpha_{N, r} \geq 2 \prod_{i=1}^{N-1}\left(1-\frac{2}{K^{2 i}}\right)\left(\frac{1-e^{-\beta_{i}}}{1+e^{-\beta_{i}}}\right)\left(\frac{1-e^{-\beta_{N}}}{1+e^{-\beta_{N}}}\right)
$$

Para baixas temperaturas, extraímos de (4.2.17)

$$
\begin{aligned}
\alpha_{N, r} & \geq 2 \prod_{i=1}^{N-1}\left(1-\frac{2}{K^{2 i}}\right)\left(\frac{1-e^{-\beta_{i}}}{1+e^{-\beta_{i}}}\right)\left(\frac{1-e^{-\beta_{N}}}{1+e^{-\beta_{N}}}\right) \sim 2 \prod_{i=1}^{N-1}\left(1-\frac{2}{K^{2 i}}\right) \\
& =2 \exp \left\{\sum_{i=1}^{N-1} \log \left(1-\frac{2}{K^{2 i}}\right)\right\} \geq 2 \exp \left\{\sum_{i=1}^{\infty} \log \left(1-\frac{2}{K^{2 i}}\right)\right\} \\
& \geq 2 \exp \left\{\frac{K^{2}}{K^{2}-1} \log \left(1-\frac{2}{K^{2}}\right)\right\}=2\left(1-\frac{2}{K^{2}}\right)^{\frac{K^{2}}{K^{2}-1}}
\end{aligned}
$$


estritamente positivo para $K>1$ (empregamos na obtenção deste resultado algumas desigualdades utilizadas na Subseção 3.2.2). Sintetizamos os resultados acima no

Teorema 4.2.2 O modelo de contornos hierárquicos, com campo externo de intensidade fraca e com regiões irregulares exponencialmente esparsas, apresenta magnetização estritamente positiva, de acordo com (4.2.21), no regime de baixas temperaturas. Existe portanto, pelo critério 3.1.1, ordem de longo alcance. 


\section{Capítulo 5}

\section{Modelo N-vetorial}

Neste capítulo, provamos a existência de ordem de longo alcance (magnetização espontânea) para o modelo N-vetorial com simetria contínua e sem campo externo (o modelo de Ising é o caso particular $N=1$ ), a partir do surgimento de um condensado (estado de ocupação macroscópica) de momento zero. Em seguida introduzimos o campo quase aleatório e mostramos em quais pontos surgem as dificuldades. Como meio de contorná-las propomos o estudo do modelo N-vetorial hierárquico, obtido a partir da substituição do laplaciano discreto do modelo N-vetorial pelo laplaciano hierárquico. O desenvolvimento feito à seguir é fortemente baseado em [19] (modelo N-vetorial e limite infravermelho), em [23], [6] e em [18] (MHD). Vale ressaltar que existem resultados bem estabelecidos para o estudo de campos aleatórios no limite $N \rightarrow \infty$ (vide [15, 20]).

\subsection{Modelo com interação entre vizinhos mais próximos}

Seja $\Lambda$ uma região hipercúbica $\{-L+1, \ldots, 0, \ldots, L\}^{d}$ em um espaço de $d$ dimensões e $|\Lambda|=(2 L)^{d}$ o volume (número de pontos) de $\Lambda$. Consideremos em cada $x \in \Lambda$ uma variável de $\operatorname{spin} \sigma(x) \in \mathbb{R}^{N}$ sujeita à condição subsidiária

$$
\sigma(x)^{2} \equiv \sum_{i=1}^{N} \sigma_{i}(x)^{2}=1, \quad \sigma(x) \equiv\left(\sigma_{1}(x), \ldots, \sigma_{N}(x)\right)
$$


Isto é, para cada $x=\left(x^{1}, \ldots, x^{d}\right), x \in \mathbb{Z}^{d}$, temos um vetor $\sigma$ de $\mathrm{N}$ componentes na esfera unitária $S_{N-1}$ do espaço euclideano de N dimensões. Seja (- $\Delta$ ) o laplaciano de diferença finita,

$$
(-\Delta \sigma)(x)=2 d \sigma(x)-\sum_{i=1}^{d}\left[\sigma\left(x+e_{i}\right)+\sigma\left(x-e_{i}\right)\right]
$$

em que $e_{i}, i=1, \ldots, d$ são vetores unitários ao longo de cada direção.

O hamiltoniano, com condições periódicas de contorno, é

$$
H_{\Lambda}(\sigma)=\frac{1}{2} \sum_{i=1}^{N} \sum_{|x-y|=1}\left(\sigma_{i}(x)-\sigma_{i}(y)\right)^{2}=\sum_{i=1}^{N}\left(\sigma_{i},-\frac{\Delta}{2} \sigma_{i}\right)
$$

em que $(\cdot, \cdot)$ denota o produto escalar

$$
(f, g) \equiv \sum_{x \in \Lambda} f^{*}(x) g(x)
$$

Para $N=1$ temos o modelo de Ising; para $N=2$ o modelo $\mathrm{XY}$ (ou rotor) clássico; para $N=3$ o modelo de Heisenberg clássico. A regra de soma obtida de (5.1.1) é

$$
\frac{1}{|\Lambda|} \sum_{x} \sum_{i=1}^{N} \sigma_{i}(x)^{2}=\frac{1}{|\Lambda|} \sum_{i=1}^{N}\left(\sigma_{i}, \sigma_{i}\right)=1
$$

Definimos a transformada de Fourier $\hat{f}$ de uma função $f: \Lambda \rightarrow \mathbb{C}$ por

$$
\hat{f}(p)=\frac{1}{\sqrt{|\Lambda|}} \sum_{x \in \Lambda} e^{-i p \cdot x} f(x)
$$

$p \in \Lambda^{*}=\left\{\frac{x \pi}{L}, x \in \Lambda\right\}$ um elemento do espaço dos momentos.

Dadas a equação (5.1.5) e a isometria entre os produtos escalares

$$
\sum_{x \in \Lambda} f^{*}(x) g(x)=\sum_{k \in \Lambda^{*}} \hat{f}^{*}(x) \hat{g}(x)
$$


podemos reescrever a regra de soma (5.1.4) como

$$
\frac{1}{|\Lambda|} \sum_{k \in \Lambda^{*}} \sum_{i=1}^{N} \hat{\sigma}_{i}^{*}(k) \hat{\sigma}_{i}(k)=1
$$

Seja

$$
Z_{\Lambda}(\beta)=\int \prod_{x \in \Lambda} d \sigma(x) \delta\left(|\sigma(x)|^{2}-1\right) e^{-\beta H_{\Lambda}(\sigma)}
$$

a função de partição do modelo. Os valores esperados no estado de Gibbs ao inverso da temperatura $\beta$ se escrevem

$$
\langle F\rangle_{\Lambda}=\frac{1}{Z_{\Lambda}(\beta)} \int \prod_{x \in \Lambda} \delta\left(|\sigma(x)|^{2}-1\right) d \sigma(x) F(\sigma(x)) e^{-\beta H_{\Lambda}(\sigma)}
$$

como o usual. Desse modo, a regra de soma (5.1.6) se torna

$$
\frac{1}{|\Lambda|} \sum_{k \in \Lambda^{*}} \sum_{i=1}^{N}\left\langle\hat{\sigma}_{i}^{*}(k) \hat{\sigma}_{i}(k)\right\rangle_{\Lambda}=1
$$

Podemos reescrever (5.1.7) separando a esperança do quadrado do módulo do modo zero de $\sigma$ do restante da soma;

$$
\frac{1}{|\Lambda|} \sum_{i=1}^{N}\left\langle\hat{\sigma}_{i}^{*}(0) \hat{\sigma}_{i}(0)\right\rangle_{\Lambda}+\frac{1}{|\Lambda|} \sum_{\substack{k \in \Lambda^{*}, k \neq 0}} \sum_{i=1}^{N}\left\langle\hat{\sigma}_{i}^{*}(k) \hat{\sigma}_{i}(k)\right\rangle_{\Lambda}=1
$$

que graças à definição (3.1.9) pode ser identificado como o quadrado da magnetização espontânea:

$$
\begin{aligned}
m^{2}(0) & =\lim _{|\Lambda| \uparrow \mathbb{Z}^{d}}\left[\left\langle\left(\frac{1}{|\Lambda|} \sum_{x \in \Lambda} \sigma(x)\right)^{2}\right\rangle\right] \\
& =\lim _{|\Lambda| \uparrow \mathbb{Z}^{d}} \frac{1}{|\Lambda|} \sum_{i=1}^{N}\left\langle\hat{\sigma}_{i}^{*}(0) \hat{\sigma}_{i}(0)\right\rangle_{\Lambda}
\end{aligned}
$$


Dessa forma, vemos de (5.1.8) que haverá magnetização espontânea caso

$$
m^{2}(0)=1-\lim _{|\Lambda| \uparrow \mathbb{Z}^{d}}\left[\frac{1}{|\Lambda|}\left\langle\sum_{\substack{k \in \Lambda^{*}, k \neq 0}} \hat{\sigma}^{*}(k) \hat{\sigma}(k)\right\rangle\right]>0
$$

ou seja, deve valer o limite

$$
\lim _{|\Lambda| \uparrow \mathbb{Z}^{d}}\left[\frac{1}{|\Lambda|}\left\langle\sum_{\substack{k \in \Lambda^{*}, k \neq 0}} \hat{\sigma}^{*}(k) \hat{\sigma}(k)\right\rangle\right]<1
$$

para $\beta>\bar{\beta}=N I(d)$, com $I(d)$ dado por (5.1.21).

\subsubsection{Dominação gaussiana e limite infravermelho para o modelo sem campo externo}

A fim de obter o limite infravermelho (e conseqüentemente (5.1.10)), devemos dispor da chamada dominação gaussiana (cuja prova se encontra no apêndice B)

$$
Z_{\Lambda}(\eta) \leq Z_{\Lambda}(0)
$$

onde:

1. $\eta$ é uma função

$$
\begin{aligned}
\eta: & \Lambda \rightarrow \mathbb{R}^{N} \\
& x \mapsto \eta(x) \in \mathbb{R}^{N} ;
\end{aligned}
$$

2. $Z_{\Lambda}(\eta)$ é a função de partição do modelo N-vetorial modificado, obtida após a substituição, no hamiltoniano (5.1.3), de $\sigma(x)$ por $\sigma(x)+\eta(x), x \in \Lambda$ :

$$
Z_{\Lambda}(\eta)=\int \prod_{x \in \Lambda} \delta\left(\sigma^{2}(x)-1\right) d \sigma(x) e^{-\beta H_{\Lambda}(\sigma+\eta)}
$$


Já que

$$
\begin{aligned}
H_{\Lambda}(\sigma+\eta) & =\left(\sigma+\eta,-\frac{\Delta}{2}(\sigma+\eta)\right) \\
& =\left(\sigma,-\frac{\Delta}{2} \sigma\right)+(\eta,-\Delta \sigma)+\left(\eta,-\frac{\Delta}{2} \eta\right)
\end{aligned}
$$

podemos reescrever (5.1.11) como

$$
\langle\exp \{\beta(\eta, \Delta \sigma)\}\rangle \exp \left\{\beta\left(\eta, \frac{\Delta}{2} \eta\right)\right\}=\frac{Z_{\Lambda}(\eta)}{Z_{\Lambda}(0)} \leq 1
$$

isto é, vale

$$
\langle\exp \{\beta(\eta, \Delta \sigma)\}\rangle \leq \exp \left\{\beta\left(\eta,-\frac{\Delta}{2} \eta\right)\right\} .
$$

Se em (5.1.14) sustituirmos $\eta$ por $\lambda \eta$ e considerarmos a expansão em série de Taylor em $\lambda$ para os dois membros, obtemos

$$
\begin{aligned}
& \lim _{\lambda \rightarrow 0}[\langle\exp \{\beta(\lambda \eta, \Delta \sigma)\}\rangle-1] \lambda^{-2}= \\
& \quad=\lim _{\lambda \rightarrow 0}\left\{\lambda^{-1} \beta\langle(\eta, \Delta \sigma)\rangle+\frac{\beta^{2}}{2}\langle(\eta, \Delta \sigma)(\eta, \Delta \sigma)\rangle+O(\lambda)\right\}
\end{aligned}
$$

para o membro esquerdo de (5.1.14) e

$$
\begin{aligned}
& \lim _{\lambda \rightarrow 0}\left[\exp \left\{\beta\left(\lambda \eta,-\frac{\Delta}{2}(\lambda \eta)\right)\right\}-1\right] \lambda^{-2}= \\
& =\lim _{\lambda \rightarrow 0}\left\{\beta\left(\eta,-\frac{\Delta}{2} \eta\right)+O(\lambda)\right\}
\end{aligned}
$$

para o membro direito.

Como, por invariância translacional

$$
\langle(\eta, \Delta \sigma)\rangle=\sum_{x \in \Lambda}(-\Delta \eta)(x) \cdot\langle\sigma(x)\rangle=\langle\sigma(0)\rangle \cdot \sum_{x \in \Lambda}(-\Delta \eta)(x)=0
$$


$(\langle\sigma(0)\rangle=0$ devido às condições periódicas ou livres de fronteira), obtemos de $(5.1 .14),(5.1 .15)$ e $(5.1 .16)$

$$
\langle(\eta,-\Delta \sigma)(\eta,-\Delta \sigma)\rangle \leq \frac{1}{\beta}(\eta,-\Delta \eta)
$$

Tomando $\eta(x)=\left(\eta_{1}(x), \ldots, \eta_{N}(x)\right)$ como

$$
\eta_{j}(x)=\frac{1}{\sqrt{2}}\left(e^{i k \cdot x}+e^{-i k \cdot x}\right) \delta_{i, j} \quad j=1, \ldots, N
$$

chegamos a

$$
\left\langle\hat{\sigma}^{*}(k) \hat{\sigma}(k)\right\rangle \leq \frac{N}{\beta \omega(k)} \quad k \neq 0
$$

o chamado limite infravermelho, com $\omega(k)$ satisfazendo a equação de autovalor

$$
(-\widehat{\Delta \sigma})(p)=\omega(p) \hat{\sigma}(p), \quad \omega(p)=2 \sum_{i=1}^{d}\left(1-\cos p_{i}\right)
$$

Derivemos explicitamente este último resultado. Escrevendo a transformada de Fourier de (5.1.18) como

$$
\hat{\eta}_{j}(p)=\frac{1}{\sqrt{|\Lambda|}} \sum_{x \in \Lambda} \frac{1}{\sqrt{2}}\left(e^{i(k-p) \cdot x}+e^{-i(k+p) \cdot x}\right) \delta_{i, j}=\sqrt{\frac{|\Lambda|}{2}}\left(\delta_{k,-p}+\delta_{k, p}\right) \delta_{i, j}
$$

obtemos

$$
\begin{aligned}
(\eta,-\Delta \sigma) & =\sum_{p} \hat{\eta}(p)(-\widehat{\Delta \sigma})(p) \\
& =\sum_{p} \omega(p) \hat{\eta}(p) \hat{\sigma}(p) \\
& =\sqrt{\frac{|\Lambda|}{2}} \omega(k)\left(\hat{\sigma}_{i}(k)+\hat{\sigma}_{i}(-k)\right) .
\end{aligned}
$$


Analogamente

$$
\begin{aligned}
\langle(\eta,-\Delta \sigma)(\eta,-\Delta \sigma)\rangle & =\frac{|\Lambda|}{2} \omega^{2}(k)\left\langle\left(\hat{\sigma}_{i}(k)+\hat{\sigma}_{i}(-k)\right)\left(\hat{\sigma}_{i}(k)+\hat{\sigma}_{i}(-k)\right)\right\rangle \\
& =|\Lambda| \omega^{2}(k)\left\langle\hat{\sigma}_{i}(k) \hat{\sigma}_{i}^{*}(k)\right\rangle
\end{aligned}
$$

uma vez que $\left\langle\hat{\sigma}_{i}^{2}(k)\right\rangle$ e $\left\langle\hat{\sigma}_{i}^{2}(-k)\right\rangle$ se anulam (a medida de integração depende unicamente do módulo de $\sigma$; funções que apresentam fases têm valor médio nulo). Como $\sigma(k)$ é uma função real, vale $\hat{\sigma}^{*}(k)=\hat{\sigma}(-k)$.

Temos também que

$$
\begin{aligned}
(\eta,-\Delta \eta) & =\sum_{j=1}^{N} \sum_{p \in \Lambda^{*}} \omega(p)\left|\hat{\eta}_{j}(p)\right|^{2} \\
& =\frac{|\Lambda|}{2} \sum_{p \in \Lambda^{*}} \omega(p)\left(\delta_{k, p}+\delta_{-k, p}\right) \\
& =\frac{|\Lambda|}{2}(\omega(k)+\omega(-k)) \\
& =|\Lambda| \omega(k) .
\end{aligned}
$$

Finalmente, a substituição desses resultados em (5.1.17) nos leva precisamente a (5.1.19).

Verifiquemos agora para quais condições (5.1.10) é válida. Combinando a regra de soma e o limite infravermelho obtemos, no limite termodinâmico,

$$
\begin{aligned}
m^{2}(0) & \geq 1-\lim _{|\Lambda| \uparrow \mathbb{Z}^{d}}\left[\frac{1}{|\Lambda|}\left\langle\sum_{\substack{k \in \Lambda^{*}, k \neq 0}} \frac{N}{\beta \omega(k)}\right\rangle\right] \\
& =1-\frac{N}{\beta} I(d)
\end{aligned}
$$

com

$$
I(d):=\frac{1}{(2 \pi)^{d}} \int_{|k| \leq \pi} \frac{d^{d} k}{\omega(k)} .
$$

Devemos analisar quando $I(d)$ é integrável. O integrando é singular em $k=0$, já que $O(\omega(k))=O\left(k^{2}\right)$ quando $k \rightarrow 0$. Assim sendo, para todo $\delta>0$ 


$$
I(d)=\frac{1}{(2 \pi)^{d}} \int_{|k| \leq \delta} \frac{d^{d} k}{k^{2}}+I_{R}^{\delta}(d)<\infty
$$

em que $I_{R}^{\delta}(d)$ é a parte regular de $I(d)$; a primeira integral do membro direito converge se e somente se $d \geq 3$.

Logo, se $\beta>\bar{\beta} \equiv N I(d)$ e $d \geq 3$ segue (5.1.10), e portanto o modelo N-vetorial exibe ordem de longo alcance.

Na próxima subseção, buscamos provar a existência de longo alcance para o modelo com campo quase aleatório através das técnicas desenvolvidas nesta subseção.

\subsubsection{Extensão do método do limite infravermelho para o campo alternado}

Consideremos o mesmo modelo N-vetorial estudado na seção anterior, com o hamiltoniano

$$
H(\sigma, h)=\left(\sigma,-\frac{\Delta}{2} \sigma\right)+(\sigma, h)
$$

na presença de um campo externo $h$. Estudemos em primeiro lugar o caso alternado. Definimos a nova média térmica, em analogia a (B.1), como

$$
\langle\cdot\rangle_{H}=\frac{\left\langle\cdot e^{-\beta H}\right\rangle}{\left\langle e^{-\beta H}\right\rangle},
$$

com $H$ dado por (5.1.22).

Novamente, a existência de ordem de longo alcance está diretamente associada à existência do limite infravermelho. Para mostrar que tal limite existe é necessário provar o resultado de dominação gaussiana para o novo hamiltoniano:

$$
Z_{\Lambda}(\eta, h) \leq Z_{\Lambda}(0, h),
$$

a perturbação $\eta$ definida por (5.1.12) e 


$$
Z_{\Lambda}(\eta, h):=\int \prod_{x \in \Lambda} \delta\left(|\sigma(x)|^{2}-1\right) d \sigma(x) e^{-\beta H(\sigma+\eta, h)}
$$

A prova de (5.1.24) é semelhante a do caso sem campo: se aplicarmos a Proposição B.3 a $Z_{\Lambda}(\eta(x), h(x))\left(\right.$ definimos neste momento $\left.G_{x}(\sigma):=F(\sigma-\eta(x)) / F(\sigma)\right)$ obtemos

$$
\begin{aligned}
Z_{\Lambda}(\eta, h) & =\int\left\{\prod_{x \in \Lambda} F(\sigma(x)) d \sigma(x)\right\} e^{-\beta H(\sigma+\eta, h)} \\
& =\int\left\{\prod_{x \in \Lambda} G_{x}(\sigma(x)) F(\sigma(x)) d \sigma(x)\right\} e^{-\beta H(\sigma, h)} \\
& =Z_{\Lambda}(0, h)\left\langle\prod_{x \in \Lambda} G_{x}(\sigma(x))\right\rangle_{H} \leq Z_{\Lambda}(0, h) \prod_{x \in \Lambda}\left\langle\prod_{y \in \Lambda} G_{x}(\sigma(y))\right\rangle_{H}^{\frac{1}{\mid \Lambda}} \\
& \leq Z_{\Lambda}(0, h) .
\end{aligned}
$$

Verifiquemos que o hamiltoniano é de fato invariante pela adição da perturbação homogênea $\bar{\eta}$. Da definição (5.1.22) temos

$$
\begin{aligned}
H(\sigma+\bar{\eta}, h) & =\sum_{i=1}^{N}\left\{\left(\sigma_{i}+\bar{\eta}_{i},-\frac{\Delta}{2}\left(\sigma_{i}+\bar{\eta}_{i}\right)\right)+\left(\sigma_{i}+\bar{\eta}_{i}, h_{i}\right)\right\} \\
& =H(\sigma, h)+\sum_{i=1}^{N}\left\{\left(\bar{\eta}_{i},-\frac{\Delta}{2} \bar{\eta}_{i}\right)+\left(\sigma_{i},-\Delta \bar{\eta}_{i}\right)+\left(\bar{\eta}_{i}, h_{i}\right)\right\} \\
& =H(\sigma, h)
\end{aligned}
$$

graças à igualdade $\langle\bar{\eta}, \Delta \sigma\rangle=\langle\Delta \bar{\eta}, \sigma\rangle$ (o laplaciano é um operador auto-adjunto) e ao fato do produto escalar $(\bar{\eta}, h)$ se anular (o campo alternado tem média zero).

Note que, na penúltima passagem de (5.1.26), utilizamos a positividade por reflexão de $\langle\cdot\rangle_{H}$ com respeito ao morfismo $\theta^{\prime}=-\theta$, válida para o caso em que o campo é alternado. De fato, podemos exprimir o hamiltoniano (5.1.22) como $-H=A+\theta^{\prime} A+B+\theta^{\prime} B+\sum_{i=1}^{k} C_{i} \theta^{\prime} C_{i}$ (vide Teorema B.1, Apêndice B); a interação dos spins com o campo pode ser escrita como 


$$
\sum_{x \in \Lambda} h_{x} \sigma_{x}=\sum_{x \in \Lambda_{+}} h_{x} \sigma_{x}-\sum_{\theta^{\prime} x \in \Lambda_{-}} h_{\theta^{\prime} x} \sigma_{\theta^{\prime} x}=B+\theta^{\prime} B
$$

os elementos de $\Lambda_{+}$mapeados nos elementos de $\Lambda_{-}$por $\theta^{\prime}$ (dada a alternância do campo, $\left.h_{x}=-h_{\theta^{\prime} x}\right)$.

Provamos assim a dominação gaussiana para o modelo com campo alternado. Nosso próximo passo é utilizá-la na obtenção do limite infravermelho. Reescrevemos (5.1.24) como

$$
\begin{aligned}
\langle\exp \{\beta(\eta, \Delta \sigma)\}\rangle_{H} & =\frac{\int \prod_{x \in \Lambda} d \mu(\sigma(x)) e^{-\beta H(\sigma, h)} e^{\beta(\eta, \Delta \sigma)}}{Z_{\Lambda}(0, h)} \\
& \left.=\exp \left\{\beta\left(\eta,-\frac{\Delta}{2} \eta+h\right)\right)\right\} \frac{Z_{\Lambda}(\eta, h)}{Z_{\Lambda}(0, h)} \\
& \leq \exp \left\{\beta\left(\eta,-\frac{\Delta}{2} \eta\right)+\beta(\eta, h)\right\}
\end{aligned}
$$

Analisemos o produto escalar $(h, \eta)$. Se tomarmos $\eta_{k}(x)=e^{i k x}, k \in \Lambda^{*}$ (também um autovetor de (5.1.3)), temos que

$$
(\eta, h)=N \sum_{x \in \Lambda} h_{x} e^{i k x}=N \prod_{j=1}^{d} \sum_{x_{j} \in \bar{\Lambda}} h_{x_{j}} e^{i k_{j} x_{j}}
$$

$\operatorname{com} \bar{\Lambda}=\{-L+1, \ldots, L\}$ e $h_{x} \equiv \prod h_{x_{j}}$ o campo alternado. Caso adotemos $h_{2 x_{j}}=$ $\epsilon^{1 / d}$ e $h_{2 x_{j}+1}=-\epsilon^{1 / d}$ para todo $j=1, \ldots, d$ (a situação com os sinais invertidos não traz nenhuma mudança), vemos da definição acima que $h_{x}$ é alternado. Concluímos de (5.1.28) que as somas presentes em seu membro direito são equivalentes, e que portanto basta analisarmos uma delas. Obtemos

$$
\begin{aligned}
\sum_{x_{j}=-L+1}^{L} h_{x_{j}} e^{i k_{j} x_{j}} & =\epsilon^{1 / d} \sum_{y=-M+1}^{M} e^{2 i k y}-\epsilon^{1 / d} \sum_{y=-M}^{M-1} e^{i k(2 y+1)} \\
& =\epsilon^{1 / d} \frac{e^{2 i k(-M+1)}-e^{2 i k(M+1)}}{1-e^{2 i k}}-\epsilon^{1 / d} e^{i k} \frac{\left(e^{-2 i k M}-e^{2 i k M}\right)}{1-e^{2 i k}} \\
& =-\epsilon^{1 / d} \frac{\sin (2 k M)}{\cos (k / 2)}=0
\end{aligned}
$$


para todo $k=\frac{n \pi}{L} \neq \pi, n \in\{-L+1, \ldots, L-1\}$, com $L=2 M$. Para $k=\pi$ há uma indeterminação (o numerador e o denominador se anulam), de modo que

$$
\epsilon^{1 / d} \lim _{n \rightarrow L} \frac{\sin (n \pi)}{\cos (n \pi / 2 L)}=\epsilon^{1 / d} \lim _{n \rightarrow L} \frac{2 L \cos (n \pi)}{\sin (n \pi / 2 L)}=2 L \epsilon^{1 / d}(-1)^{L}=\epsilon^{1 / d}|\bar{\Lambda}|
$$

O resultado é esperado, uma vez que o modo $k=\pi$, autovetor de $-\Delta$, corresponde à configuração em que os spins são alternados. Note que os demais modos são ortogonais ao campo. Temos, de (5.1.28) e do resultado acima, que

$$
(h, \eta)=N \prod_{j=1}^{d} \epsilon^{1 / d}|\bar{\Lambda}|=N \epsilon|\Lambda| .
$$

Se substituirmos $\eta$ por $\lambda \eta$ em (5.1.27) e efetuarmos sua expansão em série de Taylor, obtemos

$$
\begin{aligned}
& \lim _{\lambda \rightarrow 0}\left[\langle\exp \{\beta(\lambda \eta, \Delta \sigma)\}\rangle_{H}-1\right] \lambda^{-2} \\
& =\lim _{\lambda \rightarrow 0}\left\{\lambda^{-1} \beta\langle(\eta, \Delta \sigma)\rangle_{H}+\frac{\beta^{2}}{2}\langle(\eta, \Delta \sigma)(\eta, \Delta \sigma)\rangle_{H}+O(\lambda)\right\}
\end{aligned}
$$

para o membro esquerdo de (5.1.27) e

$$
\begin{aligned}
& \lim _{\lambda \rightarrow 0}\left[\exp \left\{\beta\left(\lambda \eta,-\lambda \frac{\Delta}{2} \eta+h\right)\right\}-1\right] \lambda^{-2}= \\
& =\lim _{\lambda \rightarrow 0}\left\{\beta\left(\eta,-\frac{\Delta}{2} \eta\right)+\beta \lambda^{-1}(\eta, h)+\frac{\beta^{2}}{2}(\eta, h)^{2}+O(\lambda)\right\}
\end{aligned}
$$

para o membro direito. No entanto, o limite infravermelho somente é obtido da dominação gaussiana quando a esperança $\langle(\sigma,-\Delta \eta)\rangle_{H}=0$ se anula. Temos explicitamente

$$
\begin{aligned}
\langle(\sigma,-\Delta \eta)\rangle_{H} & =\omega(k) \sqrt{|\Lambda|}\langle\widehat{\sigma}(k)\rangle_{H} \\
& =\frac{\omega(k) \sqrt{|\Lambda|}}{Z_{\Lambda}(0, h)} \int \widehat{\sigma}(k)\left\{\prod_{x \in \Lambda} \delta\left((\sigma(x))^{2}-1\right) d \sigma(x)\right\} e^{-\beta H(\sigma, h)}
\end{aligned}
$$


O hamiltoniano (5.1.22) é, na base de Fourier,

$$
H(\sigma, h)=\sum_{\substack{k \in \Lambda^{*}, k \neq \pi}} \frac{\omega(k)}{2} \widehat{\sigma}(k) \widehat{\sigma}^{*}(k)+\frac{\omega(\pi)}{2} \widehat{\sigma}^{2}(\pi)+\epsilon \sqrt{|\Lambda|} \widehat{\sigma}(\pi)
$$

uma vez que

$$
(\sigma, h)=\epsilon \sum_{x \in \Lambda} \sigma(x) e^{-i \pi x}=\epsilon \sqrt{|\Lambda|} \widehat{\sigma}(\pi)
$$

dada a definição (5.1.5). Assim,

$$
\int \widehat{\sigma}(k)\left\{\prod_{x \in \Lambda} \delta\left((\sigma(x))^{2}-1\right) d \sigma(x)\right\} e^{-\beta H(\sigma, h)}=0
$$

para $k \neq \pi$ (note que a medida é invariante pelas trocas simultâneas $\sigma(x) \rightarrow-\sigma(x)$ e $h(x) \rightarrow-h(x)$ para todo $x \in \Lambda)$, e portanto concluímos que $\langle(\sigma,-\Delta \eta)\rangle_{H}=0$.

Como $\left(\eta_{k}, h\right)=N \epsilon \delta_{k, \pi}|\Lambda|$, obtemos de (5.1.27), (5.1.29) e (5.1.30)

$$
\langle(\eta,-\Delta \sigma)(\eta,-\Delta \sigma)\rangle_{H} \leq \frac{1}{\beta}(\eta,-\Delta \eta)
$$

válida para todo $k \neq \pi$, exatamente o limite infravermelho obtido para o caso sem campo.

Para $k=\pi$, substituímos $\eta$ por $\lambda \eta$ em (5.1.27) e efetuamos sua expansão em primeira ordem de $\lambda$, de tal modo que

$$
\left\langle\left(\eta_{\pi}, \Delta \sigma\right)\right\rangle_{H}=-\omega(\pi) \sqrt{|\Lambda|}\langle\widehat{\sigma}(\pi)\rangle_{H} \leq N \epsilon|\Lambda|
$$

Temos, portanto, a desigualdade

$$
\langle\widehat{\sigma}(\pi)\rangle_{H} \leq-\frac{N \epsilon \sqrt{|\Lambda|}}{\omega(\pi)}
$$

Entretanto, necessitamos de um limitante superior para $\left\langle(\widehat{\sigma}(\pi))^{2}\right\rangle_{H}$. Para tanto, recordemo-nos da definição de variância de uma grandeza estatística: 


$$
\operatorname{Var}(\widehat{\sigma}(k)):=\left\langle\left(\widehat{\sigma}(k)-\langle\widehat{\sigma}(k)\rangle_{H}\right)^{2}\right\rangle_{H}=\left\langle(\widehat{\sigma}(k))^{2}\right\rangle_{H}-\langle\widehat{\sigma}(k)\rangle_{H}^{2}
$$

Derivamos de (5.1.32) e (5.1.33) a desigualdade

$$
\left\langle(\widehat{\sigma}(k))^{2}\right\rangle_{H} \leq \operatorname{Var}(\widehat{\sigma}(k))+\frac{N^{2} \epsilon^{2}|\Lambda|}{\omega^{2}(\pi)},
$$

que combinada à regra de soma e ao limite infravermelho fornece

$$
m^{2}(h) \geq 1-\lim _{|\Lambda| \uparrow \mathbb{Z}^{d}}\left[\frac{1}{|\Lambda|}\left\langle\sum_{\substack{k \in \Lambda^{*}, \pi \\ k \neq 0, \pi}} \frac{N}{\beta \omega(k)}\right\rangle\right]-\frac{N^{2} \epsilon^{2}}{\omega^{2}(\pi)}-\frac{\operatorname{Var}(\widehat{\sigma}(k))}{|\Lambda|}
$$

Suponhamos que $\operatorname{Var}(\widehat{\sigma}(k))=o(|\Lambda|)$. Desse modo, (5.1.34) se torna, no limite termodinâmico,

$$
m^{2}(h) \geq 1-\frac{N I(d)}{\beta}-\left(\frac{N \epsilon}{4 d}\right)^{2}
$$

estritamente positivo para $d \geq 3$ e para $\beta \geq \bar{\beta} \equiv N I(d) /\left(1-\left(\frac{N \epsilon}{4 d}\right)^{2}\right)$, com $\bar{\beta}>0$ caso $\epsilon<\frac{4 d}{N}(\omega(\pi)=4 d)$. Desse modo, o modelo N-vetorial com campo alternado apresenta ordem de longo alcance para $d \geq 3$ e $\beta>\bar{\beta}$.

É interessante notar que a ocupação macroscópica do modo zero (o que corresponde ao quadrado da magnetização ser estritamente positivo) somente ocorre se a intensidade do campo externo $\epsilon$ for suficientemente fraca, uma vez que o modo $k=\pi$ também é ocupado macroscopicamente. Vemos, portanto, uma competição entre a interação ferromagnética dos spins (que tende a alinhá-los entre si) e a interação dos spins com o campo (que tende a alinhá-los alternadamente).

Poderíamos questionar se a variância do modo $k=\pi$ é, de fato, de ordem inferior ao volume do sistema, como assumimos acima. No entanto, é simples verificar que isso ocorre para o caso gaussiano (as componentes do N-vetor $\sigma(x)$ são independentes entre si). A média térmica $\langle\widehat{\sigma}(\pi)\rangle_{H}$ é dada por 


$$
\begin{aligned}
\langle\widehat{\sigma}(\pi)\rangle_{H} & =\frac{\int\left(\widehat{\sigma}(\pi)-\frac{\epsilon \sqrt{|\Lambda|}}{\omega(\pi)}\right)\left\{\prod_{x \in \Lambda} d \sigma(x)\right\} \exp \left\{-\beta \sum_{k \in \Lambda^{*}} \frac{\omega(k)}{2} \widehat{\sigma}(k) \widehat{\sigma}(k)^{*}\right\}}{Z_{\Lambda}(0, h)} \\
& =-\frac{\epsilon \sqrt{|\Lambda|}}{\omega(\pi)} .
\end{aligned}
$$

Já a variância $\operatorname{Var}(\widehat{\sigma}(\pi))$ se expressa como

$$
\begin{aligned}
& \int M(\widehat{\sigma}(\pi))\left\{\prod_{x \in \Lambda} d \sigma(x)\right\} \exp \left\{-\beta \sum_{\substack{k \in \Lambda^{*}, k \neq \pi}} \frac{\omega(k)}{2} \widehat{\sigma}(k) \widehat{\sigma}(k)^{*}-\frac{\omega(\pi)}{2} M(\widehat{\sigma}(\pi))\right\} \\
& Z_{\Lambda}(0, h)
\end{aligned}
$$

o que concorda com a nossa suposição (definimos na expressão acima a função $\left.M(\widehat{\sigma}(\pi))=\left(\widehat{\sigma}(\pi)-\langle\widehat{\sigma}(\pi)\rangle_{H}\right)^{2}\right)$.

Para o caso do campo quase aleatório, somos incapazes de demonstrar a positividade por reflexão de $\langle\cdot\rangle_{H}$, e dessa forma o método deixa de ser aplicável.

Propomos então o estudo do modelo N-vetorial hierárquico como uma alternativa para a resolução do problema com campo quase aleatório. Este é o assunto abordado na próxima seção.

\subsection{Modelo com interação hierárquica}

Nesta seção, introduzimos o laplaciano hierárquico e provamos a existência de ordem de longo alcance no modelo N-vetorial através do mesmo fenômeno de condensação (ocupação macroscópica) do estado fundamental (que no caso será representado pela autofunção do último nível hierárquico presente no sistema finito; os detalhes serão exibidos a seguir), tanto para o caso sem campo (Subseção 5.2.2) como para o caso com campo (Subseção 5.2.3). Os resultados referentes ao teorema espectral para o laplaciano hierárquico seguem de [18]. 


\subsubsection{Laplaciano hierárquico}

Dados números inteiros $L, K>1$ e $d \geq 1$, seja

$$
\Lambda_{K}=\left\{0,1, \ldots, L^{K}-1\right\} \subset \mathbb{Z}^{d}
$$

um hipercubo de cardinalidade $\left|\Lambda_{K}\right|=L^{d K}=n$ ( $L$ é o comprimento do bloco hierárquico fundamental, $d$ é a dimensão do hipercubo e $K$ representa o último nível hierárquico do sistema). Como qualquer ponto $l \in \Lambda_{K}$ pode ser escrito em uma base $L^{d}$-ádica

$$
l=\sum_{k=1}^{K} \theta_{k} L^{k-1}, \quad \theta_{k} \in\{0,1, \ldots, L-1\}^{d}
$$

usamos $\theta=\left(\theta_{1}, \ldots, \theta_{K}\right)$ para representar pontos em $\Lambda_{K}$ e indexar componentes de um vetor $u=\left(u_{\theta}\right)$ em $\mathbb{R}^{\Lambda_{K}}$.

Definimos $B: \mathbb{R}^{\Lambda_{K}} \longrightarrow \mathbb{R}^{\Lambda_{K-1}}$ como o operador de bloco

$$
(B u)_{\tau}=\frac{1}{L^{d / 2}} \sum_{\theta_{1} \in\{0,1, \ldots, L-1\}^{d}} u_{\left(\theta_{1}, \tau\right)}
$$

seja $B^{*}: \mathbb{R}^{\Lambda_{K-1}} \longrightarrow \mathbb{R}^{\Lambda_{K}}$

$$
\left(B^{*} v, u\right)_{\Lambda_{K}}=(v, B u)_{\Lambda_{K-1}}
$$

seu adjunto com respeito ao produto interno $(u, v)_{\Lambda_{K}}=\sum_{\theta} u_{\theta} v_{\theta}$, dado por

$$
\left(B^{*} v\right)_{\left(\theta_{1}, \tau\right)}=\frac{1}{L^{d / 2}} v_{\tau}
$$

Note que a operação $(B u)_{\tau}$ consiste em somar as componentes de $u$ do primeiro bloco hierárquico indexado por $\tau$, componentes estas representadas por $u_{\left(\theta_{1}, \tau\right)}$ (a soma é normalizada pela raiz quadrada do volume do bloco, $L^{d / 2}$ ). Já a operação $\left(B^{*} v\right)_{\left(\theta_{1}, \tau\right)}$ consiste em atribuir a quantidade $\frac{1}{L^{d / 2}} v_{\tau}$ a todos os pontos do primeiro bloco hierárquico indexado por $\tau$. 
Definimos em $\mathbb{R}^{n}$ a matriz real simétrica

$$
J=\sum_{k=1}^{K} L^{-2 k}\left(B^{*}\right)^{k} B^{k}
$$

cuja forma quadrática associada, para $L^{d}=2$ e $d=2 /(\gamma-1)$, coincide com a energia hierárquica (3.1.12); reescrevemos esta como

$$
H=-\sum_{k=1}^{K} 2^{-\gamma k} \sum_{r=1}^{2^{K-k}}\left(\sigma_{k, r}\right)^{2}
$$

sendo

$$
\sigma_{k, r}=\sum_{(r-1) 2^{k}<j \leq r 2^{k}} \sigma_{j}
$$

a variável de bloco de $k$-ésimo nível hierárquico. Como visto, $\gamma(d)=1+2 / d$ varia de 2 a 1 à medida que $d$ varia de 2 a infinito. Se fizermos $L^{d}=2$, temos de (5.2.35) que $\sigma_{k, r}=2^{k / 2}\left(B^{k} \sigma\right)_{\theta}$ para algum $\theta \in \Lambda_{K-k}$, e que

$$
\begin{aligned}
-H & =-\sum_{k=1}^{K} 2^{-\gamma k+k} \sum_{\theta}\left(B^{k} \sigma\right)_{\theta}^{2}=-\sum_{k=1}^{K} 2^{-2 k / d}\left(B^{k} \sigma, B^{k} \sigma\right)_{\Lambda_{K-k}} \\
& =-\sum_{k=1}^{K} L^{-2 k}\left(\sigma,\left(B^{*}\right)^{k} B^{k} \sigma\right)_{\Lambda_{K}}=(\sigma, J \sigma)_{\Lambda_{K}},
\end{aligned}
$$

como afirmado anteriormente.

O laplaciano hierárquico $-\Delta_{H}$, sendo um gerador estocástico de um semi-grupo, satisfaz a

$$
-\Delta_{H} 1=0
$$

com $1=(1, \ldots, 1) / \sqrt{n}$ o vetor constante de norma $(1,1)_{\Lambda_{K}}=1 \mathrm{em} \mathbb{R}^{\Lambda_{K}}$. Dessa forma, podemos decompor o operador $J$ como a soma 


$$
J=\Delta_{H}+\mu_{0} I
$$

com $\mu_{0}$ obtida a partir de (5.2.35), (5.2.36) e (5.2.37):

$$
\begin{aligned}
\mu_{0} & =(1, J 1)_{\Lambda_{K}}=\sum_{k=1}^{K} L^{-2 k}\left(1,\left(B^{*}\right)^{k} B^{k} 1\right)_{\Lambda_{K}} \\
& =\sum_{k=1}^{K} L^{-2 k}\left(B^{k} 1, B^{k} 1\right)_{\Lambda_{K-k}} \\
& =\sum_{k=1}^{K} L^{-2 k}=\frac{1-L^{-2 K}}{L^{2}-1},
\end{aligned}
$$

uma vez que $\left(B^{k} 1\right)_{\theta}=L^{d(k-K) / 2}$ para todo $\theta \in \Lambda_{K-k}$, e conseqüentemente

$$
\left(B^{k} 1, B^{k} 1\right)_{\Lambda_{K-k}}=\sum_{k=1}^{L^{d(K-k)}} L^{d(k-K)}=1
$$

Temos assim que

$$
-\Delta_{H}=\sum_{k=1}^{K} L^{-2 k}\left(-\left(B^{*}\right)^{k} B^{k}+I\right) .
$$

Nossa meta é a construção de uma teoria espectral para o laplaciano hierárquico. É simples verificar através das definições de $B$ e $B^{*}$ que

$$
B^{k}\left(B^{*}\right)^{k}=B B^{*}=I
$$

segue para todo $k=1, \ldots, K$. Definimos

$$
P_{k}=\left(B^{*}\right)^{k} B^{k}
$$


um projetor (vale a identidade $P_{k}^{2}=P_{k}$ ) no subespaço dos vetores em $\mathbb{R}^{\Lambda_{K}}$ que assumem valores constantes sobre blocos de tamanho $L^{d k}$. De fato, a aplicação de $P_{k}$ sobre um vetor $u=\left(u_{\tau}\right)$ é tal que $P_{k}$ "homogeiniza" o $k$-ésimo bloco hierárquico que contém $\tau$. Isso porque as $k$ aplicações sucessivas de $B$ somam todas as componentes $u_{\left(\theta_{1}, \ldots, \theta_{k}, \tau\right)}$ contidas no $k$-ésimo bloco indexado por $\tau$, enquanto que as $k$ aplicações de $B^{*}$ distribuem uniformemente o resultado da soma por todos os pontos deste bloco.

Provemos que (5.2.41) é de fato um projetor. Seguem de (5.2.40) as relações

$$
\begin{aligned}
P_{k}^{2} & =\left(B^{*}\right)^{k} B^{k}\left(B^{*}\right)^{k} B^{k}=\left(B^{*}\right)^{k} I B^{k}=P_{k} ; \\
P_{j} P_{k} & =\left(B^{*}\right)^{j} B^{j}\left(B^{*}\right)^{k} B^{k}=\left(B^{*}\right)^{j} B^{j-k} B^{k}=P_{j} \\
P_{k} P_{j} & =\left(B^{*}\right)^{k} B^{k}\left(B^{*}\right)^{j} B^{j}=\left(B^{*}\right)^{k}\left(B^{*}\right)^{j-k} B^{j}=P_{j},
\end{aligned}
$$

válidas para todo $j>k$; temos portanto a seguinte inclusão

$$
P_{K}<P_{K-1}<\cdots<P_{1}<P_{0} \equiv I
$$

no sentido que $A<B$ se, e somente se, $(u, A v)_{\Lambda_{K}}<(u, B v)_{\Lambda_{K}}$ segue para todo $u \in \mathbb{R}^{\Lambda_{K}}$.

Sejam

$$
Q_{k}=P_{k}-P_{k+1}
$$

para $k=0,1, \ldots, K-1 \mathrm{e}$

$$
Q_{K}=P_{K}
$$

os operadores de flutuação de bloco. Podemos finalmente enunciar

Teorema 5.2.1 (Espectral [18]) A coleção $\left\{Q_{k}\right\}_{k=0}^{K}$ de matrizes projetoras ortogonais reais $n \times n$ 


$$
Q_{j} Q_{k}=\delta_{j k} Q_{k}
$$

constitui a decomposição espectral da identidade

$$
I=\sum_{k=0}^{K} Q_{k}
$$

$e$

$$
f\left(-\Delta_{H}\right)=\sum_{k=0}^{K} f\left(\lambda_{k}\right) Q_{k}
$$

segue com

$$
\lambda_{k}=\frac{L^{-2 k}-L^{-2 K}}{L^{2}-1}
$$

para qualquer função contínua $f:\left[0,1 /\left(L^{2}-1\right)\right] \longrightarrow \mathbb{R}$. Concluímos portanto que $-\Delta_{H}$ é uma matriz positiva, com $\lambda_{k}, k=0, \ldots, K-1$, um autovalor de multiplicidade $L^{d(K-k)}\left(1-L^{-d}\right)$ e $\lambda_{K}=0$ um autovalor simples.

Prova. Comecemos demonstrando a ortogonalidade dos projetores $Q_{k}$. De $(5.2 .42)$ e $(5.2 .44)$

$$
\begin{aligned}
Q_{j} Q_{k} & =\left(P_{j}-P_{j+1}\right)\left(P_{k}-P_{k+1}\right) \\
& =\left(P_{j}-P_{j}\right)-\left(P_{j+1}-P_{j+1}\right)=0
\end{aligned}
$$

para todo $k<j<K$; o mesmo segue para $j<k<K$. Para $j<k=K$,

$$
Q_{j} Q_{K}=\left(P_{j}-P_{j+1}\right) P_{K}=P_{K}-P_{K}=0
$$

e para $j=k$, 
$Q_{k} Q_{k}=\left(P_{k}-P_{k+1}\right)\left(P_{k}-P_{k+1}\right)=\left(P_{k}-P_{k+1}\right)-\left(P_{k+1}-P_{k+1}\right)=Q_{k}$

Com isso concluímos a prova de (5.2.45). Por definição,

$$
\sum_{k=0}^{K} Q_{k}=\sum_{k=0}^{K-1}\left(P_{k}-P_{k+1}\right)+P_{K}=P_{0}-P_{K}+P_{K}=I .
$$

Resta-nos provar (5.2.46) e (5.2.47). Não obstante, de (5.2.39), (5.2.41) e (5.2.44), temos que

$$
\begin{aligned}
-\Delta_{H} & =\sum_{j=1}^{K} L^{-2 j}\left(-P_{j}+I\right)=\sum_{j=1}^{K} L^{-2 j} \sum_{k=0}^{j-1} Q_{k} \\
& =\sum_{k=0}^{K-1}\left(\sum_{j=k+1}^{K} L^{-2 j}\right) Q_{k} \\
& =\sum_{k=0}^{K-1}\left(\frac{L^{-2 k}-L^{-2 K}}{L^{2}-1}\right) Q_{k}+0 \cdot Q_{K} \\
& =\sum_{k=0}^{K} \lambda_{k} Q_{k},
\end{aligned}
$$

precisamente (5.2.46) com $f(x)=x$. Segue de (5.2.44) que (5.2.46) é válida para qualquer polinômio, e pelo teorema de aproximação de Weierstrass, para qualquer função uniformemente contínua de $-\Delta_{H}$.

Já que $P_{k}$ projeta qualquer vetor de $\Lambda_{K}$ em vetores constantes sobre blocos disjuntos de tamanho $L^{d k}$, a multiplicidade de $P_{k}$ é $L^{d(K-k)}$, uma vez que há exatamente esta quantidade de blocos de $k$-ésimo nível hierárquico em um bloco de $K$-ésimo nível (trata-se da razão entre os volumes dos blocos).

Pela definição (5.2.44), juntamente com as inclusões (5.2.43), concluímos que a multiplicidade do operador de flutuação de bloco é $L^{d(K-k)}-L^{d(K-k-1)}$ para $k=1, \ldots, K-1$ e 1 para $K=1$, o que encerra a prova do teorema. 


\subsubsection{Ordem de longo alcance na ausência de campo}

Tendo em mãos o Teorema espectral 5.2.1, podemos aplicar o método do limite infravermelho para o modelo N-vetorial hierárquico. Comecemos pelo problema sem campo.

A medida de equilíbrio do modelo $\mathrm{N}$-vetorial hierárquico no hipercubo $\Lambda_{K}$ se exprime como

$$
d \nu_{\Lambda_{K}}(\sigma)=\frac{1}{Z_{\Lambda_{K}}(\beta)} \exp \left\{-\frac{\beta}{2}(\sigma(\theta), A \sigma(\theta))\right\} \prod_{i=1}^{n} d \sigma\left(\theta_{i}\right) \delta\left(\left|\sigma\left(\theta_{i}\right)\right|^{2}-1\right)
$$

em que $\sigma(\theta)=\left(\sigma\left(\theta_{1}\right), \ldots, \sigma\left(\theta_{n}\right)\right)$ é $n$-tupla de vetores em $\mathbb{R}^{N}, A=J \otimes I$ é o produto tensorial da matriz de acoplamento $J$ com a matriz identidade $I_{N \times N} ; Z_{\Lambda_{K}}(\beta)$ representa a função de partição

$$
Z_{\Lambda_{K}}(\beta)=\int_{\mathbb{R}^{n}} \exp \left\{-\frac{\beta}{2}(\sigma(\theta), A \sigma(\theta)) \prod_{i=1}^{n} d \sigma\left(\theta_{i}\right) \delta\left(\left|\sigma\left(\theta_{i}\right)\right|^{2}-1\right)\right\}
$$

o produto interno em $\mathbb{R}^{n N}$ é dado por $(x, y)=\sum_{i=1}^{n} x_{i} \cdot y_{i}$.

Na Seção 5.1, identificamos o quadrado da magnetização espontânea $m^{2}(0)$ com o modo zero da transformada de Fourier da função de spin, de energia zero (nesta representação o hamiltoniano era diagonal), e então usamos a regra de soma para demonstrar a ordem de longo alcance. Visamos estender esta idéia; mais especificamente, identificamos $m^{2}(0)$ com a média térmica do quadrado do modo de energia zero do hamiltoniano hierárquico (representado pela autofunção do projetor $Q_{K}$ de acordo com o Teorema 5.2.1).

Podemos, em vista do mesmo teorema, escrever qualquer vetor $u \in \mathbb{R}^{\Lambda_{K}} \mathrm{em}$ termos de sua decomposição espectral

$$
u_{\theta}=\sum_{j=0}^{K}\left(Q_{j} u\right)_{\theta}
$$

para todo $\theta \in \Lambda_{K}$. Por fim, a regra de soma (5.1.4) pode ser reescrita como 


$$
\frac{1}{\left|\Lambda_{K}\right|} \sum_{i=1}^{N}\left\langle\left(\sigma_{i}, \sigma_{i}\right)\right\rangle_{\nu}=\frac{1}{L^{d K}}\left[\sum_{j=0}^{K-1} \sum_{l=1}^{L^{d(K-j-1)}} \sum_{m=1}^{L^{d}-1}\left\langle\sigma^{2}(j, l, m)\right\rangle_{\nu}+\left\langle\sigma^{2}(K)\right\rangle_{\nu}\right]=1,
$$

com

$$
\sigma(j, l, m)=(y(j, l, m), \sigma)
$$

a projeção de $\sigma=\left(\sigma_{1}, \ldots, \sigma_{N}\right)$ em $y(j, l, m)$, autovetor normalizado de $Q_{j}$. Os índices $m$ e $l$ representam, respectivamente, os autovetores de $Q_{j}$ associados a um bloco de nível hierárquico $j+1$ (o espaço vetorial formado por estes vetores é de dimensão $L^{d}-1, L^{d}$ o número de blocos de nível $j$ em um bloco de nível $j+1$ ) e os blocos $j+1$ pertencentes ao bloco $\Lambda_{K}$ (há $L^{d(K-j-1)}$ destes). Há no total $L^{d(K-j)}-L^{d(K-j-1)}$ autovetores associados a um único $\lambda_{j}$, valor este que corresponde exatamente ao posto de $Q_{j}$. A esperança é tomada com respeito ao estado de Gibbs (5.2.48).

Como previamente discutido, a identificação do quadrado da magnetização espontânea com o modo zero é imediata:

$$
m^{2}(0)=\lim _{K \rightarrow \infty}\left[\left\langle\left(\frac{1}{L^{d K}} \sum_{\theta} \sigma(\theta)\right)^{2}\right\rangle_{\nu}\right]=\lim _{K \rightarrow \infty}\left[\frac{1}{L^{d K}}\left\langle\sigma^{2}(K)\right\rangle_{\nu}\right],
$$

já que $\sigma^{2}(K)$ corresponde justamente ao quadrado da soma das variáveis de spin no bloco $K$. Haverá magnetização espontânea caso

$$
m^{2}(0)=1-\lim _{K \rightarrow \infty}\left[\frac{1}{L^{d K}} \sum_{j=0}^{K-1} \sum_{l=1}^{L^{d(K-j-1)}} \sum_{m=1}^{L^{d}-1}\left\langle\sigma^{2}(j, l, m)\right\rangle_{\nu}\right]>0
$$

isto é, deve valer o limite

$$
\lim _{K \rightarrow \infty}\left[\frac{1}{L^{d K}} \sum_{j=0}^{K-1} \sum_{l=1}^{L^{d(K-j-1)}} \sum_{m=1}^{L^{d}-1}\left\langle\sigma^{2}(j, l, m)\right\rangle_{\nu}\right]<1
$$

$\operatorname{para} \beta>\beta_{c}$. 
Da mesma forma que tratamos o modelo N-vetorial com o laplaciano discreto, buscaremos obter o limite infravermelho a partir da dominação gaussiana (5.1.11) (cuja prova se encontra em detalhes no apêndice C), com $\eta$ e $Z_{\Lambda_{H}}(\eta)$ como em (5.1.12) e (5.1.13), respectivamente (obviamente a função de partição apropriada é a associada à medida (5.2.48)). A dominação gaussiana, juntamente com a utilização de condição de fronteira livre (a medida $\nu$ é invariante por mudança de sinal)

$$
\left\langle\left(\eta, \Delta_{H} \sigma\right)\right\rangle_{\nu}=\sum_{\theta \in \Lambda_{K}}(-\Delta \eta)(\theta)\langle\sigma(\theta)\rangle_{\nu}=0
$$

nos conduz a mesma equação (5.1.17)

$$
\left\langle\left(\eta,-\Delta_{H} \sigma\right)\left(\eta,-\Delta_{H} \sigma\right)\right\rangle_{\nu} \leq \frac{1}{\beta}\left(\eta,-\Delta_{H} \eta\right)
$$

Se tomarmos $\eta=\left(\eta_{1}, \ldots, \eta_{N}\right)$ como

$$
\eta_{j}=y_{j}(j, l, m) \delta_{i, j} \quad j=1, \ldots, N
$$

chegamos a

$$
\left\langle\sigma^{2}(j, l, m)\right\rangle_{\nu} \leq \frac{N}{\beta \lambda_{j}} \quad j \neq K
$$

expressão equivalente a (5.1.19). Derivemos explicitamente (5.2.52). De fato, como

$$
\begin{aligned}
\left(\eta,-\Delta_{H} \sigma\right) & =\left(-\Delta_{H} y(j, l, m), \sigma\right) \\
& =\left(\lambda_{j} y(j, l, m), \sigma\right) \\
& =\lambda_{j} \sigma_{i}(j, l, m)
\end{aligned}
$$

segue imediatamente

$$
\left\langle\left(\eta,-\Delta_{H} \sigma\right)\left(\eta,-\Delta_{H} \sigma\right)\right\rangle_{\nu}=\lambda_{j}^{2}\left\langle\sigma_{i}^{2}(j, l, m)\right\rangle_{\nu}
$$


Temos também que

$$
\begin{aligned}
\left(\eta,-\Delta_{H} \eta\right) & =\left(y(j, l, m),-\Delta_{H} y(j, l, m)\right) \\
& =\lambda_{j}\left(y_{i}(j, l, m), y_{i}(j, l, m)\right) \\
& =\lambda_{j} .
\end{aligned}
$$

A substituição destes resultados em (5.2.51) nos conduz a (5.2.52). Combinando (5.2.47) e (5.2.52) obtemos

$$
\begin{aligned}
m^{2}(0) & \geq 1-\lim _{K \rightarrow \infty}\left[\frac{1}{L^{d K}} \sum_{j=0}^{K-1} \sum_{l=1}^{L^{d(K-j-1)}} \sum_{m=1}^{L^{d}-1} \frac{N}{\beta \lambda_{j}}\right] \\
& =1-\frac{N}{\beta} \lim _{K \rightarrow \infty}\left[\frac{1}{L^{d K}} \sum_{j=0}^{K-1}\left(L^{d}-1\right)\left(L^{2}-1\right) \frac{L^{d(K-j-1)}}{L^{-2 j}-L^{-2 K}}\right] \\
& =1-\frac{N}{\beta}\left(1-L^{-d}\right)\left(L^{2}-1\right) \lim _{K \rightarrow \infty}\left[\sum_{j=0}^{K-1} \frac{L^{-d j}}{L^{-2 j}-L^{-2 K}}\right] \\
& =1-\frac{N}{\beta}\left(1-L^{-d}\right)\left(L^{2}-1\right) \sum_{j=0}^{\infty} L^{-(d-2) j} .
\end{aligned}
$$

A soma em (5.2.53) convergirá caso $d-2>0$, isto é, caso $d \geq 3$. Sob esta condição,

$$
m^{2}(0) \geq 1-\frac{N}{\beta}\left(L^{2}-1\right) \frac{1-L^{-d}}{1-L^{-(d-2)}} .
$$

Logo, se $\beta>\bar{\beta} \equiv N\left(L^{2}-1\right) \frac{1-L^{-d}}{1-L^{-(d-2)}}$ e $d \geq 3$ segue (5.2.50), e portanto o modelo N-vetorial hierárquico apresenta ordem de longo alcance a temperatura suficientemente baixa.

Por fim, provamos na próxima subseção a existência de ordem de longo alcance para o modelo N-vetorial hierárquico na presença de campo externo.

\subsubsection{Na presença de campo quase aleatório}

Consideremos o mesmo modelo N-vetorial hierárquico estudado até então com o hamiltoniano 


$$
-H_{\Lambda_{K}}(\sigma, h)=(\sigma, A \sigma)-(\sigma, h)
$$

$h$ o campo externo quase aleatório.

Definimos a nova média térmica através de (5.1.23), com $H$ dado por (5.2.54). O argumento central novamente se baseia na dominação gaussiana para o novo hamiltoniano, expressa por (5.1.24), com $Z_{\Lambda_{K}}(\eta, h)$ definido em (5.1.25). A prova é semelhante a apresentada na Subseção 5.1.2, e portanto detalhes são omitidos.

Precisamos, no entanto, mostrar que o funcional $\langle\cdot\rangle_{\nu}$ ( $\nu$ é a medida de Gibbs (5.2.48) com $H$ definido por (5.2.54)) é positivo por reflexão. Na realidade, podemos reescrever a interação dos spins com o campo como

$$
\begin{aligned}
(\sigma, h) & =\sum_{x \in \Lambda} \sigma(x) h(x)=\sum_{x \in \Lambda_{+}} \sigma(x) h(x)+\sum_{\pi_{P} x \in \Lambda_{-}} \sigma\left(\pi_{P} x\right) h\left(\pi_{P} x\right) \\
& =\sum_{x \in \Lambda_{+}} \sigma(x) h(x)+\pi_{P}\left(\sum_{x \in \Lambda_{+}} \sigma(x) h(x)\right)
\end{aligned}
$$

$\pi_{P}$ o morfismo com respeito ao plano $P$ que homogeiniza o campo externo, isto é, que associa a cada elemento de $\Lambda_{+}$um elemento de $\Lambda_{-}$(o morfismo atribui a $h\left(\pi_{P} x\right)$ o mesmo sinal de $h(x)$ ). Provemos que a interação hierárquica entre os spins de bloco é invariante por $\pi_{P}$. Suponha que a reflexão se realiza entre $k$-blocos. De acordo com (C.4), caso $l<k,(y, y)_{\Lambda_{K-l}} \geq 0$ independentemente do sinal de $\pi_{P}$. Caso $l \geq k$ e $\pi_{P}$ seja negativo, temos de (C.5)

$$
\begin{aligned}
(y, y)_{\Lambda_{K-l}} & =\|y\|_{+}^{2}+\left(\pi_{P}\|y\|_{+}\right)^{2}+2 \sum_{\tau} y_{\tau}^{+} \pi_{P} y_{\tau}^{+} \\
& \geq\|y\|_{+}^{2}+\left(\pi_{P}\|y\|_{+}\right)^{2}-2\|y\|_{+} \pi_{P}\|y\|_{+}=\left(\|y\|\left\|_{+}-\pi_{P}\right\| y \|_{+}\right)^{2} \geq 0,
\end{aligned}
$$

pela desigualdade de Cauchy-Schwarz, o que mostra a positividade por reflexão da interação $A$ (vide Apêndice C).

Da dominação gaussiana (cuja prova se encontra no apêndice B) extraímos a desigualdade 


$$
\left\langle\exp \left\{\beta\left(\eta, \Delta_{H} \sigma\right)\right\}\right\rangle_{\nu} \leq \exp \left\{\beta\left(\eta,-\frac{\Delta_{H}}{2} \eta\right)+\beta(\eta, h)\right\}
$$

diferindo de (5.1.27) apenas no laplaciano.

Iniciemos nosso estudo pelo campo alternado. Em analogia aos resultados da Subseção 5.1.2, sabemos que os autovetores de $-\Delta_{H}$ são ortogonais ao campo, com exceção de $L^{d(K-1)}$ autovetores do projetor $Q_{0}$, associados às flutuações dos spins no primeiro nível hierárquico (há no sistema $L^{d(K-1)}$ blocos desse nível, com cada bloco apresentando $L^{d}-1$ autovetores de $Q_{0}$; somente um destes não é ortogonal ao campo alternado).

Logo, se levarmos em conta que $\left\langle\left(\eta, \Delta_{H} \sigma\right)\right\rangle_{\nu}=0$ para uma condição de fronteira livre e para todo $\eta \neq y(0, l, 1)$ (representamos o modo alternado pelo índice $m=1$ na terceira entrada), temos da expansão de (5.2.55) até segunda ordem em $\eta$ que

$$
\left\langle\left(\eta,-\Delta_{H} \sigma\right)\left(\eta,-\Delta_{H} \sigma\right)\right\rangle_{\nu} \leq \frac{1}{\beta}\left(\eta,-\Delta_{H} \eta\right)
$$

Portanto, para todos $j \neq 0, l \in\left\{1, \ldots, L^{d(K-j-1)}\right\}$ e $m \in\left\{1, \ldots, L^{d}-1\right\}$ tais que $(y(j, l, m), h)=0$, asseguramos a validade do limite infravermelho (5.2.52).

Obtemos um limitante superior de $\left\langle\sigma^{2}(0, l, 1)\right\rangle_{\nu}$ através do mesmo procedimento descrito na Subseção 5.1.2: extraímos da dominação gaussiana (5.2.55) um limitante de $\langle\sigma(0, l, 1)\rangle_{\nu}$, e em seguida assumimos que, no limite termodinâmico, $\left\langle\sigma^{2}(0, l, 1)\right\rangle_{\nu}=\langle\sigma(0, l, 1)\rangle_{\nu}^{2}$ (ou seja, a variância de $\sigma(0, l, 1)$ é de ordem inferior ao volume do sistema).

Se substituírmos $\eta=y(0, l, 1)$ por $\lambda \eta$ em (5.2.55) e efetuarmos sua expansão em série de Taylor temos

$$
\lim _{\lambda \rightarrow 0}\left[\left\langle\exp \left\{\beta\left(\lambda \eta, \Delta_{H} \sigma\right)\right\}\right\rangle_{\nu}-1\right] \lambda^{-1}=\lim _{\lambda \rightarrow 0}\left\{\beta\left\langle\left(\eta, \Delta_{H} \sigma\right)\right\rangle_{\nu}+O(\lambda)\right\}
$$

para o membro esquerdo de $(5.2 .55)$ e

$$
\lim _{\lambda \rightarrow 0}\left[\exp \left\{\beta\left(\lambda \eta,-\frac{\Delta_{H}}{2}(\lambda \eta)+h\right)\right\}-1\right] \lambda^{-1}=\lim _{\lambda \rightarrow 0}\{\beta(\eta, h)+O(\lambda)\}
$$


para o membro direito. Obtemos das igualdades acima e de (5.2.55)

$$
\left|\langle\sigma(0, l, 1)\rangle_{\nu}\right| \leq \frac{N \epsilon L^{d / 2}}{\lambda(0)}
$$

para cada $l \in\left\{1, \ldots, L^{d(K-1)}\right\}$.

Podemos, portanto, escrever o quadrado da magnetização como função de $h$ através da expressão

$$
\begin{aligned}
m^{2}(h) & =1-\lim _{K \rightarrow \infty}\left[\frac{1}{L^{d K}} \sum_{j=1}^{K-1} \sum_{l=1}^{L^{d(K-j-1)}} \sum_{m=1}^{L^{d}-1}\left\langle\sigma^{2}(j, l, m)\right\rangle_{\nu}-\frac{1}{L^{d K}} \sum_{l=1}^{L^{d(K-1)}} \frac{N^{2} \epsilon^{2} L^{d}}{\lambda^{2}(0)}\right] \\
& \geq 1-\frac{N}{\beta}\left(1-L^{-d}\right)\left(L^{2}-1\right) \sum_{j=1}^{\infty} L^{-(d-2) j}-N^{2} \epsilon^{2}\left(L^{2}-1\right)^{2}
\end{aligned}
$$

com $\lim _{K \rightarrow \infty} \lambda(0)=\left(L^{2}-1\right)^{-1}$, de acordo com (5.2.47). A soma em (5.2.57) somente converge se $d \geq 3$; sob esta condição,

$$
m^{2}(h) \geq 1-\frac{N}{\beta}\left(L^{2}-1\right) \frac{1-L^{-d}}{L^{d-2}-1}-N^{2} \epsilon^{2}\left(L^{2}-1\right)^{2},
$$

estritamente positivo caso $\beta \geq \bar{\beta} \equiv \frac{N\left(L^{2}-1\right)\left(1-L^{-d}\right)}{\left(L^{d-2}-1\right)\left(1-N^{2} \epsilon^{2}\left(L^{2}-1\right)^{2}\right)}$, com $\epsilon<\left[N\left(L^{2}-1\right)\right]^{-1}$. Concluímos assim que o modelo N-vetorial hierárquico com campo externo alternado, de intensidade fraca, apresenta ordem de longo alcance à temperatura suficientemente baixa. Há, como para o modelo com laplaciano usual, uma competição entre a interação dos spins com o campo e o ferromagnetismo.

Passemos para o estudo do campo quase aleatório. Podemos reescrevê-lo como

$$
h=h_{\mathrm{alt}}+\zeta,
$$

com $h_{\text {alt }}$ o campo alternado e $\zeta$ definido por

$$
\zeta=\chi\left(\Lambda_{\mathrm{ir}}\right)\left(-h_{\mathrm{alt}} \pm \epsilon\right)
$$


$\chi\left(\Lambda_{\text {ir }}\right)$ a função indicadora dos blocos irregulares (note que, por construção, $\zeta$ se anula fora desses blocos). Visamos com a definição (5.2.58) tratar as regiões irregulares como uma perturbação ao campo alternado.

Façamos mais uma vez tais blocos serem esparsos. Para tanto, utilizemos a escala definida pela seqüência $f_{n}\left(L_{n}\right)=M^{n} L_{n}, M \in \mathbb{N}, M>1$ e $L_{1}=L$ (a letra $K$ designa neste contexto o mais alto nível hierárquico do sistema, e assim a substituímos por $M)$.

Tendo em mãos o resultado para o campo alternado, necessitamos de uma estimativa para o campo $\zeta$. Para tanto, devemos verificar quais autovetores de $-\Delta_{H}$ não são ortogonais ao campo (para os demais modos podemos empregar o limite infravermelho (5.2.52), uma vez que suas médias térmicas se anulam graças à simetria da medida de Gibbs; vide Subseção 5.1.2 para maiores esclarecimentos).

Suponhamos que estejamos interessados em um bloco $j$-irregular. Vemos da definição (5.2.59) que o campo nesta região é ortogonal aos autovetores dos projetores $Q_{k}$, com exceção dos projetores $Q_{0}$ e $Q_{j}$ : há para $Q_{0}$ um autovetor proporcional ao campo; assumiremos que nenhum autovetor de $Q_{j}$ é ortogonal ao campo (há para cada $j+1$-bloco $M^{j d}-1$ autovetores de $Q_{j}$ ).

Novamente obtemos um limitante superior para os modos que não são ortogonais ao campo (que apresentam ocupação macroscópica, como vimos nos casos anteriores) a partir do procedimento adotado até então: extraímos da dominação gaussiana (5.2.55) um limitante para a média térmica destes modos, e em seguida fazemos a seguinte

Hipótese 5.2.2 As variâncias das variáveis de spin projetadas em qualquer um dos modos não ortogonais ao campo quase aleatório são de ordem o $(|\Lambda|)$.

Ao substituírmos $\eta=y(j, l, m)$ por $\lambda \eta$ em (5.2.55), obtemos, expandindo a relação até termos de primeira ordem,

$$
\left|\langle\sigma(j, l, m)\rangle_{\nu}\right| \leq \frac{1}{\lambda(j)}(\zeta, y(j, l, m)) \leq \frac{2 N \epsilon L_{j}^{d}}{\lambda(j) L_{j+1}^{d / 2}},
$$

já que a contribuição é, no máximo, a do volume dos dois blocos $j$-irregulares; o autovetor $y(j, l, m)$ é normalizado pela raiz quadrada do volume do bloco 
$j+1$-admissível. A desigualdade (5.2.60) é válida para todo $l \in\{1, \ldots, \alpha(j+1)\}$ e todo $m \in\left\{1, \ldots, M^{j d}-1\right\}$, com $\alpha(j+1)$ o número de blocos $j+1$-admissíveis presentes em um bloco $K$-admissível:

$$
\alpha(j+1)=\left(M^{d(K-1)}-2\right) \cdots\left(M^{d(j+1)}-2\right)=\prod_{i=j+1}^{K-1}\left(M^{d i}-2\right) .
$$

Por outro lado, a estimativa de $\langle\sigma(0, l, m)\rangle_{\nu}$ é mais uma vez dada por (5.2.56), com $m \in\left\{1, \ldots, L_{j}^{d} / L^{d}\right\}$ (há $L_{j}^{d} / L^{d} 1$-blocos em um $j$-bloco). Desse modo, podemos exprimir o quadrado da magnetização como função de $h$ a partir de

$$
\begin{aligned}
m^{2}(h) \geq 1-\lim _{K \rightarrow \infty}\left[\frac{1}{L_{K}^{d}} \sum_{j=1}^{K-1} \sum_{l=1}^{L_{K}^{d} / L_{j+1}^{d}} \sum_{m=1}^{L_{j+1}^{d} / L_{j}^{d}-1} \frac{N}{\beta \lambda(j)}\right] \\
-\lim _{K \rightarrow \infty}\left[\frac{1}{L_{K}^{d}} \sum_{l=1}^{L_{K}^{d} / L_{j+1}^{d}} \sum_{m=1}^{L_{j+1}^{d} / L^{d}} \frac{N^{2} \epsilon^{2} L^{d}}{\lambda^{2}(0)}\right] \\
-\lim _{K \rightarrow \infty}\left[\frac{1}{L_{K}^{d}} \sum_{j=1}^{K-1} \sum_{l=1}^{\alpha(j+1)} \sum_{m=1}^{M^{d j}-1} \frac{4 N^{2} \epsilon^{2} L_{j}^{2 d}}{\lambda^{2}(j) L_{j+1}^{d}}\right] \\
-\lim _{K \rightarrow \infty}\left[\frac{1}{L_{K}^{d}} \sum_{l=1}^{2 \alpha(j+1)} \sum_{m=1}^{L_{j}^{d} / L^{d}} \frac{N^{2} \epsilon^{2} L^{d}}{\lambda^{2}(0)}\right]
\end{aligned}
$$

$\operatorname{com} \lambda_{j}=L^{-2} \sum_{k=j+1}^{K} M^{-k(k-1)}$ a generalização dos autovalores (5.2.47) para a escala $L_{n}=M^{n-1} L_{n-1}$. Se levarmos em conta que $\lim _{K \rightarrow \infty} \lambda(0) \equiv \bar{\lambda}(0)=L^{2} \sum_{1 \leq k \leq \infty} M^{-k(k-1)}$, que $\lambda_{j}>L^{-2} M^{-j(j+1)}$ e que $\alpha(j+1)<L_{K}^{d} / L_{j+1}^{d}$, extraímos de $(5.2 .61)$

$$
\begin{aligned}
m^{2}(h) \geq & 1-\frac{N L^{-(d-2)}}{\beta M^{-d}} \sum_{j=1}^{\infty} M^{-(d-2) j(j+1) / 2}-\frac{N^{2} \epsilon^{2}}{\bar{\lambda}^{2}(0)} \\
& -(2 N L \epsilon)^{2} \sum_{j=1}^{\infty} \frac{M^{2 j(j+1)}}{M^{d j}}-2 \frac{N^{2} \epsilon^{2}}{\bar{\lambda}^{2}(0)} .
\end{aligned}
$$

Entretanto, a segunda soma de (5.2.62) diverge para todo $d>0$, o que torna o método inaplicável para esta configuração de campo. Busquemos então uma 
escala que apresente crescimento super-exponencial, isto é, que seja definida por $f_{n}\left(L_{n}\right)=L_{n+1}=\left[L_{n}^{(\alpha-1)}\right] L_{n}, \alpha>1$, com $[x]$ a parte inteira de $x$ (visamos com isso preservar a estrutura dos blocos). A generalização dos resultados acima é imediata: o número de blocos $j+1$-admissíveis em um bloco $K$-admissível e os autovalores $\lambda(j)$ de $-\Delta_{H}$ passam a ser expressos, respectivamente, como

$$
\alpha(j+1)=\left(\left[L_{K-1}^{(\alpha-1)}\right]^{d}-2\right) \cdots\left(\left[L_{j+1}^{(\alpha-1)}\right]^{d}-2\right)
$$

e como

$$
\lambda(j)=\sum_{k=j+1}^{K} L_{k}^{-2}=\sum_{k=j+1}^{K} O\left(\left[L^{-2 \alpha^{k}}\right]\right) .
$$

Podemos neste momento enunciar a

Proposição 5.2.3 O modelo $N$-vetorial hierárquico com campo quase aleatório super-exponencialmente esparso apresenta, sob a Hipótese (5.2.2), ordem de longo alcance para todo $\beta>\bar{\beta}$, para $\epsilon<N^{-1}\left[\frac{3}{\bar{\lambda}^{2}(0)}+4 S_{2}\right]^{-1 / 2}$ e para $d>\max \left(2 \alpha, \frac{4 \alpha}{\alpha-1}\right)$, com

$$
\begin{gathered}
\bar{\beta} \equiv \frac{N S_{1} \bar{\lambda}(0)}{\bar{\lambda}(0)-N^{2} \epsilon^{2}\left[3+4 S_{2} \bar{\lambda}(0)\right]}, \\
S_{1} \equiv \sum_{j=1}^{\infty}\left[L_{j}^{(2 \alpha-d)}\right], \\
S_{2} \equiv \sum_{j=1}^{\infty}\left[L_{j}^{[4 \alpha-d(\alpha-1)]}\right]
\end{gathered}
$$

e

$$
\bar{\lambda}(0) \equiv \sum_{k=1}^{\infty}\left[L_{j}^{-2 \alpha}\right]
$$


A prova da proposição segue imediatamente da desigualdade (5.2.62), que para a escala de crescimento super-exponencial proposta se torna

$$
\begin{aligned}
m^{2}(h) \geq & 1-\frac{N}{\beta} \sum_{j=1}^{\infty}\left[L_{j}^{(2 \alpha-d)}\right]-\frac{N^{2} \epsilon^{2}}{\bar{\lambda}^{2}(0)} \\
& -4 N^{2} \epsilon^{2} \sum_{j=1}^{\infty}\left[L_{j}^{[4 \alpha-d(\alpha-1)]}\right]-2 \frac{N^{2} \epsilon^{2}}{\bar{\lambda}^{2}(0)} \\
= & 1-\frac{N S_{1}}{\beta}-4 S_{2} N^{2} \epsilon^{2}-3 \frac{N^{2} \epsilon^{2}}{\bar{\lambda}^{2}(0)},
\end{aligned}
$$

com $S_{1}, S_{2}$ e $\bar{\lambda}(0)$ definidos nesta ordem por (5.2.64), (5.2.65) e (5.2.66). Repare que $S_{1}$ e $S_{2}$ convergem somente se $d>2 \alpha$ e $d>\frac{4 \alpha}{\alpha-1}$, respectivamente (devemos tomar $d$ como o máximo entre esses dois números a fim de garantir a convergência das duas séries simultaneamente). Adotemos, por exemplo $\alpha=2$. Nesta situação, as desigualdades acima são satisfeitas para $d>8$.

Vemos de (5.2.62) que para $\beta>\bar{\beta}$ ( $\operatorname{com} \bar{\beta}$ definido por (5.2.63)) e para todo $\epsilon<N^{-1}\left[\frac{3}{\bar{\lambda}^{2}(0)}+4 S_{2}\right]^{-1 / 2}$, o quadrado da magnetização é estritamente positivo, o que indica existência de ordem de longo alcance. Isto encerra a prova. 


\section{Capítulo 6}

\section{Conclusões e problemas em aberto}

Nosso primeiro esforço foi verificar a persistência do argumento de Peierls (que estabelece um critério para ordem de longo alcance a baixa temperatura em sistemas com simetria discreta) para o modelo de Ising ferromagnético bidimensional com campo externo. A estabilidade do método depende do nosso sucesso em estimar a razão entre funções de partição internas a um dado contorno, oriunda da comparação entre as energias das configurações com e sem tal contorno. Caso a razão tenha peso energético de mesma ordem do contorno, o argumento é estável; caso seja de ordem superior, o argumento deixa de ser válido. No argumento original, o sistema não apresenta campo: a razão entre as funções de partição é identicamente um.

A primeira estrutura de campo estudada foi a alternada, regular em toda a rede. Por um argumento similar ao argumento de Peierls usual, eliminamos um contorno por uma tranformação que busca anular o efeito do campo, isto é, pela tranformação mapeamos a função de partição com condição de fronteira interna negativa na função com condição de fronteira interna positiva a menos de um termo de interação com a fronteira (o mapa desloca possíveis contornos internos que podem interagir com a fronteira), cujo peso energético apresenta a cardinalidade do contorno. Desse modo, conseguimos, com exceção deste termo de interação, eliminar a razão entre as funções de partição. O argumento segue para um campo de intensidade de desordem fraca $(\epsilon<1)$.

O resultado nos mostra que para $\epsilon$ pequeno, a alternância apresentada pelo 
campo não destrói o ordenamento natural dos spins decorrente da interação ferromagnética do modelo (o campo é neutro em todas as escalas). Obviamente esta configuração de campo magnético, que se tratando de uma variável aleatória, é improvável de ser gerada se tomarmos o campo independentemente para cada ponto da rede segundo uma distribuição de probabilidade não degenerada.

Em seguida, buscamos aplicar o argumento ao modelo na presença de um campo externo mais realístico do ponto de vista de um padrão aleatório, isto é, que além da alternância de sinal apresente em todas as escalas regiões alinhadas em ambas as direções (sendo, no entanto, globalmente neutro). Todavia, não obtivemos sucesso com o método, e o problema de estimar razões entre funções de partição para um modelo com campo quase aleatório permanece sem solução.

Buscamos analisar, como alternativa ao estudo da existência de ordem de longo alcance no modelo de Ising ferromagnético com campo, o modelo hierárquico de Dyson (MHD) com geometria bidimensional, uma extensão do resultado original de Dyson em uma dimensão. Verificamos a existência de uma fase ordenada em duas situações distintas: sem campo magnético e com campo magnético.

No caso da ausência de campo, mostramos a existência de ordem de longo alcance a baixas temperaturas através da generalização do importante Teorema 3.1.2. Para tanto, fizemos uso de uma relação de recorrência para o parâmetro de ordem $m^{2}$, construída a partir das variáveis de spin de bloco (uma variável de spin de bloco corresponde à soma normalizada dos spins que constituem o bloco), decorrente das desigualdades de Griffiths (para modelos ferromagnéticos) e de um lema probabilístico (Lema 3.1.4, adaptado de [5]). O resultado é análogo ao obtido para o modelo unidimensional.

Em seguida, verificamos se o modelo com campo quase aleatório, cuja configuração é apresentada na Seção 2.3 (tipicamente aleatória), preserva a ordem de longo alcance a baixa temperatura presente no modelo sem campo. Neste caso, uma relação de recorrência para o parâmetro de ordem $m^{2}$ depende de estimativas de esperanças que envolvem a eliminação do campo externo, que não sabemos tratar. Na realidade, necessitamos de uma relação de recorrência para os blocos admissíveis; a dificuldade está em tratar os blocos irregulares, que não apresentam 
nenhuma recorrência.

A solução que buscamos envolve a substituição dos termos com campo irregular por termos equivalentes sem campo, porém com temperatura maior (a eliminação do campo, que eventualmente é responsável por um ordenamento alternado dos spins, em oposição ao ordenamento uniforme, é compensada por um aumento de temperatura do sistema, que como sabemos também gera um aumento da desordem). Entretanto, vemos que dada a natureza da interação dos spins com o campo (a interação do campo é proporcional ao volume dos blocos irregulares que, por construção, crescem indefinidamente; já a interação ferromagnética do MHD se escreve em termos das variáveis de spin de bloco e não apresenta a mesma intensidade da interação do campo), a única forma de eliminá-lo é fazer a temperatura divergir.

Com o intuito de corrigirmos este problema sugerimos que os blocos irregulares presentes no campo sejam esparsos (o que significa que sua proporção no todo diminui com o aumento da escala), uma estrutura até mais realística que a anterior (a densidade das regiões irregulares cai com o aumento da região) para um campo aleatório . Dessa forma, garantimos que no limite termodinâmico a contribuição dos termos com campo irregular na relação de recorrência para $m^{2}$ desapareça, o que a torna uma relação que envolve apenas regiões alternadas. Utilizamos uma escala de crescimento exponencial $\left(L_{n}=K^{n-1} L_{n-1}, K\right.$ um número natural). Mostramos que, para todo $K>1$, o sistema apresenta ordem de longo alcance para baixas temperaturas e para campo suficientemente fraco. Vemos mais uma vez que a presença de um campo alternado (de intensidade pequena) não é suficiente para destruir o ordenamento apresentado pelo sistema a baixas temperaturas.

Estudamos também o modelo de contornos hierárquicos $(\mathrm{MCH})$, com o intuito de tornar mais completa nossa análise de existência de ordem de longo alcance em sistemas com simetria discreta. Do mesmo modo que tratamos o problema no MHD, buscamos uma relação de recorrência para a magnetização (na realidade, no MHD utilizamos como parâmetro de ordem o quadrado da magnetização dada a condição de fronteira empregada (livre); aqui, por conveniência e sem perda de generalidade, utilizamos a condição de fronteira como sendo positiva) . Contudo, o método empregado é ligeiramente diferente e envolve um procedimento de renormalização, 
escala por escala, do campo magnético.

Como usual, empregamos o campo externo quase aleatório em nossas análises. Verificamos que, no limite assintótico $\beta \rightarrow \infty$ (baixas temperaturas), a única situação na qual a magnetização é diferente de zero é a de um campo magnético nulo. Identificamos novamente os blocos irregulares presentes no campo como os responsáveis por este fenômeno. Aplicando o mesmo argumento de esparsidade utilizado no estudo do MHD (inclusive a mesma escala empregada), verificamos que, para todo $K>1$, o sistema magnetiza a baixas temperaturas. A conclusão que extraímos dos resultados é a mesma do MHD: o campo alternado preserva a fase ferromagnética do modelo sem campo.

Tendo em vista os resultados descritos acima, tanto para campo alternado como para campo quase aleatório com regiões irregulares esparsas, propomos a seguinte conjectura: o argumento de Peierls é estável (isto é, a ordem de longo alcance é preservada) caso as regiões de campo irregular sejam esparsas.

Por fim, estudamos como a ocorrência de magnetização espontânea na ausência de campo externo para o modelo N-vetorial (cujo modelo de Ising é um caso particular) se relaciona com o fenômeno de condensação do estado de momento nulo. Tendo-se compreendido o método, buscamos estendê-lo para o modelo na presença de campo externo. Obtivemos a dominação gaussiana para o campo alternado, oriundo da positividade por reflexão da medida de Gibbs do modelo. Todavia, não alcançamos o mesmo resultado para o campo quase aleatório, uma vez que a medida de Gibbs nesta situação não é positiva por reflexão (o método do limite infravermelho não é aplicável).

Em seguida, verificamos a validade do limite infravermelho para os modos do laplaciano ortogonais ao campo (suas médias térmicas se anulam, uma vez que a medida de Gibbs é invariante pelas trocas simultâneas $\sigma(x) \rightarrow-\sigma(x)$ e $h(x) \rightarrow-h(x)$ para todo $x \in \Lambda$ ). Entretanto, o limite deixa de valer para o modo em que a configuração dos spins é alternada (proporcional ao campo). A saber, ocorre uma condensação desse modo, o que indica a existência de uma competição entre o ferromagnetismo e a interação do sistema com o campo (alternado). Mostramos, para um campo de intensidade fraca, existência de ordem de longo alcance, sob a 
hipótese de que variância associada a esse modo do laplaciano apresente cardinalidade inferior a do volume do sistema. A prova dessa nossa hipótese representa um dos problemas em aberto. Vimos que no caso gaussiano, (as componentes do $\mathrm{N}$-vetor $\sigma$ são independentes entre si) a razão entre a variância e o volume do sistema vai a zero no limite termodinâmico, um indício de que nossa hipótese é correta.

Tratamos então o modelo N-vetorial hierárquico, decorrente da substituição do laplaciano discreto (de diferença finita) pelo laplaciano hierárquico. Visamos com isso tirar proveito da estrutura hierárquica do campo quase aleatório.

Antes de tratarmos o sistema com campo, estendemos o método do limite infravermelho para o modelo N-vetorial na ausência de campo. Novamente foi possível obter a dominação gaussiana, uma generalização do resultado obtido para o modelo com laplaciano discreto na ausência de campo. O resultado, análogo ao modelo usual, mostra existência de ordem de longo alcance (por meio da ocupação macroscópica do estado fundamental) para $d \geq 3$.

Somente então estudamos o modelo com campo, para o qual demonstramos novamente a dominação gaussiana. A obtenção desse resultado somente foi possível graças à positividade por reflexão da medida de Gibbs do modelo N-vetorial hierárquico, com campo quase aleatório, decorrente da natureza do laplaciano (a interação ferromagnética deste modelo não apresenta uma estrutura geométrica (todos os spins interagem entre si), diferentemente do que ocorre com o modelo usual, onde a interação se dá apenas entre os primeiros vizinhos). Em seguida, provamos mais uma vez a existência de ordem de longo alcance em $d \geq 3$ para o modelo com campo alternado, de intensidade fraca, novamente sob a hipótese de que a variância do modo alternado (autovetor do projetor $Q_{0}$ ) apresenta cardinalidade inferior a do volume do sistema.

Por fim, tratamos o campo quase aleatório como sendo um campo alternado perturbado por um campo com suporte nas regiões irregulares. Verificamos a validade do limite infravermelho para os modos do laplaciano ortogonais ao campo. Para os demais modos (em um bloco $j+1$-admissível, o campo perturbador é proporcional a uma parcela dos $L_{j+1}^{d} / L_{j}^{d}$ autovetores de $Q_{j}$ e aos $L_{j+1}^{d} / L^{d}$ autovetores de $Q_{0}$ ), fizemos uso da Hipótese 5.2.2. 
Em primeiro lugar, estudamos a situação em que os blocos irregulares são esparsos em uma escala que cresce exponencialmente, como feito nos modelos anteriores. O método, no entanto, não é aplicável nesta situação, já que não conseguimos controlar o efeito da condensação dos modos não ortogonais ao campo (há um número infinito deles). Propusemos então um argumento de esparsidade dos blocos irregulares em uma escala que apresenta crescimento super-exponencial (controlado por um parâmetro $\alpha$ ), visando atenuar esse efeito. Verificamos existência de ordem de longo alcance nestas circunstâncias, desde que o campo apresente intensidade suficientemente pequena e a rede apresente dimensão suficientemente grande (a saber, $d>\max \left(2 \alpha, \frac{4 \alpha}{\alpha-1}\right)$; vide Proposição 5.2.3).

Sintetizando, os sistemas bidimensionais com simetria discreta (modelo de Ising, MHD e MCH) apresentam, para temperatura baixa, ordem de longo alcance na presença de um campo alternado. Obtemos um resultado análogo ao anterior para os modelos N-vetoriais usual e hierárquico, de simetria contínua. Diferentemente dos modelos com simetria discreta, aos quais mostramos existência de ordem em $d=2$, os modelos N-vetoriais somente exibem, graças ao teorema de GoldstoneMermin-Wagner, transição para $d \geq 3$. Para o MHD e o MCH, a presença de blocos irregulares esparsos em meio ao campo alternado não destrói a fase ferromagnética presente no caso sem campo. A situação é mais adversa para o modelo N-vetorial, em que somente provamos ordem de longo alcance tornando os blocos irregulares erparsos em uma escala com crescimento super-exponencial. 


\section{Apêndice A}

\section{Prova do Lema 3.1.4}

Sejam $\varphi(a)$ e $\psi(a)$, respectivamente, os membros esquerdo e direito da desigualdade (3.1.24). Mostremos que $\psi(a)$ satisfaz a equação diferencial

$$
\left(1 \cdot \nabla_{a}\right) \psi+\psi^{2}-(1 \cdot \bar{\Lambda}) 1=0
$$

$\operatorname{com} \bar{\Lambda}:=\bar{R}^{2}$ e condição inicial $\psi(0)=0$. O operador

$$
\nabla_{a}:=\left(\frac{\partial}{\partial a_{1}}, \ldots, \frac{\partial}{\partial a_{d}}\right)
$$

é o operador gradiente com respeito à variável $a=\left(a_{1}, \ldots, a_{d}\right)$.

Reescrevamos $\psi(a)$ como

$$
\psi(a)=(1 \cdot \bar{R}) \tanh (\bar{R} a)=\sum_{\alpha, \beta} \bar{R}_{\alpha \beta} \tanh \left(\sum_{\gamma} \bar{R}_{\beta \gamma} a_{\gamma}\right)
$$

(note que $\psi(0)=0$ ). Da definição do operador $\nabla_{a}$ temos

$$
\left(1 \cdot \nabla_{a}\right) \psi=\sum_{\alpha, \beta, \epsilon} \bar{R}_{\alpha \beta} \frac{\partial}{\partial a_{\epsilon}} \tanh \left(\sum_{\gamma} \bar{R}_{\beta \gamma} a_{\gamma}\right)
$$

Como 


$$
\frac{\partial}{\partial a_{\epsilon}} \tanh \left(\sum_{\gamma} \bar{R}_{\beta \gamma} a_{\gamma}\right)=\bar{R}_{\beta \epsilon}\left(1-\tanh ^{2}\left(\sum_{\gamma} \bar{R}_{\beta \gamma} a_{\gamma}\right)\right)
$$

concluímos

$$
\begin{aligned}
\left(1 \cdot \nabla_{a}\right) \psi & =\sum_{\alpha, \epsilon}\left(\bar{R}^{2}\right)_{\alpha \epsilon}-\sum_{\alpha}(\bar{R} \tanh (\bar{R} a))_{\alpha} \sum_{\epsilon}(\bar{R} \tanh (\bar{R} a))_{\epsilon} \\
& =\sum_{\alpha}\left(\bar{R}^{2} 1\right)_{\alpha}-(1 \cdot \bar{R} \tanh (\bar{R} a))^{2}=(1 \cdot \bar{\Lambda}) 1-\psi^{2}
\end{aligned}
$$

em que fizemos uso da simetria da matriz $\bar{R}$. Assim,

$$
\left(1 \cdot \nabla_{a}\right) \psi+\psi^{2}-(1 \cdot \bar{\Lambda}) 1=(1 \cdot \bar{\Lambda}) 1-\psi^{2}+\psi^{2}-(1 \cdot \bar{\Lambda}) 1=0
$$

Portanto, $\psi(a)$ satisfaz (A.1) com a condição inicial $\psi(0)=0$. Diferenciando $\varphi$ com respeito a $a_{\alpha}$, somando sobre $\alpha$ e utilizando a simetria de $X$, obtemos

$$
\begin{aligned}
\left(1 \cdot \nabla_{a}\right) \varphi & =\sum_{\alpha} \frac{\partial}{\partial a_{\alpha}} \varphi \\
& =\frac{\mathbb{E}\left[(1 \cdot X) \sum_{\alpha} X_{\alpha} e^{a \cdot X}\right]}{\mathbb{E}\left[e^{a \cdot X}\right]}-\frac{\mathbb{E}\left[(1 \cdot X) e^{a \cdot X}\right] \mathbb{E}\left[\sum_{\alpha} X_{\alpha} e^{a \cdot X}\right]}{\left(\mathbb{E}\left[e^{a \cdot X}\right]\right)^{2}} \\
& =\frac{\mathbb{E}\left[(1 \cdot X)^{2} e^{a \cdot X}\right]}{\mathbb{E}\left[e^{a \cdot X}\right]}-\varphi^{2} .
\end{aligned}
$$

Definindo $\Lambda=\left[\Lambda_{\alpha \beta}\right]$ a partir da equação

$$
(1 \cdot \Lambda) 1=\frac{\mathbb{E}\left[(1 \cdot X)^{2} \cosh (a \cdot X)\right]}{\mathbb{E}[\cosh (a \cdot X)]}
$$

isto é, $\Lambda_{\alpha \beta}=\mathbb{E}\left[X_{\alpha} X_{\beta} \cosh (a \cdot X)\right] / \mathbb{E}[\cosh (a \cdot X)]$, podemos afirmar que $\varphi$ satisfaz a equação diferencial (A.1) (com $\bar{\Lambda}$ substituído por $\Lambda$ ) e a condição inicial $\varphi(0)=0$. Desse modo, caso a matriz $\Lambda$ possa ser escrita como $\Lambda=R^{2}$, temos pela unicidade da solução da equação diferencial que 


$$
\varphi(a)=(1 \cdot R) \tanh (R a)
$$

No entanto isso é imediato, já que $\Lambda$ é por definição uma matriz positiva semidefinida (temos que $v \cdot \Lambda v:=\left\langle(v \cdot X)^{2}\right\rangle \geq 0, v \in \mathbb{R}^{d}$, em que definimos a esperança $\langle\cdot\rangle:=\mathbb{E}[\cdot \cosh (a \cdot X)] / \mathbb{E}[\cosh (a \cdot X)])$. Assim sendo, $\Lambda$ é também o quadrado de outra matriz positiva semi-definida (mais que isso, $\Lambda$ e $R$ são matrizes reais simétricas (vide [16])).

Agora, para $\left\{a_{\alpha}\right\}$ e $\left\{Y_{\alpha}\right\} \in \mathbb{R}_{+}, 1 \cdot Y \tanh (a \cdot Y)$ é uma função monótona crescente de $\left\{Y_{\alpha}\right\}$. O lema estará provado se verificarmos a desigualdade

$$
(1 \cdot \Lambda) 1-(1 \cdot \bar{\Lambda}) 1=\frac{\mathbb{E}\left[(1 \cdot X)^{2} \cosh (a \cdot X)\right]}{\mathbb{E}[\cosh (a \cdot X)]}-(1 \cdot \bar{\Lambda}) 1 \geq 0
$$

para qualquer $a=\gamma 1>0, \gamma>0$.

Explicitamente:

$$
(1 \cdot \bar{\Lambda}) 1=\left(1 \cdot \bar{R}^{2}\right) 1=\sum_{\alpha}\left(\bar{R}^{2} 1\right)_{\alpha}=\sum_{\alpha} \bar{\lambda}_{\alpha}^{2}=\sum_{\alpha} \mathbb{E}\left[X_{\alpha}^{2}\right]
$$

Segue da simetria da distribuição de probabilidade conjunta do vetor $X=X_{\alpha}$ (produtos $X_{\alpha} X_{\beta}$ exibem sempre alguma variável de spin elevada a uma potência ímpar) que

$$
\mathbb{E}\left[X_{\alpha} X_{\beta}\right]=\delta_{\alpha \beta} \mathbb{E}\left[X_{\alpha}^{2}\right]
$$

e portanto

$$
\mathbb{E}\left[\left(\sum_{\alpha} X_{\alpha}\right)^{2}\right]=\sum_{\alpha, \beta} \mathbb{E}\left[X_{\alpha} X_{\beta}\right]=\sum_{\alpha} \mathbb{E}\left[X_{\alpha}^{2}\right]
$$

Desse modo, 


$$
(1 \cdot \bar{\Lambda}) 1=\mathbb{E}\left[(1 \cdot X)^{2}\right]
$$

e assim, a condição (A.2) pode ser escrita como

$$
(1 \cdot \Lambda) 1-(1 \cdot \bar{\Lambda}) 1=\frac{\mathbb{E}\left[(1 \cdot X)^{2} \cosh (a \cdot X)\right]}{\mathbb{E}[\cosh (a \cdot X)]}-\mathbb{E}\left[(1 \cdot X)^{2}\right] \geq 0
$$

para todo $a$. A relação (A.3) é verdadeira, uma vez que esta pode ser reescrita como $(1 \cdot \Lambda) 1-(1 \cdot \bar{\Lambda}) 1=\frac{1}{2} \frac{\mathbb{E}\left[\left\{(1 \cdot X)^{2}-(1 \cdot Y)^{2}\right\}\{\cosh (a \cdot X)-\cosh (a \cdot Y)\}\right]}{\mathbb{E}[\cosh (a \cdot X)]} \geq 0$

com $X$ e $Y$ dois vetores aleatórios independentes e igualmente distribuídos. Isso conclui a prova do lema. 


\section{Apêndice B}

\section{Dominação gaussiana ,}

\section{positividade por reflexão e}

\section{estimativa do tabuleiro de xadrez}

A fim de descrever o conceito de positividade por reflexão OS (ou de OsterwalderSchrader), tomemos uma cadeia de Ising de $2 n$ spins nos sítios $\pm 1 / 2, \pm 3 / 2, \ldots$, $\pm(n-1 / 2)$.

Seja

$$
\langle\cdot\rangle_{0}=\frac{1}{2^{2 n}} \sum_{\left\{\sigma_{ \pm(j-1 / 2)}\right\} \in\{-1,1\}} . \quad, \quad j=1, \ldots, n
$$

o valor esperado livre; para uma função $F$ dos spins $\sigma_{i}, i>0$, seja $\theta F$ a mesma função dos spins $\sigma_{-i}$, ou seja, se $F=f\left(\sigma_{1 / 2}, \sigma_{3 / 2}, \ldots\right)$ então vale a identidade $\theta F=f\left(\sigma_{-1 / 2}, \sigma_{-3 / 2}, \ldots\right)(f$ real $)$. A positividade OS para $\langle\cdot\rangle_{0}$ é a desigualdade

$$
\begin{aligned}
\langle(\theta F) F\rangle_{0} & =\frac{1}{2^{4 n}} \sum_{\sigma_{i}, \sigma_{-i} \in\{-1,1\}} f\left(\left\{\sigma_{-i}\right\}\right) f\left(\left\{\sigma_{i}\right\}\right) \\
& =\frac{1}{2^{2 n}} \sum_{\sigma_{-i} \in\{-1,1\}} f\left(\left\{\sigma_{-i}\right\}\right) \quad \frac{1}{2^{2 n}} \sum_{\sigma_{i} \in\{-1,1\}} f\left(\left\{\sigma_{i}\right\}\right) \\
& =\langle\theta F\rangle_{0}\langle F\rangle_{0} \\
& =\langle F\rangle_{0}^{2} \geq 0 .
\end{aligned}
$$


Dado um hamiltoniano $H(\sigma)$ definimos, como de hábito, a média térmica

$$
\langle\cdot\rangle=\frac{\left\langle\cdot e^{-\beta H}\right\rangle_{0}}{\left\langle e^{-\beta H}\right\rangle_{0}}
$$

Note que o ferromagneto com vizinhos mais próximos (o último sítio da rede interage com o primeiro)

$$
-H=\sum_{i=-n+1 / 2}^{n-3 / 2} \sigma_{i} \sigma_{i+1}+\sigma_{(-n+1 / 2)} \sigma_{(n-1 / 2)}
$$

é da forma

$$
-H=A+\theta A+C_{1} \theta C_{1}+C_{2} \theta C_{2},
$$

com

$$
A=\sum_{i=1 / 2}^{n-3 / 2} \sigma_{i} \sigma_{i+1}, \quad C_{1}=\sigma_{1 / 2}, \quad C_{2}=\sigma_{(n-1 / 2)}
$$

Teorema B.1 Se $-H=A+\theta A+\sum_{i=1}^{k} C_{i} \theta C_{i}$ e se $\langle\cdot\rangle_{0}$ é positivo por reflexão (PR), então $<\cdot>$, definido em (B.1), é PR.

Prova. Como $\theta$ é um morfismo (morfismo significa $\theta(A B)=\theta(A) \theta(B)$ ) temos que

$$
e^{-\beta H}=e^{-\beta A} \theta\left(e^{-\beta A}\right) e^{-\beta \sum C_{l} \theta C_{l}} .
$$

Expandindo a exponencial, vemos que

$$
e^{-\beta H}=\text { soma de termos da forma }\left(D_{1} \theta D_{1} \cdots D_{j} \theta D_{j}\right),
$$


e assim da positividade por reflexão de $\langle\cdot\rangle_{0}$ concluímos que $\left\langle e^{-\beta H}\right\rangle_{0} \geq 0$ e que $\left\langle e^{-\beta H} A_{1} \theta A_{1} \cdots A_{m} \theta A_{m}\right\rangle_{0} \geq 0$, ou seja, $\left\langle A_{1} \theta A_{1} \cdots A_{m} \theta A_{m}\right\rangle \geq 0$.

De (B.2) e do Teorema (B.1) obtemos

$$
\langle(\theta F) F\rangle \geq 0
$$

a positividade por reflexão para o modelo interagente.

Observe que com condições periódicas podemos obter positividade por reflexão para uma grande variedade de planos involvendo reflexões sobre as diversas formas de se dividir o círculo em duas metades. Ainda mais, (B.3) leva a uma desigualdade de Schwarz, que podemos iterar usando estes diferentes planos de reflexão.

Por exemplo, considere uma cadeia de quatro spins, $\sigma_{1}, \sigma_{2}, \sigma_{3}, \sigma_{4}$, acoplados pelo hamiltoniano $-H=\beta\left(\sigma_{1} \sigma_{2}+\sigma_{2} \sigma_{3}+\sigma_{3} \sigma_{4}+\sigma_{4} \sigma_{1}\right)$. Sejam $f_{1}, \ldots, f_{4}$ quatro funções a valores reais em \pm 1 . Então

$$
\begin{aligned}
\left\langle f_{1}\left(\sigma_{1}\right) f_{2}\left(\sigma_{2}\right) f_{3}\left(\sigma_{3}\right) f_{4}\left(\sigma_{4}\right)\right\rangle \leq & \left\langle f_{1}\left(\sigma_{1}\right) f_{1}\left(\sigma_{4}\right) f_{2}\left(\sigma_{2}\right) f_{2}\left(\sigma_{3}\right)\right\rangle^{1 / 2} \times \\
& \times\left\langle f_{4}\left(\sigma_{1}\right) f_{4}\left(\sigma_{4}\right) f_{3}\left(\sigma_{2}\right) f_{3}\left(\sigma_{3}\right)\right\rangle^{1 / 2} \\
\leq & \prod_{i=1}^{4}\left\langle f_{i}\left(\sigma_{1}\right) \cdots f_{i}\left(\sigma_{4}\right)\right\rangle^{1 / 4} .
\end{aligned}
$$

Na primeira etapa usamos a desigualdade de Schwarz para as reflexões $\theta \sigma_{1}=\sigma_{4}$ e $\theta \sigma_{2}=\sigma_{3}$ : definimos $F\left(\sigma_{1}, \sigma_{2}\right)=f_{1}\left(\sigma_{1}\right) f_{2}\left(\sigma_{2}\right)$ e $G\left(\sigma_{1}, \sigma_{2}\right)=f_{4}\left(\sigma_{1}\right) f_{3}\left(\sigma_{2}\right)$, de modo que

$$
\left\langle f_{1}\left(\sigma_{1}\right) f_{2}\left(\sigma_{2}\right) f_{3}\left(\sigma_{3}\right) f_{4}\left(\sigma_{4}\right)\right\rangle=\langle F(\theta G)\rangle \leq\langle F(\theta F)\rangle^{1 / 2}\langle G(\theta G)\rangle^{1 / 2} .
$$

Em seguida, usamos a desigualdade correspondente às reflexões $\theta^{\prime} \sigma_{1}=\sigma_{2} \mathrm{e}$ $\theta^{\prime} \sigma_{4}=\sigma_{3}$ : definimos $F_{i}\left(\sigma_{1}, \sigma_{4}\right)=f_{i}\left(\sigma_{1}\right) f_{i}\left(\sigma_{4}\right), i=1, \ldots, 4$, de modo que

$$
\begin{aligned}
\langle F(\theta F)\rangle^{1 / 2}\langle G(\theta G)\rangle^{1 / 2} & =\left\langle F_{1}\left(\theta^{\prime} F_{2}\right)\right\rangle^{1 / 2}\left\langle F_{4}\left(\theta^{\prime} F_{3}\right)\right\rangle^{1 / 2} \\
& \leq \prod_{i=1}^{4}\left\langle F_{i}\left(\theta^{\prime} F_{i}\right)\right\rangle^{1 / 4}=\prod_{i=1}^{4}\left\langle f_{i}\left(\sigma_{1}\right) \cdots f_{i}\left(\sigma_{4}\right)\right\rangle^{1 / 4}
\end{aligned}
$$


Repetindo esse argumento e aplicando-o a planos de reflexão envolvendo todas as direções possíveis, obtemos

Proposição B.2 (Estimativas de tabuleiro de xadrez para o modelo $N$-vetorial)

$$
\left\langle\prod_{x \in \Lambda} f_{x}(\sigma(x))\right\rangle \leq \prod_{x \in \Lambda}\left\langle\prod_{y \in \Lambda} f_{x}(\sigma(y))\right\rangle^{1 /|\Lambda|}
$$

em que definimos a média térmica de uma função $f_{x}$ de $\mathbb{R}^{N}$ como sendo

$$
<\cdot>=Z^{-1} \int \cdot e^{-\beta H} \prod_{x \in \Lambda} d \mu(\sigma(x))
$$

com $H$ definido por (5.1.3).

Proposição B.3 A dominação gaussiana $Z(\eta(x)) \leq Z(0)$ é válida para o modelo $N$-vetorial.

Prova. É suficiente provar a relação (5.1.11) para o caso $d \mu(\sigma)=F(\sigma) d \sigma$, com $F(\sigma) \neq 0$ para todo $\sigma$, e então usar um argumento limite e obter uma estimativa para $d \mu(\sigma)=\delta\left(|\sigma|^{2}-1\right) d \sigma$. Nesse caso, definamos $G_{x}(\sigma)=F(\sigma-\eta(x)) / F(\sigma)$ de modo que

$$
\begin{aligned}
Z(\eta) & =\int\left\{\prod_{x \in \Lambda} F(\sigma(x)) d \sigma(x)\right\} e^{-\beta H(\sigma+\eta)} \\
& =\int\left\{\prod_{x \in \Lambda} \frac{F(\sigma(x)-\eta(x))}{F(\sigma(x))} F(\sigma(x)) d \sigma(x)\right\} e^{-\beta H(\sigma)} \\
& =Z(0)\left\langle\prod_{x \in \Lambda} G_{x}(\sigma(x))\right\rangle \\
& \leq Z(0) \prod_{x \in \Lambda}\left\langle\prod_{y \in \Lambda} G_{x}(\sigma(y))\right\rangle^{1 /|\Lambda|}=Z(0)
\end{aligned}
$$

em que a desigualdade segue da estimativa do tabuleiro de xadrez e o último passo segue se notarmos que 


$$
\left\langle\prod_{y \in \Lambda} G_{x}(\sigma(y))\right\rangle=\frac{\int \prod_{y \in \Lambda} d \mu(\sigma(y)-\eta(x)) e^{-\beta H(\sigma)}}{\int \prod_{y \in \Lambda} d \mu(\sigma(y)) e^{-\beta H(\sigma)}}=1
$$

uma vez que $\mathrm{H}$ é apenas função de $\sigma(x)-\sigma(y)=(\sigma(x)-\eta(x))-(\sigma(y)-\eta(x))$. Com isso concluímos a prova da proposição.

Fica claro que o emprego da estimativa do tabuleiro de xadrez leva à "homogenização" da perturbação $\eta$, já que ao tomarmos a média sobre os spins, fixamos um mesmo $\eta(x)$ para todos os sítios. 


\section{Apêndice C}

\section{Positividade por reflexão para o laplaciano hierárquico}

Os resultados aqui apresentados, literalmente extraídos de [18], são uma extensão dos resultados do apêndice B. O conceito de positividade por reflexão e a desigualdade do tabuleiro de xadrez empregados são precisamente os mesmos.

Seja $P$ um plano perpendicular a algum eixo coordenado que divida $\Lambda$ em duas metades $\Lambda_{ \pm}$, disjuntas e complementares:

$$
\Lambda=\Lambda_{+} \cup \Lambda_{-} \quad \text { e } \quad \Lambda_{+} \cup \Lambda_{-}=\emptyset
$$

( $P$ corta perpendicularmente ligações, porém não intercepta sítios de $\Lambda$ ). Seja

$$
r: \Lambda_{+} \longrightarrow \Lambda_{-}
$$

um mapa que associa a cada $j \in \Lambda_{+}$sua imagem refletida $r j \in \Lambda_{-}$, isto é, o sítio simétrico com respeito a $P$. O mapa $r$ induz um morfismo linear $\pi_{P}: \mathcal{U}_{+} \rightarrow \mathcal{U}_{-}$, na álgebra abeliana $\mathcal{U}_{ \pm}$das funções limitadas no espaço de configuração $\Omega_{ \pm}=\mathbb{R}^{\Lambda_{ \pm}}$, expresso por

$$
\pi_{P} F\left(\left\{x_{j}\right\}_{j \in \Lambda_{+}}\right)=F\left(\left\{x_{r j}\right\}_{j \in \Lambda_{+}}\right)
$$

para todo $F \in \mathcal{U}_{+}$. 
Reescrevamos a definição de positividade por reflexão (apresentada anteriormente no apêndice B):

Definição C.1 (Positividade por Reflexão) Um estado $\langle\cdot\rangle_{\nu}$ é dito ser um funcional positivo por reflexão se

$$
\left\langle F \pi_{P}(F)\right\rangle_{\nu} \geq 0
$$

seguir para todo $F \in \mathcal{U}_{+}$.

O funcional $\langle\cdot\rangle_{\nu}$, definido como o valor esperado com respeito à medida $\nu$ (com $A=-\Delta \otimes I,-\Delta$ o laplaciano usual), com condições periódicas de contorno, é, como vimos, positivo por reflexão. Supondo a validade de (C.1), temos a desigualdade de Schwarz

$$
\left\langle F \pi_{P}(G)\right\rangle_{\nu} \leq\left\langle F \pi_{P}(F)\right\rangle_{\nu}^{1 / 2}\left\langle G \pi_{P}(G)\right\rangle_{\nu}^{1 / 2}
$$

Se aplicarmos (C.2) a todo plano $P$ que corta perpendicularmente as ligações entre os sítios, vemos que $\langle\cdot\rangle_{\nu}$ satisfaz a desigualdade do tabuleiro de xadrez

$$
\left\langle\prod_{j} F_{j}\left(h_{j}\right)\right\rangle_{\nu} \leq \prod_{j}\left\langle\prod_{i} F_{i}\left(h_{j}\right)\right\rangle_{\nu}^{1 / n}
$$

em que, para cada $j, F_{j}\left(h,\left\{x_{i}\right\}_{i \in \Lambda}\right)=F\left(h, x_{j}\right)$ é uma família uniparamétrica $h$ de funções limitadas em $\mathbb{R}^{N}$.

Temos, portanto, da positividade por reflexão do funcional $\langle\cdot\rangle_{\nu}$, a desigualdade do tabuleiro de xadrez, que nos permite provar o resultado de dominação gaussiana (via Proposição B.3). Provemos então a validade de (C.1).

Seja $\theta=\left(\theta_{1}, \ldots, \theta_{K}\right), \theta_{k} \in\{0,1\}$ a representação binária de um ponto $i \in\left\{0,1, \ldots, 2^{K}-1\right\}$ (por exemplo, o número 5 pode ser escrito nesta base como $\left.1 \cdot 2^{2}+1 \cdot 2^{0}=(1,0,1,0, \ldots, 0)\right)$. O mapa $r$ é expresso, com respeito ao plano de reflexão $P_{k}$ na $k$-ésima hierarquia, como 


$$
(r \theta)_{l}= \begin{cases}\theta_{l} & \text { se } \quad l \neq k \\ 1-\theta_{l} & \text { se } \quad l=k\end{cases}
$$

e age trocando cada par de blocos consecutivos de tamanho $2^{k-1}$ indexados por $\tau=\left(\theta_{k+1}, \ldots, \theta_{K}\right):\left\{i_{1}, \ldots, i_{2^{k-1}}, j_{1}, \ldots, j_{2^{k-1}}\right\} \rightarrow\left\{j_{1}, \ldots, j_{2^{k-1}}, i_{1}, \ldots, i_{2^{k-1}}\right\}$. Sejam, por exemplo, $K=4$ e $k=2$. A aplicação do mapa $r$ sobre o número $\theta=15=(1,1,1,1)$ resulta em $r \theta=(1,0,1,1)=13$, exatamente o número simétrico a 15 com respeito a um bloco de tamanho $2^{2-1}=2$.

Para uma rede $d$-dimensional, um ponto arbitrário $i$ pode ser representado por $\theta=\left(\theta_{1}, \ldots, \theta_{K}\right), \theta_{k}$ assumindo valores em uma caixa $B_{L}=\{0,1, \ldots, L-1\}^{d}$ com condições periódicas de contorno: $\theta_{k}=\left(\theta_{k, 1}, \ldots, \theta_{k, d}\right)$, com $\theta_{k, d} \in\{0,1, \ldots, L-1\}$ $\bmod L$. Como feito anteriormente, escolhemos um plano $P$ perpendicular aos eixos coordenados que intercepte ligações, e não sítios, dividindo $\{0,1, \ldots, L-1\}^{d}$ em duas metades disjuntas $B_{L}=B_{L}^{+} \cup B_{L}^{-}$. O mapa $r$, com respeito a um plano de reflexão $P$ na $k$-ésima hierarquia, associa a cada $\theta$ sua imagem $r \theta$, com $(r \theta)_{l}=\theta_{l}$ se $l \neq k$ e $(r \theta)_{k} \in B_{L}^{ \pm}$se $\theta_{k} \in B_{L}^{\mp}$.

Seja portanto

$$
\Lambda_{ \pm}=\left\{\theta: \theta_{k} \in B_{L}^{ \pm}\right\}
$$

a partição de $\Lambda_{K}=\left\{0, \ldots, L^{K}-1\right\}$ em duas metades com respeito ao plano $P$ na $k$-ésima hierarquia e seja $\mathcal{P}_{ \pm}$o conjunto de polinômios em $x_{\theta}, \theta \in \Lambda_{ \pm}$. O mapa, como vimos, induz um morfismo linear $\pi_{P}: \mathcal{P}_{+} \rightarrow \mathcal{P}_{-}$, dado por

$$
\pi_{P} F\left(\left\{x_{\theta}\right\}_{\theta \in \Lambda_{+}}\right)=F\left(\left\{x_{r \theta}\right\}_{\theta \in \Lambda_{+}}\right)
$$

Para provarmos a positividade por reflexão da medida hierárquica do modelo $\mathrm{N}$-vetorial, basta mostrarmos que $J$, definido por (5.2.36), é uma interação positiva por reflexão. Para tanto, seja $y=B^{l} x \in \mathbb{R}^{\Lambda_{K-l}}$ e note que $y_{\tau} \in \mathcal{P}_{ \pm}$, caso $\theta_{k} \in B_{L}^{ \pm}$ seja uma coordenada de $\tau=\left(\theta_{l+1}, \ldots, \theta_{K}\right)$ para $l<k$ (a variável de bloco $y$ contém somente elementos de uma das classes). Caso $l \geq k, y$ pode ser decomposto como 


$$
y=y^{+}+y^{-},
$$

com $y^{ \pm} \in \mathcal{P}_{ \pm}$e tal que $y^{-}=\pi_{P} y^{+}$. Temos para $l<k$

$$
\begin{aligned}
(y, y)_{\Lambda_{K-l}} & =\sum_{\tau: \theta_{k} \in B_{L}^{+}}\left|y_{\tau}\right|^{2}+\sum_{\tau: \theta_{k} \in B_{L}^{-}}\left|y_{\tau}\right|^{2} \\
& =\sum_{\tau: \theta_{k} \in B_{L}^{+}}\left|y_{\tau}\right|^{2}+\sum_{\tau: \theta_{k} \in B_{L}^{+}}\left|\pi_{P} y_{\tau}\right|^{2} \\
& =\sum_{\tau: \theta_{k} \in B_{L}^{+}}\left|y_{\tau}\right|^{2}+\pi_{P} \sum_{\tau: \theta_{k} \in B_{L}^{+}}\left|y_{\tau}\right|^{2} \equiv\|y\|_{+}^{2}+\pi_{P}\|y\|_{+}^{2} \geq 0
\end{aligned}
$$

pela propriedade do morfismo $\left(\sum_{i} \pi_{P}\left(F_{i}\right)=\pi_{P} \sum_{i} F_{i}\right)$. Para $l \geq k$

$$
\begin{aligned}
(y, y)_{\Lambda_{K-l}} & =\sum_{\tau}\left|y_{\tau}^{+}+y_{\tau}^{-}\right|^{2} \\
& =\sum_{\tau}\left|y_{\tau}^{+}\right|^{2}+\sum_{\tau}\left|y_{\tau}^{-}\right|^{2}+2 \sum_{\tau} y_{\tau}^{+} y_{\tau}^{-} \\
& \left.\equiv\left\|\left.y\right|_{+} ^{2}+\pi_{P}\right\| y\right|_{+} ^{2}+2 \sum_{\tau} y_{\tau}^{+} \pi_{P} y_{\tau}^{+} \geq 0 .
\end{aligned}
$$

Os resultados acima, juntamente com (5.2.36), implicam

$$
\begin{aligned}
(x, J x)_{\Lambda_{K}} & =\sum_{k=1}^{K} L^{-2 k}\left(x,\left(B^{*}\right)^{k} B^{k} x\right)_{\Lambda_{K}} \\
& =\sum_{k=1}^{K} L^{-2 k}\left(B^{k} x, B^{k} x\right)_{\Lambda_{K-k}} \\
& =\sum_{k=1}^{K} L^{-2 k}(y, y)_{\Lambda_{K-k}} \geq 0,
\end{aligned}
$$

isto é, $J$ é uma interação positiva por reflexão. Daí segue a positividade por reflexão da medida $\nu$, e assim a do funcional $\langle\cdot\rangle_{\nu}$. 


\section{Referências Bibliográficas}

[1] Aizenman, M., Wehr J., Comm. Math. Phys. 130, 489 (1990).

[2] Bricmont, J., Kupiainen, A., Comm. Math. Phys. 116, 539 (1988).

[3] Bricmont, J., Kupiainen, A., J. Stat. Phys. 51, 1021 (1988).

[4] Dyson, F., J., Comm. Math. Phys. 12, 91 (1969).

[5] Dyson, F., J., Comm. Math. Phys. 21, 269 (1971).

[6] Fisher, D.S., Fröhlich, J., Spencer T., J. Stat. Phys. 34, 871 (1984).

[7] Fortuin, C.M., Kasteleyn, P.W., Ginibre, J., Commun. Math. Phys. 22, 89 (1971).

[8] Fröhlich, J., Imbrie, J.Z., Comm. Math. Phys. 96, 145 (1984).

[9] Fröhlich, J., Simon, B., Spencer T., Comm. Math. Phys. 50, 79 (1976).

[10] Fröhlich, J., Spencer T., Comm. Math. Phys. 84, 79 (1982).

[11] Griffiths, R., "Rigorous Results and Theorems", em Phase Transitions (C. Domb, M.S. Green ed.), Vol. I, cap. 2, Academic Press Inc. (London) Ltd. (1972).

[12] Griffiths, R., Phys. Rev. A 136, 437 (1964).

[13] Imbrie, J.Z., Comm. Math. Phys. 98, 145 (1985).

[14] Imry, Y., Ma, S.-K., Phys. Rev. Lett. 35, 1399 (1975). 
[15] Khorunzhy, A.M, Khoruzhenko, B.A., Pastur, L.A., Shecherbina, M.V., "The Large- $n$ Limit in Statistical Mechanics and the Spectral Theory of Disordered Systems", em Phase Transitions Vol. 13, Domb e Lebowitz ed. (1992).

[16] Lancaster, P., Tismenetsky, M., "The Theory of Matrices". San Diego: Academic Press, 1985 (segunda edição).

[17] Marchetti, D.H.U., "Renormalization Group in Statistical Mechanics: Ideas, Techniques and Analyses", Notas de aula não publicadas, IFUSP(1996).

[18] Conti, W.R.P., Marchetti, D.H.U., "Hierarchical Spherical Model as a Viscosity Limit of Corresponding $O(N)$ Heisenberg Model", Preprint (2006), artigo gentilmente cedido pelos autores.

[19] Perez, J.F., Rev. Bras. Fis. 10, 296 (1980).

[20] Perez, J.F., Wreszinki, W.F., van Hemmen, J.L., J. Stat. Phys. 35, 89 (1984).

[21] Pirogov, S.A., Sinai, Ya.G., Theor. and Math. Phys. 25, 1185 (1975); 26, 39 (1976).

[22] Pontin, L.F., Baêta Segundo, J.A., Perez, J.F., "Random Field Ising Model without Internal Contours: Phase Transition in Dimensional $d \geq 2$ ". Preprint (1995).

[23] Simon, B., Ann. Israel Phys. Soc. 2, 287 (1978). 